\title{
AN AXISYMMETRIC BOUNDARY LAYER ON A NEEDLE
}

\author{
A. D. BRYUNO AND T. V. SHADRINA
}

\begin{abstract}
Methods of power geometry are used to study the boundary layer on a semi-infinite needle, due to a steady flow of a viscous fluid or gas parallel to the needle. The purpose is to find the asymptotics of the flow in the boundary layer at infinity along the needle. Two variants of the flow are considered: (a) an incompressible nonheat-conducting fluid, and (b) a compressible heat-conducting gas. It is shown that variant (a) has no asymptotics for solutions satisfying all the boundary conditions, whereas variant (b) has several families of asymptotics for solutions that satisfy all the boundary conditions. These asymptotic expansions have power or logarithmic singularities near the needle.
\end{abstract}

\section{INTRODUCTION}

Approximately 100 years ago Prandtl [1] and Blasius [2] did the original work on the theory of boundary layers on a semi-infinite plate in a steady flow of a viscous incompressible fluid. Subsequently it turned out that the Blasius solution can also be applied to thick plates with a rounded leading edge, to sharpened plates, and to a finite plate (except for both its edges). Goldstein [3] (1933) considered a flow behind a plate; later these results were refined by Stewartson [4] (1957). In 1970, van de Vooren and Dijkstra [5 studied the boundary layer on the whole length of a plate, including near the leading edge. MacLachlan [6] (1991) constructed a mathematical model of a flow past a thin finite plate, in which the boundary layer has three layers.

The boundary layer for an axisymmetric flow past a cylinder has also been studied in many papers. At the initial part of the cylinder, where the thickness of the layer is small compared to the radius, the influence of the transverse curvature can be neglected. Here the boundary layer is not any different from the boundary layer on a plate and can be described by the Blasius solution. The nearer to the nose of the cylinder, the less accurate is the approximation given by the Blasius solution. Seban and Bond 7] (1951) and a bit later Kelly [8] (1954) obtained a solution that extends the Blasius solution as it approaches the nose of the cylinder. To study the boundary layer as it moves away from the origin of the cylinder, first Lord Rayleigh's method [9] (1911) was used, which yielded a rough approximation. The solutions obtained by this method gave a qualitative description of the boundary layer, but not a quantitative one. Pohlhausen [10] (1921) proposed a method, using which Glauert and Lighthill 11 (1955) gave an approximate solution of the problem of a flow past a long thin cylinder, which is valid for any values of the quantity $\nu x /\left(u_{\infty} a^{2}\right)$, where $\nu$ is the dynamical coefficient of viscosity, $u_{\infty}$ is the speed of the outer flow, $a$ is the radius of the cylinder, and $x$ is an independent variable directed

2000 Mathematics Subject Classification. Primary 76D10, 76N20; Secondary 35B40, 34E05, 35A25, 35C20, 35Q30, 35Q35, 76D05, 80A20.

Key words and phrases. Boundary layer, asymptotics, Navier-Stokes equations, power geometry, incompressible viscous fluid, compressible gas.

This research was carried out with the support of the Russian Foundation for Basic Research (grant 05-01-00050).

(C)2007 American Mathematical Society 
along the cylinder. In addition, they also found an asymptotic solution corresponding to large values of this parameter. At the same time, Stewartson [12] studied the more general case of the boundary layer on a long thin cylinder where the speed of the outer flow is given by a power function $u_{\infty}=c x^{m}$.

However, the results obtained for the cylinder do not give a limit as the radius of the cylinder tends to zero. So until now there has been no theory for the boundary layer on a semi-infinite needle.

Power geometry, which is used in the present paper, was developed by Bryuno as a universal set of algorithms for the analysis of singularities that is suitable for all types of equations. It can deal with algebraic equations, ordinary or partial differential equations; systems can consist of equations of the same type or contain equations of different types. In [13, Ch. VI, §6] power geometry was used for studying a steady flow of a viscous incompressible fluid past a semi-infinite plate, and the Blasius solution was obtained. In addition, for the first time a purely mathematical justification was given for the theory of a boundary layer on a plate, without recourse to any mechanical or physical considerations.

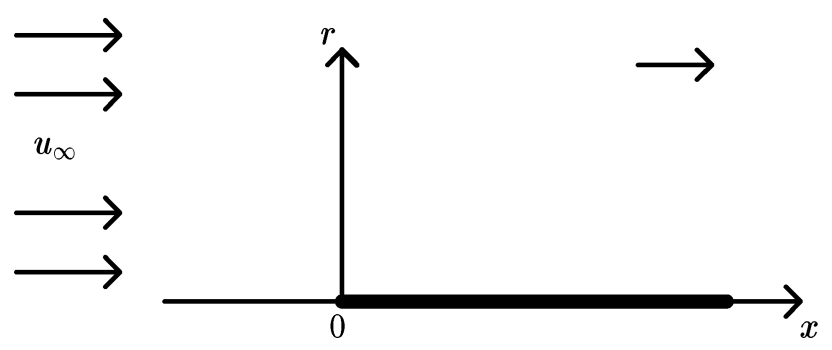

Figure 1. Scheme of an axisymmetric flow past a needle.

In this paper we consider a steady axisymmetric flow of a viscous fluid or gas running straight at a semi-infinite needle (Figure 1) for two cases: (a) an incompressible fluid, and (b) a compressible heat-conducting gas. Such a flow is described by the Navier-Stokes equations, which reduce to a system of partial differential equations for two independent variables: $x$, along the symmetry axis, and $r$, the distance from the $x$-axis. In variant (a) the dependent variables are the stream function $\psi$ and pressure $p$. In the case of a viscous compressible heat-conducting gas flowing past a needle, one more dependent variable is added. Instead of the pressure $p$, two dependent variables are used: the enthalpy $h$ (an analogue of temperature) and the density $\rho$. In both cases the needle is given as $x \geq 0$, $r=0$.

The purpose of this paper is to find the asymptotics, as $x \rightarrow+\infty$, of solutions for which the dependent variables satisfy all the boundary conditions (if such solutions exist).

To do this we use the methods of power geometry. Using the techniques of spatial power geometry we extract from the complete system a truncated system which is a first approximation to the complete system as $x \rightarrow+\infty$. Furthermore, the solutions of this truncated system satisfy the boundary conditions at infinity. Next, using the methods of planar power geometry we analyze the resulting truncated system, which in a number of cases reduces to a single equation. In the case of a viscous compressible heat-conducting gas flowing past the needle, after the asymptotic behaviour of solutions near the needle and at the outer boundary of the boundary layer are obtained, solutions of the truncated system are computed numerically by the Runge-Kutta method.

The paper contains three chapters. In Chapter I we describe the notions and methods of power geometry, which are used in Chapters II and III. Spatial power geometry, which 
is described in $\S 1$ of Chapter I, allows us to select and simplify a truncated system of equations whose solutions give strong asymptotics for solutions of the original system. Planar power geometry, whose notions and methods are expounded in $\S 2$ of Chapter I, allows us to obtain not only the asymptotic behaviour of solutions but also asymptotic expansions of solutions. In a number of cases these expansions converge and give the solutions themselves.

In Chapter II we investigate the boundary layer for an axisymmetric flow of a viscous incompressible fluid past a semi-infinite needle. In $\S 1$ we show that such a flow can be described by a system of two partial differential equations for the stream function $\psi$ and pressure $p$ with two independent variables: $x$ along the axis of symmetry, and the distance $r$ from the $x$-axis. The needle is given as $x \geq 0, r=0$. The boundary conditions are given at infinity as

$$
\psi=\frac{u_{\infty} r^{2}}{2}, \quad p=p_{0} \quad \text { as } x \rightarrow-\infty, \quad \text { where } u_{\infty}, p_{0}=\text { const } \neq 0,
$$

which can be replaced by

$$
\psi=r^{2}, \quad p=p_{0} \quad \text { as } r \rightarrow+\infty, \quad \text { where } p_{0}=\text { const } \neq 0,
$$

and at the needle (the adhesion condition) as

$$
\frac{\partial \psi}{\partial x}=\frac{\partial \psi}{\partial r}=\frac{\partial^{2} \psi}{\partial x \partial r}=\frac{\partial^{2} \psi}{\partial r^{2}}=0 \quad \text { for } x \geq 0, r=0 .
$$

In $\S 2$, using the methods of spatial power geometry expounded in $\S 1$ of Chapter I, we select a truncated system of equations that describes the flow near the needle as $x \rightarrow+\infty$. After the introduction of the self-similar variables

$$
\xi=\frac{r^{2}}{x}, \quad h(\xi)=\frac{\psi}{x}, \quad p(\xi)=p,
$$

the truncated system becomes a system of two ordinary differential equations, which reduces to a single third-order ordinary differential equation for $h(\xi)$. In $\S 3$, the asymptotic analysis of its solutions by the methods of planar power geometry, detailed in $\S 2$ of Chapter I, shows that this equation has no solutions satisfying the adhesion boundary conditions at the needle (2). In $\S 4$ of Chapter II we prove that the resulting truncated system corresponding to the boundary layer near the needle as $x \rightarrow+\infty$ also has no non-self-similar solutions satisfying the boundary condition (2). To do this we make the change of variables

$$
x=x, \quad \xi=\frac{r^{2}}{x}, \quad h(x, \xi)=\frac{\psi}{x}, \quad p(x, \xi)=p ;
$$

that is, we take $x$ and $\xi$ for the independent variables. The resulting system reduces to a single partial differential equation for $h(x, \xi)$, which involves $x$ only in the form of $\ln x$. As $\ln x \rightarrow+\infty$, a first approximation of this equation is given by the equation that coincides exactly with the ordinary differential equation obtained in the self-similar coordinates. In spite of the fact that in this case $h$ depends on $\ln x$ too, the solutions of the resulting equation still do not satisfy the adhesion boundary conditions at the needle.

In $\S \S 5$ and 6 of Chapter II we consider the possibility of the existence of a two-layer solution of the original system satisfying the boundary conditions (1) and (2). To do this, in $\S 5$ we use the methods of spatial power geometry to extract a truncated system that describes the flow of the fluid in the layer that immediately adjoins the layer near the needle from the original system. After introducing the self-similar coordinates

$$
\eta=\frac{r^{2}}{x^{2}}, \quad g(\eta)=\frac{\psi}{x^{2}}, \quad p(\eta)=p,
$$


this truncated system becomes a system of two ordinary differential equations, which reduces to a single second-order equation for $g(\eta)$. Asymptotic analysis of the solutions of the latter equation by methods of planar power geometry shows that this equation has solutions which have asymptotics of two types as $\eta \rightarrow 0$ :

$$
\begin{aligned}
& \text { a) } \quad g \sim \text { const, } \quad p \sim-\frac{a}{\eta}, \quad a=\text { const }>0, \\
& \text { b) } g=\eta, \quad p=p_{0}=\text { const. }
\end{aligned}
$$

Consequently, in case a) the pressure $p$ tends to $-\infty$ as $\eta \rightarrow 0$, which has no physical meaning. In case b) we have

$$
\psi=r^{2}, \quad p=p_{0}=\text { const }
$$

on the entire outer layer; that is, we obtain the one-layer variant considered in $\S 3$.

Next, in $\S 6$ of Chapter II we consider the possibility of the existence of a two-layer non-self-similar solution. For this, similarly to the case of a one-layer solution, we make the change of variables

$$
x=x, \quad \eta=\frac{r^{2}}{x^{2}}, \quad g(x, \eta)=\frac{\psi}{x^{2}}, \quad p(x, \eta)=p
$$

in the truncated system corresponding to the outer layer. The resulting system involves $x$ only in the form of $\ln x$. A first approximation as $\ln x \rightarrow+\infty$ of the system obtained is given by the system that coincides exactly with the system of ordinary differential equations obtained on the outer layer after introduction of the self-similar coordinates (4); that is, as $\eta \rightarrow 0$ there are two types of asymptotic behaviour for the solution:

$$
\begin{aligned}
& \text { a) } \quad g \sim \text { const, } \quad p \sim-\frac{a}{\eta}, \quad a=\text { const }>0, \\
& \text { b) } g \sim \eta, \quad p \sim p_{0}=\text { const. }
\end{aligned}
$$

Consequently, in case a) the pressure $p$ tends to $-\infty$ as $\eta \rightarrow+0$, which has no physical meaning. In case $\mathrm{b}), p \rightarrow$ const and we obtain the boundary condition

$$
\psi \sim r^{2}, \quad p \sim \text { const }
$$

at the outer boundary of the inner layer. From the viewpoint of spatial power geometry, when the truncated systems are selected describing the flow in the inner layer, the variant of the boundary conditions (6) is similar to the variant of the boundary conditions (5). Consequently, in case (6) the truncated system describing the flow in the inner layer coincides with the system for the one-layer solution, whose non-self-similar solutions are considered in $\S 4$ of Chapter II and which has no solutions satisfying the boundary condition (2).

The main results of Chapter II are the theorems in which we prove that for the problem of a steady axisymmetric flow of a viscous incompressible fluid past a semi-infinite needle as $x \rightarrow+\infty$ there are no solutions satisfying all the boundary conditions (1), (2).

In Chapter III we consider a problem with a larger number of dependent variables. This is the problem of a steady axisymmetric flow of a viscous compressible heat-conducting gas past a semi-infinite needle. Such a flow is described by a system of three partial differential equations for the stream function $\psi$, density $\rho$, and enthalpy $h$ (an analogue of temperature) with two independent variables: $x$ (along the symmetry axis) and $r$ (the distance from the $x$-axis). As in Chapter II, the needle is given by $x \geq 0$, $r=0$. The boundary conditions are given at infinity as

(7) $\psi=\psi_{0} r^{2}, \quad \rho=\rho_{0}, \quad h=h_{0} \quad$ at $x=-\infty, \quad$ where $\psi_{0}, \rho_{0}, h_{0}=$ const $\neq 0$,

and at the needle by $(2)$. In $\S 1$, the methods of spatial power geometry are used to select a truncated system that describes the flow in the boundary layer near the needle 
as $x \rightarrow+\infty$. It turns out that $\rho h=$ const for its self-similar solutions. Therefore, after introducing the self-similar coordinates

$$
\xi=\frac{r^{2}}{x}, \quad G(\xi)=\frac{\psi}{x}, \quad \mathrm{P}(\xi)=\rho, \quad H(\xi)=h,
$$

the truncated system reduces to a system of two ordinary differential equations for $G(\xi)$ and $H(\xi)$. In $\S 2$, we distinguish the invariant manifold $G^{\prime} H=1$ for this system, where it reduces to a single ordinary second-order differential equation for $H(\xi)$.

In $\S \S 3-5$, the asymptotic analysis of its solutions by methods of planar power geometry is used to show that this equation has solutions that satisfy the boundary conditions at the needle and at infinity: as $\xi \rightarrow 0$ they have asymptotic behaviour

$$
\begin{gathered}
H \sim \text { const } \xi^{\lambda}, \quad \lambda<0, \quad \text { for } n=0 \\
\text { (that is, } \left.\quad \psi \sim \operatorname{const} x \xi^{1-\lambda}, \quad \rho \sim \text { const } \xi^{1-\lambda}\right), \\
H \sim \text { const }|\ln \xi|^{1 / n} \quad \text { for } n \in(0,1] \\
\text { (that is, } \left.\quad \psi \sim \text { const } \frac{r^{2}}{|\ln \xi|^{1 / n}}, \quad \rho \sim \text { const }|\ln \xi|^{-1 / n}\right),
\end{gathered}
$$

and as $\xi \rightarrow+\infty$,

$$
H-1 \sim \text { const } \int \xi^{s} e^{-\xi / 2} d \xi,
$$

where the constant $n \in[0,1]$ is the exponent in the power law $\mu / \mu_{0}=\left(T / T_{0}\right)^{n}$ giving the connection between the dynamical coefficient of viscosity $\mu$ and the absolute temperature $T$. Solutions with the asymptotics (9)-(11) are found theoretically.

In $\S 6$ of Chapter III we describe a numerical method, which is used to find the dependencies between the constants in (9)-(11) for $n=0,1 / 4,1 / 2,3 / 4,1$. The results of the computations are given in Tables 36 .

In $\S 7$ we return to the original problem and state the main result of Chapter III, namely that the problem of axisymmetric flow of a viscous compressible heat-conducting gas past a semi-infinite needle in the boundary layer as $x \rightarrow+\infty$ has families of solutions which have asymptotic behaviour given by (9), (10) near the needle.

The Conclusion reflects the discussion provoked by this paper.

The results obtained in Chapters II and III were announced in [14-23]. The preprints [25, 24] give the first version of a detailed exposition; the preprints [26, 27, 28] give the second detailed version, which is vastly different from the first. This is the third detailed exposition, which is vastly different from both of the previous ones.

We have numbered the sections, lemmas, theorems, corollaries, remarks, and formulae separately in each chapter. The first number in a formula's label is the number of a section. Tables and figures are numbered consecutively throughout the paper.

The authors are grateful to V.A. Kondrat'ev and M. M. Vasil'ev for useful remarks.

\section{CHAPTER I. ELEMENTS OF POWER GEOMETRY}

In this chapter we give a brief exposition of some of the notions and results from power geometry, which are used in Chapters II and III. Spatial power geometry allows us to select and simplify a truncated system of equations whose solutions give strong asymptotics for solutions of the original system. Planar power geometry allows us to obtain not only asymptotics of solutions but also asymptotic expansions of solutions. In a number of cases these expansions converge and give the solutions themselves. 


\section{Spatial POWER GeOMETRY}

Here we briefly explain some notions of power geometry [13], which are used in $\S 2$ of Chapter II and in $\S \S 1$ and 7 of Chapter III. New results are given with brief proofs.

We denote by $X^{\prime}=\left(x_{1}, \ldots, x_{l}\right)$ the vector of independent variables, and by $X^{\prime \prime}=$ $\left(x_{l+1}, \ldots, x_{l+m}\right)$ the vector of dependent variables. We set $n=l+m$ and combine all the variables into one vector $X=\left(X^{\prime}, X^{\prime \prime}\right)=\left(x_{1}, \ldots, x_{n}\right) \in \mathbb{R}^{n}$. A differential monomial $a(X)$ is by definition the product of an ordinary monomial

$$
c X^{R} \stackrel{\text { def }}{=} c x_{1}^{r_{1}} \cdots x_{n}^{r_{n}},
$$

where $c=$ const $\in \mathbb{R}$ and $R=\left(r_{1}, \ldots, r_{n}\right) \in \mathbb{R}^{n}$, and finitely many partial derivatives of the form

$$
\frac{\partial^{k} x_{j}}{\partial x_{1}^{k_{1}} \partial x_{2}^{k_{2}} \cdots \partial x_{l}^{k_{l}}} \stackrel{\text { def }}{=} \frac{\partial^{\left\|K^{\prime}\right\|} x_{j}}{\partial X^{\prime K^{\prime}}}, \quad l<j \leq n,
$$

where $k=\left|k_{1}\right|+\cdots+\left|k_{l}\right| \stackrel{\text { def }}{=} \| K^{\prime}||$ for $K^{\prime}=\left(k_{1}, \ldots, k_{l}\right)$. With each differential monomial $a(X)$ we associate its vectorial exponent $Q(a) \in \mathbb{R}^{n}$ by the following rule:

$$
Q\left(c X^{R}\right)=R ; \quad Q\left(\frac{\partial^{\left\|K^{\prime}\right\|} x_{j}}{\partial X^{\prime K^{\prime}}}\right)=\left(-K^{\prime}, E_{j}\right),
$$

where $j>l$ and $E_{j}$ denotes the $j$-th unit vector; when two monomials are multiplied, their vectorial exponents are added: $Q\left(a_{1} a_{2}\right)=Q\left(a_{1}\right)+Q\left(a_{2}\right)$, where $a_{1}$ and $a_{2}$ are differential monomials.

A finite sum of differential monomials

$$
f(X)=\sum a_{k}(X)
$$

is called a differential sum. With this sum we associate the set in $\mathbb{R}^{n}$ consisting of the vectorial exponents of its monomials $\mathbf{S}(f)=\left\{Q\left(a_{k}\right)\right\}$, called the support of the sum (1.1). The convex hull $\Gamma(f)$ of the support $\mathbf{S}(f)$ is called the polyhedron of the sum (1.1). Its boundary $\partial \Gamma(f)$ consists of faces $\Gamma_{j}^{(d)}$, where $d=\operatorname{dim}\left(\Gamma_{j}^{(d)}\right)$ is the dimension of the face, and $j$ its number.

Let $\mathbb{R}_{*}^{n}$ denote the dual space of the space $\mathbb{R}^{n}$, so that the scalar product

$$
\langle P, Q\rangle \stackrel{\text { def }}{=} p_{1} q_{1}+\cdots+p_{n} q_{n}
$$

is defined for $P=\left(p_{1}, \ldots, p_{n}\right) \in \mathbb{R}_{*}^{n}$ and $Q=\left(q_{1}, \ldots, q_{n}\right) \in \mathbb{R}^{n}$.

On a family of curves

$$
x_{i}=b_{i} \tau^{p_{i}}, \quad b_{i} \neq 0, \quad p_{i} \in \mathbb{R}, \quad \tau \rightarrow \infty, \quad i=1, \ldots, n,
$$

where the $b_{i}$ are any constants in $\mathbb{R}$, a differential monomial $a$ behaves as const $\tau^{\langle P, Q\rangle}$, where $Q=Q(a), P=\left(p_{1}, \ldots, p_{n}\right) \in \mathbb{R}_{*}^{n}$. Therefore on this family of curves the leading monomials of the sum $f(X)$ are those for which the scalar product $\langle P, Q\rangle$ is the greatest over the points of the support of the sum $f(X)$, that is, $\langle P, Q\rangle=\max$ over $Q \in \mathbf{S}(f)$. To find these maximum points we consider the convex hull $\Gamma(f)$ of the support $\mathbf{S}(f)$, that is, the polyhedron of the sum (1.1). To each vector $P \neq 0, P \in \mathbb{R}_{*}^{n}$, there is a corresponding support face $\Gamma_{j}^{(d)}$ such that

$$
\begin{aligned}
& \left\langle P, Q_{1}\right\rangle=\left\langle P, Q_{2}\right\rangle, \quad Q_{1}, Q_{2} \in \Gamma_{j}^{(d)}, \\
& \left\langle P, Q_{1}\right\rangle>\langle P, Q\rangle, \quad Q \in \mathbf{S}(f) \backslash \Gamma_{j}^{(d)} .
\end{aligned}
$$

The vector $P$ is an exterior normal vector to the face $\Gamma_{j}^{(d)}$; that is, it is directed from this face outside the polyhedron $\Gamma(f)$. The set $\mathbf{U}_{j}^{(d)}$ of all vectors $P$ with a fixed support 
face $\Gamma_{j}^{(d)}$ is called the normal cone of the face $\Gamma_{j}^{(d)}$ and is described by the system of equalities and inequalities (1.3); that is, the set $\mathbf{U}_{j}^{(d)}$ is described by the formula

$$
\mathbf{U}_{j}^{(d)}=\left\{P:\left\langle P, Q_{1}\right\rangle=\left\langle P, Q_{2}\right\rangle, Q_{1}, Q_{2} \in \Gamma_{j}^{(d)} ;\left\langle P, Q_{1}\right\rangle>\langle P, Q\rangle, Q \in \mathbf{S} \backslash \Gamma_{j}^{(d)}\right\} .
$$

To each face $\Gamma_{j}^{(d)}$ there corresponds the truncated sum

$$
\hat{f}_{j}^{(d)}(X)=\sum a_{k}(X) \quad \text { over } k \text { such that } Q\left(a_{k}\right) \in \Gamma_{j}^{(d)} .
$$

According to [13, Ch. VI] each truncated sum $\widehat{f}_{j}^{(d)}(X)$ is a first approximation of the sum $f(X)$ as the vector $\ln |X|=\left(\ln \left|x_{1}\right|, \ldots, \ln \left|x_{n}\right|\right)$ tends to infinity near the normal cone $\mathbf{U}_{j}^{(d)}$. In particular, a motion $\tau \rightarrow \infty$ along a curve of the family (1.2) corresponds to the vector $\ln |X|=\left(\ln \left|x_{1}\right|, \ldots, \ln \left|x_{n}\right|\right)$ tending to infinity along the ray $\lambda P$ as $\lambda \rightarrow+\infty$; that is, all the non-zero components of the vector $\ln |X|$ tend to infinity.

The extended normal cone $\check{\mathbf{U}}_{j}^{(d)}$ of a face $\Gamma_{j}^{(d)}$ is defined to be the union of the normal cone $\mathbf{U}_{j}^{(d)}$ and the normal cones of the faces contained in the face $\Gamma_{j}^{(d)}$. The meaning of this notion is that the truncation $\widehat{f}_{j}^{(d)}(X)$ corresponding to the face $\Gamma_{j}^{(d)}$ contains all the summands that are leading when the vector $\ln |X|$ tends to $\infty$ near the extended normal cone $\check{\mathbf{U}}_{j}^{(d)}$.

We consider a system of differential equations

$$
f_{i}(X)=0, \quad i=1, \ldots, m,
$$

where the $f_{i}(X)$ are differential sums. Corresponding to each sum are its support $\mathbf{S}\left(f_{i}\right)$, the polyhedron $\Gamma\left(f_{i}\right)$, the set of faces $\Gamma_{i j_{i}}^{\left(d_{i}\right)}$, and the set of truncated equations $\widehat{f}_{i j_{i}}^{\left(d_{i}\right)}(X)$ $=0$. Suppose the support faces $\Gamma_{i j_{i}}^{\left(d_{i}\right)}, i=1, \ldots, m$, correspond to a vector $P \neq 0$. Then to $P$ there corresponds the truncated system of equations

$$
\widehat{f}_{i j_{i}}^{\left(d_{i}\right)}(X)=0, \quad i=1, \ldots, m .
$$

According to [13, Ch. III, $\S 1]$, the order of a function $\varphi(\tau)$ as $\tau \rightarrow \infty$ is

$$
p(\varphi) \stackrel{\text { def }}{=} \lim \frac{\ln |\varphi(\tau)|}{\ln \tau}
$$

if the limit exists. Functions $\varphi(\tau)$ and $\psi(\tau)$ are strongly asymptotically equivalent if

$$
\frac{\varphi(\tau)-\psi(\tau)}{\varphi(\tau)}=O\left(\tau^{-\varepsilon}\right)
$$

as $\tau \rightarrow \infty$, where $\varepsilon>0$.

Vector functions $\Phi(X)=\left(\varphi_{1}(X), \ldots, \varphi_{m}(X)\right)$ and $\Psi(X)=\left(\psi_{1}(X), \ldots, \psi_{m}(X)\right)$ are strongly asymptotically equivalent on the family of curves (1.2) if on this family

$$
\frac{\varphi_{j}(X)-\psi_{j}(X)}{\varphi_{j}(X)}=O\left(\tau^{-\varepsilon}\right), \quad j=1, \ldots, m,
$$

as $\tau \rightarrow \infty$, where $\varepsilon>0$.

We now wish to state and prove an assertion of the following type.

Assertion. Suppose that the system of equations (1.4) has a solution

$$
x_{j}=\varphi_{j}\left(X^{\prime}\right), \quad j=l+1, \ldots, n,
$$

which is defined on the family (1.2); that is, on this family all the $\varphi_{j}\left(X^{\prime}\right)$ have certain orders $p_{j}$ when

$$
x_{i}=b_{i} \tau^{p_{i}}, \quad i=1, \ldots, l .
$$


Then the truncated system (1.5) corresponding to the vector $P=\left(p_{1}, \ldots, p_{n}\right)$ has a solution

$$
x_{j}=\psi_{j}\left(X^{\prime}\right), \quad j=l+1, \ldots, n,
$$

that is strongly asymptotically equivalent to the solution (1.7) on the family (1.8).

For the case where all the $\varphi_{j}\left(X^{\prime}\right)$ in (1.7) expand into series in powers of $X^{\prime}$, this assertion follows from Theorem 1.1 of Chapter VI in [13]. However, this is not sufficient for our purposes. Therefore, below we state and briefly prove a more general version of this assertion, which is what we shall be using.

Definitions. In items 1-4 we consider functions $\varphi(\tau)$ that are defined for $\tau>\tau_{0}=$ const.

1. We denote by $\mathcal{R}(p)$ the set of functions $\varphi(\tau)$ such that

$$
\limsup _{\tau \rightarrow \infty} \frac{\ln |\varphi(\tau)|}{\ln \tau} \leq p .
$$

2. We denote by $\mathcal{P}(0)$ a class of functions $\varphi(\tau)$ with order $p(\varphi) \leq 0$ that is closed under multiplication by a real constant, taking a real power, addition and multiplication, and has the following property: if the order satisfies $p(\varphi)<0$, then $\varphi(\tau) \equiv 0$.

For example, a class $\mathcal{P}(0)$ may be generated from $\varphi(\tau) \equiv 1$ and $\varphi(\tau)=\ln \tau$ by the operations indicated in the definition, as well as by taking logarithms and converging sums of asymptotic series.

3. We denote by $\mathcal{P}(p)$ the class of functions $\varphi(\tau)$ of the form $\varphi(\tau)=\psi(\tau) \tau^{p}$, where $\psi(\tau) \in \mathcal{P}(0)$.

4. We denote by $\mathcal{P}^{*}(p)$ the class of functions $\varphi(\tau)$ of the form $\varphi(\tau)=\widehat{\varphi}(\tau)+\widetilde{\varphi}(\tau)$, where $\widehat{\varphi}(\tau) \in \mathcal{P}(p)$ and $\widetilde{\varphi}(\tau) \in \mathcal{R}(p-\varepsilon)$ for $\varepsilon>0$.

5. We denote by $\mathcal{P}\left(P^{\prime}, p\right)$ the class of functions $\varphi\left(X^{\prime}\right)$ that are functions of class $\mathcal{P}(p)$ on the family (1.8) and all the partial derivatives satisfy

$$
\frac{\partial^{k} \varphi\left(X^{\prime}\right)}{\partial X^{\prime K^{\prime}}} \in \mathcal{P}\left(p-\left\langle P^{\prime}, K^{\prime}\right\rangle\right),
$$

where $P^{\prime}=\left(p_{1}, \ldots, p_{l}\right)$.

This means that at each single differentiation of the function $\varphi\left(X^{\prime}\right)$ the order decreases by one. Consequently, functions of the type $\sin x, \cos x$ are excluded.

6. We denote by $\mathcal{R}\left(P^{\prime}, p\right)$ the class of functions $\varphi\left(X^{\prime}\right)$ for which property (1.10) holds on the family (1.8) and for all the derivatives $\partial^{k} \varphi\left(X^{\prime}\right) / \partial X^{\prime K^{\prime}}$, the upper limit analogous to (1.10) is $\leq p-\left\langle P^{\prime}, K^{\prime}\right\rangle$.

7. We denote by $\mathcal{P}^{*}\left(P^{\prime}, p\right)$ the class of functions $\varphi\left(X^{\prime}\right)$ of the form $\varphi\left(X^{\prime}\right)=\widehat{\varphi}\left(X^{\prime}\right)+$ $\widetilde{\varphi}\left(X^{\prime}\right)$, where $\widehat{\varphi}\left(X^{\prime}\right) \in \mathcal{P}\left(P^{\prime}, p\right)$ and $\widetilde{\varphi}\left(X^{\prime}\right) \in \mathcal{R}\left(P^{\prime}, p-\varepsilon\right)$ for $\varepsilon>0$.

8. We denote by $\mathcal{P}\left(P^{\prime}, P^{\prime \prime}\right)$ the class of vector functions

$$
\Phi^{\prime \prime}\left(X^{\prime}\right)=\left(\varphi_{l+1}\left(X^{\prime}\right), \ldots, \varphi_{n}\left(X^{\prime}\right)\right)
$$

such that

$$
\varphi_{j}\left(X^{\prime}\right) \in \mathcal{P}\left(P^{\prime}, p_{j}\right), \quad j=l+1, \ldots, n,
$$

where $P^{\prime \prime}=\left(p_{l+1}, \ldots, p_{n}\right)$ for the $p_{j}$ in (1.11).

9 . We denote by $\mathcal{P}^{*}\left(P^{\prime}, P^{\prime \prime}\right)$ the class of vector functions

$$
\Phi^{\prime \prime}\left(X^{\prime}\right)=\left(\varphi_{l+1}\left(X^{\prime}\right), \ldots, \varphi_{n}\left(X^{\prime}\right)\right)
$$

that have the form

$$
\Phi^{\prime \prime}\left(X^{\prime}\right)=\widehat{\Phi}^{\prime \prime}\left(X^{\prime}\right)+\widetilde{\Phi}^{\prime \prime}\left(X^{\prime}\right)
$$


where

$$
\widehat{\Phi}^{\prime \prime}\left(X^{\prime}\right) \in \mathcal{P}\left(P^{\prime}, P^{\prime \prime}\right)
$$

and

$$
\widetilde{\Phi}^{\prime \prime}\left(X^{\prime}\right)=\left(\widetilde{\varphi}_{l+1}\left(X^{\prime}\right), \ldots, \widetilde{\varphi}_{n}\left(X^{\prime}\right)\right)
$$

for

$$
\widetilde{\varphi}_{j}\left(X^{\prime}\right) \in \mathcal{R}\left(P^{\prime}, p_{j}-\varepsilon\right), \quad j=l+1, \ldots, n, \quad \varepsilon>0 .
$$

Theorem 1. Suppose that the system of equations (1.4) has a solution (1.7) that belongs to the class $\mathcal{P}^{*}\left(P^{\prime}, P^{\prime \prime}\right)$. Then the truncated system (1.5) corresponding to the vector $P=\left(P^{\prime}, P^{\prime \prime}\right)$ has a solution

$$
X^{\prime \prime}=\widehat{\Phi}^{\prime \prime}\left(X^{\prime}\right) .
$$

Proof. First we state two auxiliary properties a) and b), which follow immediately from the definitions (throughout, $\varepsilon>0$ ).

a) Let $a\left(X^{\prime}, X^{\prime \prime}\right)$ be a differential monomial, and $Q(a)=Q$ its vectorial exponent.

If $\Phi^{\prime \prime}\left(X^{\prime}\right) \in \mathcal{P}\left(P^{\prime}, P^{\prime \prime}\right)$ and $P=\left(P^{\prime}, P^{\prime \prime}\right)$, then $a\left(X^{\prime}, \Phi^{\prime \prime}\left(X^{\prime}\right)\right) \in \mathcal{P}(\langle P, Q\rangle)$.

If $\Phi^{\prime \prime}\left(X^{\prime}\right) \in \mathcal{P}^{*}\left(P^{\prime}, P^{\prime \prime}\right)$, then

$$
a\left(X^{\prime}, \Phi^{\prime \prime}\left(X^{\prime}\right)\right)=a\left(X^{\prime}, \widehat{\Phi}^{\prime \prime}\left(X^{\prime}\right)\right)+b\left(X^{\prime}\right) \in \mathcal{P}^{*}\left(P^{\prime},\langle P, Q\rangle\right),
$$

where $a\left(X^{\prime}, \widehat{\Phi}^{\prime \prime}\left(X^{\prime}\right)\right) \in \mathcal{P}\left(P^{\prime},\langle P, Q\rangle\right)$ and $b\left(X^{\prime}\right) \in \mathcal{R}\left(P^{\prime},\langle P, Q\rangle-\varepsilon\right)$.

b) Let $f\left(X^{\prime}, X^{\prime \prime}\right)$ be a differential sum, and $\widehat{f}\left(X^{\prime}, X^{\prime \prime}\right)$ its truncated sum corresponding to a vector $P=\left(P^{\prime}, P^{\prime \prime}\right)$ and containing a monomial $a\left(X^{\prime}, X^{\prime \prime}\right)$ with vectorial exponent $Q(a)=Q$.

If $\Phi^{\prime \prime}\left(X^{\prime}\right) \in \mathcal{P}\left(P^{\prime}, P^{\prime \prime}\right)$, then

$$
f\left(X^{\prime}, \Phi^{\prime \prime}\left(X^{\prime}\right)\right)=\widehat{f}\left(X^{\prime}, \widehat{\Phi}^{\prime \prime}\left(X^{\prime}\right)\right)+g\left(X^{\prime}\right) \in \mathcal{P}^{*}\left(P^{\prime},\langle P, Q\rangle\right),
$$

where $\widehat{f}\left(X^{\prime}, \widehat{\Phi}^{\prime \prime}\left(X^{\prime}\right)\right) \in \mathcal{P}\left(P^{\prime},\langle P, Q\rangle\right)$ and $g\left(X^{\prime}\right) \in \mathcal{R}\left(P^{\prime},\langle P, Q\rangle-\varepsilon\right)$.

We now move on to the proof of the theorem. If $X^{\prime \prime}=\Phi^{\prime \prime}\left(X^{\prime}\right)$ is a solution of (1.4), then each of the equations (1.4) turns into the identity

$$
f_{i}\left(X^{\prime}, \Phi^{\prime \prime}\left(X^{\prime}\right)\right) \equiv 0 .
$$

Suppose that $\widehat{f}_{i}(X)$ contains a monomial $a_{i}(X)$ with exponent $Q\left(a_{i}\right)=Q_{i}$. According to property b) we have

$$
f_{i}\left(X^{\prime}, \Phi^{\prime \prime}\left(X^{\prime}\right)\right)=\widehat{f_{i}}\left(X^{\prime}, \widehat{\Phi}^{\prime \prime}\left(X^{\prime}\right)\right)+h_{i}\left(X^{\prime}\right) \in \mathcal{P}^{*}\left(P^{\prime},\left\langle P, Q_{i}\right\rangle\right),
$$

where

$$
\widehat{f_{i}}\left(X^{\prime}, \widehat{\Phi}^{\prime \prime}\left(X^{\prime}\right)\right)=g_{i}\left(B^{\prime}, \tau\right) \tau^{\left\langle P, Q_{i}\right\rangle} \in \mathcal{P}\left(\left\langle P, Q_{i}\right\rangle\right)
$$

on the family (1.8) and $h_{i} \in \mathcal{R}(\langle P, Q\rangle-\varepsilon)$. Consequently, $g_{i}\left(B^{\prime}, \tau\right) \equiv 0$. But this means that

$$
\widehat{f}_{i}\left(X^{\prime}, \Phi^{\prime \prime}\left(X^{\prime}\right)\right)=0 .
$$

Since this is valid for each $i=1, \ldots, m$, the truncated system (1.5) has the solution (1.12).

Example. The truncated system (1.5) is quasi-homogeneous; that is, it is transformed into itself under the change of variables

$$
x_{i}=\mu^{p_{i}} \widetilde{x}_{i}, \quad i=1, \ldots, n,
$$

where $\mu \in \mathbb{R}$. According to [29, the solution (1.12) of (1.5) is also quasi-homogeneous (or self-similar) if it is transformed into itself under the change of variables (1.15). We claim that under a quasi-homogeneous substitution in (1.12), the function $g_{i}\left(B^{\prime}, \tau\right)$ in 
the expression (1.14) is independent of $\tau$. Indeed, make the change of variables (1.15) in (1.13). Then using (1.15) followed by (1.14) we obtain the two equalities

$$
\widehat{f}_{i}\left(\mu^{P^{\prime}} \widetilde{X}^{\prime}, \mu^{P^{\prime \prime}} \widetilde{\Phi^{\prime \prime}}\left(\mu^{P^{\prime}} \widetilde{X}^{\prime}\right)\right)=\mu^{\left\langle P, Q_{i}\right\rangle} \widehat{f}_{i}\left(\widetilde{X}^{\prime}, \widehat{\Phi}^{\prime \prime}\left(\widetilde{X}^{\prime}\right)\right)=g_{i}\left(B^{\prime}, \mu\right) \mu^{\left\langle P, Q_{i}\right\rangle} .
$$

But here $\mu=\tau$ and $\widetilde{X}^{\prime}=B^{\prime}$. Consequently, $g_{i}\left(B^{\prime}, \tau\right) \equiv \widehat{f}_{i}\left(B^{\prime}, \widehat{\Phi}^{\prime \prime}\left(B^{\prime}\right)\right)$; that is, it is independent of $\tau$. Therefore, when the solutions (1.7) of the system (1.4) with self-similar first approximations (1.12) are considered, it is sufficient to take $\mathcal{P}(0)$ to be the set of constants, that is, $\mathcal{P}(0)=\mathbb{R}$.

This means that Theorem 1 is valid for all those solutions whose first approximation is self-similar and the additions are regular under differentiation.

Remark 1. Using Theorem 3.3 in [30] we can estimate the quantity $\varepsilon$ in formula (1.6), that is, we can estimate the accuracy of the asymptotics given by formula (1.12) according to (1.9). If for $\tau$ we take one of the independent variables $x_{k}$ that tends to infinity, $0<k \leq l$, then the corresponding coordinate of the vector $P$ is $p_{k}=1$. Then

$$
0<\varepsilon<\varepsilon_{0}=\min _{1 \leq i \leq m}\left|\alpha_{i}-\beta_{i}\right|,
$$

where

$$
\alpha_{i}=\langle P, Q\rangle, \quad Q \in \Gamma_{i j_{i}}^{\left(d_{i}\right)}, \quad \beta_{i}=\left\langle P, Q_{1}\right\rangle, \quad Q_{1} \in \mathbf{S}\left(f_{i}\right) \backslash \Gamma_{i j_{i}}^{\left(d_{i}\right)},
$$

the vector $P$ is as in Theorem 1 , and the $\Gamma_{i j_{i}}^{\left(d_{i}\right)}$ are the faces corresponding to the equations of the truncated system $(1.5), i=1, \ldots, m$.

Consequently, by selecting truncated systems and solving them we can obtain the asymptotic behaviour of solutions of the original system. The supports of the equations of the truncated system are situated on the support planes with a common normal vector $P$; that is, the truncated systems are quasi-homogeneous and have self-similar solutions 29]. In self-similar variables, each truncated system reduces to a system with fewer independent variables (see Theorem 3 below).

The normal cone of the truncated system (1.5) is defined to be the set

$$
\mathbf{U}_{J}^{(D)}=\mathbf{U}_{1 j_{1}}^{\left(d_{1}\right)} \cap \mathbf{U}_{2 j_{2}}^{\left(d_{2}\right)} \cap \cdots \cap \mathbf{U}_{m j_{m}}^{\left(d_{m}\right)},
$$

where $D=\left(d_{1}, \ldots, d_{m}\right)$ and $J=\left(j_{1}, \ldots, j_{m}\right)$, that is, this is the intersection of the normal cones of all the truncated equations involved in the truncated system. The extended normal cone of the truncated system (1.5) is defined to be the intersection of the extended normal cones of all the truncated equations involved in the truncated system:

$$
\check{\mathbf{U}}_{J}^{(D)}=\check{\mathbf{U}}_{1 j_{1}}^{\left(d_{1}\right)} \cap \check{\mathbf{U}}_{2 j_{2}}^{\left(d_{2}\right)} \cap \cdots \cap \check{\mathbf{U}}_{m j_{m}}^{\left(d_{m}\right)},
$$

where $D=\left(d_{1}, \ldots, d_{m}\right)$ and $J=\left(j_{1}, \ldots, j_{m}\right)$.

Suppose that, apart from the system of equations (1.4), the problem has boundary conditions of the form

$$
\begin{gathered}
f_{j} \stackrel{\text { def }}{=} x_{j+l-m}-c_{j} X^{\prime R_{j}^{\prime}}=0, \quad R_{j}^{\prime}=\left(r_{1 j}, \ldots, r_{l j}\right), \quad c_{j}=\text { const } \neq 0, \\
\text { as } x_{k} \rightarrow \infty, \quad j=m+1, \ldots, m+m^{\prime}, \quad m^{\prime} \leq m .
\end{gathered}
$$

These equations can be regarded as additional to the system (1.4). To them there correspond vectorial exponents and all the other elements of power geometry. We are faced with the problem of selecting a truncated system such that its solution satisfies the boundary conditions (1.16). One can apply the technique of selecting a truncation to the equations in (1.16). But they are themselves quasi-homogeneous; therefore the only meaningful truncations coincide with the equations themselves. This is possible only for 
certain values of the vector $P$, those that guarantee that, for each of the equations (1.16), the truncation coincides with the equation itself. By using these conditions on the vector $P$ one can reduce the dimension of the space of exponents $Q$ and consider the problem of finding truncations in this space of lower dimension.

The vector $P=\left(p_{1}, \ldots, p_{n}\right)=\left(P^{\prime}, P^{\prime \prime}\right)$ must satisfy the conditions

$$
\left\langle P, Q_{1}\right\rangle=\left\langle P, Q_{2}\right\rangle, \quad Q_{1}, Q_{2} \in \mathbf{S}\left(f_{j}\right), \quad j=m+1, \ldots, m+m^{\prime} .
$$

The system of equations $(1.17)$ can be resolved and $p_{l+1}, \ldots, p_{l+m^{\prime}}$ can be expressed explicitly in terms of $P^{\prime}=\left(p_{1}, \ldots, p_{l}\right)$, that is,

$$
p_{j+l-m}=\left\langle P^{\prime}, R_{j}^{\prime}\right\rangle, \quad R_{j}^{\prime}=\left(r_{1 j}, \ldots, r_{l j}\right), \quad j=m+1, \ldots, m+m^{\prime} .
$$

According to these conditions, the supports of the equations of the system (1.4) can be projected onto a space of lower dimension. Then, in the spaces $\mathbb{R}_{*}^{n}$ and $\mathbb{R}^{n}$ of vectors $P$ and $Q$, we obtain projections $P \rightarrow \widetilde{P}$ and $Q \rightarrow \widetilde{Q}$ by the following rule:

$$
\langle P, Q\rangle=\langle\widetilde{P}, \widetilde{Q}\rangle
$$

for the vectors

$$
\begin{aligned}
& \widetilde{P}=\left(p_{1}, \ldots, p_{l}, p_{l+m^{\prime}+1}, \ldots, p_{n}\right), \\
& \widetilde{Q}=\left(\widetilde{q}_{1}, \ldots, \widetilde{q}_{l}, q_{l+m^{\prime}+1}, \ldots, q_{n}\right), \\
& \widetilde{q}_{i}=q_{i}+\sum_{j=m+1}^{m+m^{\prime}} q_{j+l-m} r_{i j}, \quad i=1, \ldots, l .
\end{aligned}
$$

Theorem 2. The projections $Q \rightarrow \widetilde{Q}$ and $P \rightarrow \widetilde{P}$, given by the rules (1.19) and (1.20) in the spaces $\mathbb{R}^{n}$ and $\mathbb{R}_{*}^{n}$ respectively, correspond to the boundary conditions (1.16).

Proof. The equalities (1.18) correspond to the boundary conditions (1.16). A straightforward verification shows that (1.20) and (1.18) imply (1.19).

Normal cones in the space $\mathbb{R}_{*}^{n-m^{\prime}}$ correspond to the projection thus obtained with the points $\widetilde{Q} \in \mathbb{R}^{n-m^{\prime}}$.

Apart from boundary conditions of the form (1.16), usually there are also additional boundary conditions in the form of the vanishing of functions and derivatives on certain subsets of the space $X^{\prime}$. Certain sets of vectors $P$ or $\widetilde{P}$ in the space $\mathbb{R}_{*}^{n}$ or in its projection $\mathbb{R}_{*}^{n-m^{\prime}}$ correspond to these conditions. We are interested in those truncated systems whose extended normal cone includes these sets. Corresponding to the limiting process $x_{i} \rightarrow 0$ we have $p_{i} \leq 0 ; p_{i} \geq 0$ corresponds to $x_{i} \rightarrow \infty$. This must be used in the description of additional boundary conditions.

We are interested in the asymptotics of the solution of the system (1.4) as $x_{j} \rightarrow \infty$ (or $x_{j} \rightarrow 0$ ); corresponding to this $p_{j} \geq 0$ (or $\left.p_{j} \leq 0\right), j=1, \ldots, l$, in the space $\mathbb{R}_{*}^{n}$. Based on these conditions we find truncated systems of the form (1.5), whose extended normal cones contain the sets corresponding to the additional boundary conditions described above, and have the property that the coordinate $p_{i}$ is positive or negative, depending on whether the coordinate $x_{i}$ tends to infinity or zero. To each of these truncated systems there corresponds its own vector $P$ that is normal to the supports of the truncated equations and the supports of the boundary conditions (1.16).

Theorem 3 ([29, $\S 10])$. Suppose that we have the truncated system (1.5) and boundary conditions of the form (1.16) with vector $P=\left(P^{\prime}, P^{\prime \prime}\right)=\left(p_{1}, \ldots, p_{n}\right)$ that is normal to the supports of all these equations, that is, condition (1.18) is satisfied. Let $B_{1}^{\prime}, \ldots, B_{l-1}^{\prime}$ 
be a basis of the linear subspace of vectors $Q^{\prime}$ satisfying the equation $\left\langle P^{\prime}, Q^{\prime}\right\rangle=0$. Then the system of equations (1.5), (1.16) has self-similar coordinates $\xi_{i}, y_{j}$ :

$$
\begin{aligned}
\xi_{i}=X^{\prime B_{i}^{\prime}}, & & i=1, \ldots, l-1, \\
x_{j}=y_{j} X^{\prime T_{j}^{\prime}}, & & j=l+1, \ldots, n,
\end{aligned}
$$

where $\left\langle P^{\prime}, T_{j}^{\prime}\right\rangle=p_{j}$, in which it reduces to a system of $m$ equations for $m$ dependent variables $y_{l+1}, \ldots, y_{n}$ with independent variables $\xi_{1}, \ldots, \xi_{l-1}$. For $l=2$ this is a system of ordinary differential equations, and for $l=1$ of algebraic equations.

This method allows us to choose the truncated systems (1.5) and to find their self-similar solutions that satisfy given boundary conditions and give the asymptotic behaviour of solutions of the original system (1.4).

\section{Planar POWER GeOMetry}

2.1. Statement of the problem: Finding the power asymptotics for solutions $([31,32$, and [33, $\S 1])$. Suppose that we are given an ordinary differential equation

$$
f(X)=0,
$$

where $f(X)$ is a differential sum, $X=(x, y), \quad x$ is an independent variable, and the dependent variable $y$ occurs in $f(X)$ in integer powers. We set

$$
\omega=\left\{\begin{aligned}
-1 & \text { if } x \rightarrow 0 \\
1 & \text { if } x \rightarrow \infty
\end{aligned}\right.
$$

Suppose that $x$ tends to zero or to infinity, and equation (2.1) has a solution of the form

$$
y=c_{r} x^{r}+o\left(|x|^{r+\varepsilon}\right),
$$

where $c_{r}=$ const $\in \mathbb{R}, c_{r} \neq 0$, and the exponents satisfy $r, \varepsilon \in \mathbb{R}$ and $\varepsilon \omega<0$. Then the expression

$$
y=c_{r} x^{r}, \quad c_{r} \neq 0
$$

is a power asymptotic of the solution (2.3).

Problem 1. For the solutions $y=\varphi(x)$ of equation (2.1) find all the power asymptotics (2.4) as $x \rightarrow 0$ and as $x \rightarrow \infty$.

We repeat some of the definitions of $\S 1$ as applied to planar power geometry. With each differential monomial $a(X)$ we associate its (vectorial) exponent $Q(a)=\left(q_{1}, q_{2}\right) \in \mathbb{R}^{2}$ by the following rules. For a monomial we set $Q\left(c x^{r_{1}} y^{r_{2}}\right)=\left(r_{1}, r_{2}\right)$; for a derivative, $Q\left(d^{l} y / d x^{l}\right)=(-l, 1)$; when differential monomials are multiplied their exponents are added as vectors: $Q\left(a_{1} a_{2}\right)=Q\left(a_{1}\right)+Q\left(a_{2}\right)$. The set $\mathbf{S}(f)$ of the exponents $Q\left(a_{i}\right)$ of all the differential monomials $a_{i}(X)$ involved in a differential sum $f(X) \stackrel{\text { def }}{=} \sum a_{i}(X)$ is called the support of the sum. Clearly, $\mathbf{S}(f) \subseteq \mathbb{R}^{2}$. We denote by $f_{Q}(X)$ the sum of those monomials $a_{i}(X)$ in $f(X)$ for which $Q\left(a_{i}\right)=Q$. Then the differential sum can be written in the form

$$
f(X)=\sum f_{Q}(X) \quad \text { over } \quad Q \in \mathbf{S}(f) .
$$

The convex hull $\Gamma(f)$ of the support $\mathbf{S}(f)$ is called the polygon of the sum $f(X)$. The boundary $\partial \Gamma(f)$ of the polygon $\Gamma(f)$ consists of vertices $\Gamma_{j}^{(0)}$ and edges $\Gamma_{j}^{(1)}$, which are called (generalized) faces $\Gamma_{j}^{(d)}$, where the upper index indicates the dimension of the face, 
and the lower indicates its number. To each face $\Gamma_{j}^{(d)}$ there corresponds the truncated sum

$$
\widehat{f}_{j}^{(d)}(X)=\sum a_{i}(X) \quad \text { over } \quad Q\left(a_{i}\right) \in \mathbf{S}(f) \cap \Gamma_{j}^{(d)} \stackrel{\text { def }}{=} \mathbf{S}_{j}^{(d)} .
$$

Suppose that $\mathbb{R}_{*}^{2}$ is the dual plane of the plane $\mathbb{R}^{2}$, so that the scalar product

$$
\langle P, Q\rangle \stackrel{\text { def }}{=} p_{1} q_{1}+p_{2} q_{2}
$$

is defined for $P=\left(p_{1}, p_{2}\right) \in \mathbb{R}_{*}^{2}$ and $Q=\left(q_{1}, q_{2}\right) \in \mathbb{R}^{2}$. To each face $\Gamma_{j}^{(d)}$ there also corresponds in $\mathbb{R}_{*}^{2}$ its own normal cone

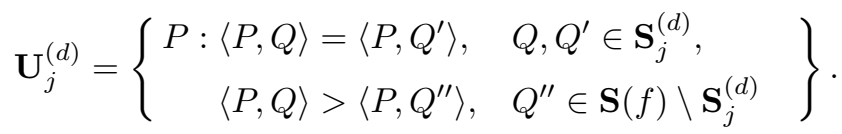

For an edge $\Gamma_{j}^{(1)}$, the normal cone $\mathbf{U}_{j}^{(d)}$ is the ray of the straight line orthogonal to the edge $\Gamma_{j}^{(1)}$ and passing through the origin $P=0$ that is directed from the edge $\Gamma_{j}^{(1)}$ outward from the polygon $\Gamma(f)$. For a vertex $\Gamma_{j}^{(0)}$, the normal cone $\mathbf{U}_{j}^{(0)}$ is the open sector (angle) on the plane $\mathbb{R}_{*}^{2}$ with vertex at the origin $P=0$ bounded by the rays that are the normal cones of the edges adjacent to the vertex $\Gamma_{j}^{(0)}$.

Thus, to each face $\Gamma_{j}^{(d)}$ of the support $\mathbf{S}(f)$ of equation (2.1) there correspond the normal cone $\mathbf{U}_{j}^{(d)}$ in $\mathbb{R}_{*}^{2}$ and the truncated equation

$$
\widehat{f}_{j}^{(d)}(X)=0 .
$$

The support of the power asymptotic (2.4) consists of the two points $E_{2} \stackrel{\text { def }}{=}(0,1)$ and $(r, 0)$. Their convex hull is an edge, which is denoted by $\gamma_{1}^{(1)}$. The vector $(1, r)$ is normal to it. The normal cone $\mathbf{u}$ of a solution of the form (2.3) is the ray $\lambda \omega(1, r)$, where $\omega$ is defined by $(2.2)$ and $\lambda>0$.

Theorem 4 ([13, Ch. VI, Theorem 1.1]). If equation (2.1) has a solution of the form (2.3) such that $\mathbf{u}=\lambda \omega(1, r) \subset \mathbf{U}_{j}^{(d)}$, then the truncation (2.4) of the solution (2.3) is a solution of the truncated equation (2.8) corresponding to the face $\Gamma_{j}^{(d)}$.

This is a special case of Theorem 1 for $n=2$ and a power asymptotic.

Therefore, in order to find all the truncated solutions (2.4) of equation (2.1), one must calculate the support $\mathbf{S}(f)$, the polygon $\Gamma(f)$, all its faces $\Gamma_{j}^{(d)}$, and their normal cones $\mathbf{U}_{j}^{(d)}$. Then, for each truncated equation (2.8), one must find all its solutions (2.4) such that one of the vectors $\pm(1, r)$ belongs to the normal cone $\mathbf{U}_{j}^{(d)}$. If $d=0$, then this means that one of the vectors $\pm(1, r)=\omega R$ belongs to $\mathbf{U}_{j}^{(d)}$. If $d=1$, then this property is always satisfied. The value of $\omega$ is also determined in this process.

2.2. Solution of the truncated equation ([31, 32, and [33, §1]). Here we consider two cases separately: a vertex $\Gamma_{j}^{(0)}$, and an edge $\Gamma_{j}^{(1)}$.

Corresponding to the vertex $\Gamma_{j}^{(0)}=\{Q\}$ is the truncated equation (2.8) with the onepoint support $Q$ and with $d=0$. We set $g(X) \stackrel{\text { def }}{=} X^{-Q} \widehat{f}_{j}^{(0)}(X)$; then the solution (2.4) of equation (2.8) satisfies the equation $g(X)=0$. By substituting $y=c x^{r}$ into $g(X)$ we find that $g\left(x, c x^{r}\right)$ is independent of $x$ and $c$ and is a polynomial in $r$, that is, $g\left(x, c x^{r}\right) \equiv \chi(r)$, where $\chi(r)$ is the characteristic polynomial of the differential sum $\widehat{f}_{j}^{(0)}(X)$. Consequently, 
for the solution (2.4) of equation (2.8) the exponent $r$ is a root of the characteristic equation

$$
\chi(r) \stackrel{\text { def }}{=} g\left(x, x^{r}\right)=0,
$$

while the coefficient $c_{r}$ is arbitrary. From the roots $r_{i}$ of equation (2.9), we have to select only those for which one of the vectors $\omega(1, r)$, where $\omega= \pm 1$, belongs to the normal cone $\mathbf{U}_{j}^{(0)}$ of the vertex $\Gamma_{j}^{(0)}$. The corresponding expressions (2.4) with an arbitrary constant $c_{r}$ are candidates for the role of truncated solutions of equation (2.1). Here, according to (2.2), if $\omega=-1$, then $x \rightarrow 0$, and if $\omega=1$, then $x \rightarrow \infty$.

Corresponding to an edge $\Gamma_{j}^{(1)}$ we have the truncated equation (2.8) with $d=1$, whose normal cone $\mathbf{U}_{j}^{(1)}$ is the ray $\left\{\lambda N_{j}: \lambda>0\right\}$, where $N_{j}$ is an exterior normal vector to the edge $\Gamma_{j}^{(1)}$. The normal cone $\mathbf{u}$ of the truncated solution (2.4) intersects $\mathbf{U}_{j}^{(1)}$ only if the vector $\omega(1, r)$ belongs to $\mathbf{U}_{j}^{(1)}$. This uniquely determines the exponent $r$ of the truncated solution (2.4) and the value $\omega= \pm 1$ in (2.2). To determine the coefficient $c_{r}$ we must substitute the expression (2.4) into the truncated equation (2.8). After factoring out some power of $x$ we obtain the algebraic defining equation for the coefficient $c_{r}$,

$$
\widetilde{\widetilde{f}}\left(c_{r}\right) \stackrel{\text { def }}{=} x^{-s} \widehat{f}_{j}^{(1)}\left(x, c_{r} x^{r}\right)=0 .
$$

Each of its roots $c_{r}=c_{r}^{(i)} \neq 0$ has its own corresponding expression (2.4), which is a candidate for the role of a truncated solution of equation (2.1).

Thus, each truncated equation (2.8) has several suitable solutions (2.4) with $\mathbf{u} \subset \mathbf{U}_{j}^{(d)}$. We combine them into families that are continuous in $\omega, r, c_{r}$, and the parameters of equation (2.1); we denote these families by $\mathcal{F}_{j}^{(d)} k$, where $k=1,2, \ldots$

If we are not interested in all the solutions (2.3) of equation (2.1) but only in those whose normal cone $\mathbf{u}$ is contained in some given cone $\mathcal{K}$, then $\mathcal{K}$ is called the cone for the problem.

2.3. Critical numbers of a truncated solution ([31, 32, and [33, §1]). If a truncated solution (2.4) is found, then the change of variables

$$
y=c_{r} x^{r}+z
$$

reduces equation (2.1) to the form

$$
\widetilde{f}(x, z) \stackrel{\text { def }}{=} f\left(x, c x^{r}+z\right) \stackrel{\text { def }}{=} \mathcal{L}(x) z+h(x, z)=0,
$$

where $\widetilde{f}(x, z)$ is a differential sum and all the points $Q=\left(q_{1}, q_{2}\right)$ of its support $\mathbf{S}(\widetilde{f})$ have non-negative integer coordinate $q_{2}$. Here, $\mathcal{L}(x)$ is a linear differential operator and the support $\mathbf{S}(\mathcal{L} z)$ consists of a single point $(v, 1)$, which is a vertex $\widetilde{\Gamma}_{1}^{(0)}$ on the polygon $\Gamma(\widetilde{f})$; all points $Q=\left(q_{1}, q_{2}\right)$ of the support $\mathbf{S}(h)$ have coordinate $q_{2} \geq 0$ and $\mathbf{S}(h)$ does not contain the point $Q=(v, 1)$; the normal cone of the vertex $\widetilde{\Gamma}_{1}^{(0)}$ contains a vector $P=\left(p_{1}, p_{2}\right)$ with $p_{1} \omega>0$. For equation (2.12), the cone for the problem can be written in the form

$$
\mathcal{K}=\left\{s=p_{2} / p_{1}: s \omega<r \omega, p_{1} \omega>0\right\}
$$

that is, we seek only those solutions $z=c_{s} x^{s}+o\left(x^{s-r}\right)$ of equation (2.12) for which $s \in \mathcal{K}$. 
Recall that a differential sum $f(x, y)$ has the first variation (or the Fréchet derivative) $\delta f(x, y) / \delta y$, which has the following properties:

$$
\begin{gathered}
\frac{\delta\left(c x^{q_{1}} y^{q_{2}}\right)}{\delta y}=c q_{2} x^{q_{1}} y^{q_{2}-1}, \quad \frac{\delta\left(d^{l} y / d x^{l}\right)}{\delta y}=\frac{d^{l}}{d x^{l}}, \\
\frac{\delta(f+g)}{\delta y}=\frac{\delta f}{\delta y}+\frac{\delta g}{\delta y}, \quad \frac{\delta(f g)}{\delta y}=\left(\frac{\delta f}{\delta y}\right) g+f\left(\frac{\delta g}{\delta y}\right) .
\end{gathered}
$$

Theorem 5 (34). Suppose that (2.4) is a solution of the truncated equation (2.8) with $\mathbf{u} \in \mathbf{U}_{j}^{(d)}$. Then the operator $\mathcal{L}(x)$ in equation (2.12) satisfies

$$
\mathcal{L}(x)=\frac{\delta \widehat{f}_{j}^{(d)}(x, y)}{\delta y} \quad \text { at } \quad y=c_{r} x^{r}
$$

that is, it is equal to the first variation calculated at the solution (2.4). Furthermore, $\mathbf{S}(\mathcal{L} z)=(v, 1)$, where $v=\left\langle Q_{1},(1, r)\right\rangle-r$ with $Q_{1} \in \Gamma_{j}^{(d)}$.

Let $\nu(k)$ be the characteristic polynomial of the differential sum $\mathcal{L}(x) z$, that is,

$$
\nu(k)=x^{-v-k} \mathcal{L}(x) x^{k} .
$$

If $\nu(k) \not \equiv 0$, then the roots $k_{1}, \ldots, k_{s}$ of the polynomial $\nu(k)$ are called the eigenvalues of the truncated solution (2.4). Those of the real eigenvalues $k_{i}$ that belong to the cone for the problem (2.12) are called the critical numbers.

2.4. Statement of the problem concerning power-logarithmic expansions of solutions ([32, 35], and [33, $\S 1])$. If we seek solutions of equation $(2.12)$ with $\nu(k) \not \equiv 0$ in the form of a power series

$$
z=\sum c_{s} x^{s}, \quad \omega s<\omega r
$$

where $c_{s}=$ const $\in \mathbb{R}$, then such an expansion of solutions exists only under certain conditions. Here a basic condition is the absence of critical values. However, if these conditions are not imposed, then expansions of the form (2.16) are obtained with the $c_{s}$ being polynomials in $\ln x$.

Problem 2. Find the expansions

$$
z=\sum \beta_{s}(\ln x) x^{s}
$$

for solutions of equation (2.12), where the $\beta_{s}$ are polynomials in $\ln x$ and the exponents $s$ belong to (2.13), which is the cone for problem (2.12).

2.5. Support of an expansion of a solution ([32, 35], and [33, §3]). We consider equation (2.12) in the non-degenerate case where $\mathcal{L} \not \equiv 0$ and $\nu(k) \not \equiv 0$. Then to the term $\mathcal{L}(x) z$ there corresponds only one vertex $(v, 1)$ of the polygon $\Gamma(\widetilde{f})$. We translate the support $\mathbf{S}(\widetilde{f})$ by the vector $-(v, 1)$. Then the vertex $(v, 1)$ is translated to the origin. Let $\mathbf{S}^{\prime}{ }_{+}$be the set of finite sums of vectors $Q \in \mathbf{S}^{\prime} \stackrel{\text { def }}{=} \mathbf{S}(\widetilde{f})-(v, 1)$ and let

$$
\mathbf{K}=\mathbf{S}^{\prime}+\cap\left\{q_{2}=-1\right\} .
$$

Let $æ$ be the number of critical values $k_{i}$ of the truncated solution (2.4). We consider separately the cases with $æ=0 æ=1$.

Case $æ=0$.

Theorem 6. In the case $æ=0$ there is an expansion (2.16) of a solution of equation (2.12) in which all the exponents s belong to the set $\mathbf{K}$ and all the coefficients $c_{s}$ are uniquely determined. 
Lemma 1. Let $\Gamma_{j}^{(0)}$ be a vertex of the polygon $\Gamma(f)$ of equation (2.1) such that the truncated equation $\widehat{f}_{j}^{(0)}(X)=0$ has the solution (2.4), the vector $\omega(1, r)$ belongs to $\mathbf{U}_{j}^{(0)}$, and all the points of the translated support $\mathbf{S}(f)-\Gamma_{j}^{(0)}$ can be represented in the form $l R_{1}+m R_{2}$, where $l, m \geq 0$ are integers, while $R_{1}$ and $R_{2}$ are some vectors. Then the set (2.18) of equation (2.12) satisfies

$$
\mathbf{K} \subset\left\{s=r+l_{1} r_{1}+l_{2} r_{2}, \text { for integers } l_{1}, l_{2} \geq 0, l_{1}+l_{2}>0\right\},
$$

where $r_{1}=\left\langle(1, r), R_{1}\right\rangle$ and $r_{2}=\left\langle(1, r), R_{2}\right\rangle$.

A set $\mathbf{Z}$ of points in the plane $\mathbb{R}^{2}$ is called a lattice if for any two vectors $Q, Q^{\prime} \in \mathbf{Z}$ their sum $Q+Q^{\prime}$ and difference $Q-Q^{\prime}$ belong to $\mathbf{Z}$. Vectors $B_{1}$ and $B_{2}$ form a basis of the lattice $\mathbf{Z}$ if any point $Q$ of $\mathbf{Z}$ can be represented in the form $Q=n_{1} B_{1}+n_{2} B_{2}$, where $n_{1}, n_{2} \in \mathbb{Z}$, that is, $n_{1}, n_{2}$ are integers. Let $\mathbf{S}$ be some set in $\mathbb{R}^{2}$ and let $Q \in \mathbf{S}$. The set of points $\mathbf{S}^{\prime} \stackrel{\text { def }}{=} \mathbf{S}-Q$ is called a shifted set $\mathbf{S}$. For the truncated solution (2.4), the support $\mathbf{s}$ consists of the two points $Q_{1}=(0,1)$ and $Q_{2}=(r, 0)$. Therefore the shifted support $\mathbf{s}^{\prime}$ consists of the points 0 and $Q_{2}-Q_{1}=(r,-1)$.

Remark 2. If the shifted supports $\mathbf{S}^{\prime}(f)$ of equation (2.1), and $\mathbf{s}^{\prime}$ of the truncated solution (2.4), are contained in some lattice $\mathbf{Z}$, then

$$
\mathbf{K} \subset \mathbf{Z} \cap\left\{q_{2}=-1\right\} \cap \mathcal{K} .
$$

Case $æ=1$. Suppose that $k_{1}$ is the unique critical value. We denote by $\mathbf{S}_{+}{ }_{+}\left(k_{1}\right)$ the set of finite sums of vectors $Q \in \mathbf{S}^{\prime} \stackrel{\text { def }}{=} \mathbf{S}(\widetilde{f})-(v, 1)$ and the vector $\left(k_{1},-1\right)$ and set

$$
\mathbf{K}\left(k_{1}\right)=\mathbf{S}^{\prime}{ }_{+}\left(k_{1}\right) \cap\left\{q_{2}=-1\right\} .
$$

Theorem 7. If in the case $æ=1$ the unique critical value $k_{1}$ does not belong to the set $\mathbf{K}$, then there is an expansion (2.16) of a solution of equation (2.12), where the exponents $s$ belong to the set $\mathbf{K}\left(k_{1}\right)$, the coefficient $c_{k_{1}}$ is arbitrary, and the other coefficients $c_{s}$ are uniquely determined.

Lemma 2. If the set (2.18) has the form

$$
\mathbf{K}=\{k=r+l \widetilde{r}, \text { for integers } l>0\},
$$

then the set (2.20) has the form

$$
\mathbf{K}\left(k_{1}\right)=\left\{k=r+l \widetilde{r}+m\left(k_{1}-r\right), \text { for integers } l, m \geq 0, l+m>0\right\} .
$$

Remark 3. If the set $\mathbf{S}^{\prime}=\mathbf{S}(\tilde{f})-(v, 1)$ and the point $\left(k_{1},-1\right)$ are contained in some lattice $\mathbf{Z}\left(k_{1}\right)$, then

$$
\mathbf{K}\left(k_{1}\right) \subset \mathbf{Z}\left(k_{1}\right) \cap\left\{q_{2}=-1\right\} \cap \mathcal{K} .
$$

Theorem 8. If the set $\mathbf{S}(\widetilde{f})$ has no accumulation points on the plane $\mathbb{R}^{2}$, then the sets (2.18) and (2.20) have no accumulation points on the real line $\mathbb{R}$.

Theorem 9. If in the case $æ=1$ the unique critical value $k_{1}$ belongs to $\mathbf{K}$, then equation (2.12) has a formal solution of the form (2.17),

$$
z=z^{*}(x) \stackrel{\text { def }}{=} \sum \beta_{s} x^{s}, \quad s \in \mathbf{K},
$$

where the $\beta_{s}$ are constants if $\omega s<\omega k_{1}$, the $\beta_{s}$ are polynomials in $\ln x$ if $\omega s \geq \omega k_{1}$, and $\beta_{k_{1}}$ is a linear polynomial in $\ln x$ with an arbitrary constant term.

The maximum of the orders $l$ of all the derivatives $d^{l} y / d x^{l}$ involved in a differential monomial $a(X)$ is called its differentiation order and is denoted by $\pi(a)$. The differentiation order $\pi(f)$ of the sum (1.1) is defined as the greatest of the differentiation orders $\pi\left(a_{i}\right)$ of its monomials $a_{i}(X)$. 
Theorem 10 (34). Suppose that under the hypotheses of Theorems 6 and 7, in the sum (2.12) $\pi(\mathcal{L} z)=\pi(\widetilde{f})$; then the expansion (2.16) is absolutely convergent for sufficiently small $|x| \neq 0$ as $x \rightarrow 0$, and for sufficiently small $|x|^{-1} \neq 0$ as $x \rightarrow \infty$ (that is, for sufficiently small $|x|^{-\omega} \neq 0$ ).

2.6. Statement of the problem concerning non-power asymptotics of solutions ([32, 36], and [33, §5]). Suppose that $x \rightarrow 0$ or $x \rightarrow \infty$. Two functions $\varphi(x)$ and $\psi(x)$ are said to be weakly (asymptotically) equivalent if

$$
\frac{\psi(x)}{\varphi(x)} \rightarrow 1
$$

Then the function $\varphi(x)$ is a weak asymptotic of the function $\psi(x)$, and vice versa. We denote the $k$-multiple exponential and logarithm by $\exp ^{(k)}\{x\}$ and $\ln ^{(k)} x$, respectively, that is,

$$
\exp ^{(k)}\{x\} \stackrel{\text { def }}{=} \exp \{\exp \{\ldots(\exp \{x\}) \ldots\}\} \quad \text { and } \quad \ln ^{(k)} x \stackrel{\text { def }}{=} \ln (\ln (\ldots(\ln x) \ldots)) .
$$

Problem 3. For solutions of equation (2.1), where $f(x, y)$ is a differential sum, find all (weak) asymptotics of the form

$$
y=c x^{r}(\exp \{x\})^{s_{1}} \ldots\left(\exp ^{(k)}\{x\}\right)^{s_{k}}(\ln x)^{t_{1}} \ldots\left(\ln ^{(l)} x\right)^{t_{l}},
$$

where $c=$ const $\in \mathbb{R}, c \neq 0$, and $r, s_{i}, t_{i}=$ const $\in \mathbb{R}$.

In $\S 2.2$ we expounded a method for calculating all the power asymptotics, that is, asymptotics of the form (2.4). Therefore here we focus on calculating all the non-power asymptotics of solutions, that is, not having the form (2.4).

Let $y=\varphi(x)$ be a solution of equation (2.1), and suppose that the function $\varphi(x)$ has order $r$. Then the normal cone $\mathbf{u}$ of this solution is the ray

$$
\mathbf{u}=\left\{\begin{aligned}
\lambda(0,-1) & \text { if } \quad r=-\infty \\
\lambda \omega(1, r) & \text { if } \quad r \in \mathbb{R} ; \\
\lambda(0,1) & \text { if } \quad r=+\infty, \quad \lambda>0 .
\end{aligned}\right.
$$

The notion of the cone for the problem also applies to solutions when the normal cone $\mathbf{u}$ has this definition.

Two functions $\varphi(x)$ and $\psi(x)$ are strongly (asymptotically) equivalent if

$$
\varphi(x)=\psi(x)\left[1+o\left(|x|^{\omega \varepsilon}|\psi(x)|^{\omega_{2} \varepsilon}\right)\right]
$$

for some $\varepsilon<0$, where

$$
\omega_{2}=\left\{\begin{array}{rll}
-1 & \text { if } \quad \psi(x) \rightarrow 0, \\
0 & \text { if } \quad \psi(x) \rightarrow \text { const } \neq 0, \\
1 & \text { if } \quad \psi(x) \rightarrow \infty .
\end{array}\right.
$$

Then the function $\varphi(x)$ is a strong asymptotic of the function $\psi(x)$, and vice versa. This is a special case of the strong asymptotic equivalence introduced in $\S 1$ of this chapter.

Theorem 11 ([13, Ch. VI, Theorem 1.1]). If $\mathbf{u}$ is the normal cone of a solution $y=\varphi(x)$ of equation (2.1), then the truncated equation (2.8) such that $\mathbf{u} \subset \mathbf{U}_{j}^{(d)}$ has a strongly asymptotically equivalent solution $y=\psi(x)$.

This is Theorem 1 for $n=2$.

Thus, Problem 3 reduces to finding all the truncated equations and their normal cones and to a finite number of the following problems. 
Problem 4. For the truncated equation (2.8), find all the (weak) asymptotics of its solutions $y=\psi(x)$ with $\omega(1, r) \in \mathbf{U}_{j}^{(d)}$.

The polygon $\Gamma(f)$ corresponds to equation (2.1), and $\Gamma_{j}^{(d)}$, one of its edges or vertices corresponds to the truncated equation (2.8). Below we consider separately three methods for the reduction of Problem 4, depending on the inclination of the edge.

2.7. A vertical edge $\Gamma_{j}^{(1)}\left(\left[32\right.\right.$, 36], and [33, §5]). If the edge $\Gamma_{j}^{(1)}$ is vertical, then its normal cone is

$$
\mathbf{U}_{j}^{(1)}=\lambda \omega(1,0), \quad \lambda>0,
$$

and the coordinate $q_{1}$ is the same for all points $Q=\left(q_{1}, q_{2}\right) \in \Gamma_{j}^{(1)}$. We set

$$
g(x, y)=x^{-q_{1}} \widehat{f}_{j}^{(d)}(x, y) ;
$$

then the support $\mathbf{S}(g)$ lies on the coordinate axis $q_{1}=0$. According to (2.24) all the power solutions (2.4) with $\omega(1, r) \in \mathbf{U}_{j}^{(1)}$ are constants $y=y^{0}=$ const, where $y^{0}$ is a root of the defining equation

$$
\widetilde{g}(y) \stackrel{\text { def }}{=} g(0, y)=0 .
$$

A root $y^{0}$ of equation (2.26) is called repeated if the derivative $d \widetilde{g}(y) / d y$ vanishes at it.

To find the non-power solutions of equation (2.8) we make the logarithmic transformation

$$
\xi \stackrel{\text { def }}{=} \ln x .
$$

Then, according to Theorem 2.4 in [13, Ch. VI], the differential sum $g(x, y)$ is transformed into a differential sum $h(\xi, y) \stackrel{\text { def }}{=} g(x, y)$, and equation $(2.8)$ takes the form

$$
h(\xi, y)=0 .
$$

It is clear from (2.27) that $\xi \rightarrow \infty$ as $x \rightarrow 0$ and as $x \rightarrow \infty$; that is, for equation (2.28) we obtain Problems 1 and 2, and the cone for the problem is

$$
p_{1} \geq 0 \text {. }
$$

Theorem 12. Finite limiting values $y^{0} \neq 0$ of non-constant solutions of equation (2.28) are repeated solutions of equation (2.26).

Suppose that the edge $\Gamma_{j}^{(1)}$ connects the vertices $\Gamma_{j-1}^{(0)}=\left(q_{1}^{\prime}, q_{2}^{\prime}\right)$ and $\Gamma_{j}^{(0)}=\left(q_{1}^{\prime}, q_{2}^{\prime \prime}\right)$, where $q_{2}^{\prime}<q_{2}^{\prime \prime}$ are integers. We say that equation (2.26) has an infinite root if the degree of the polynomial in (2.26) is less than $q_{2}^{\prime \prime}$, and a zero root if the lowest degree in $y$ of the polynomial in $(2.26)$ is greater than $q_{2}^{\prime}$.

Theorem 13. If equation (2.26) does not have an infinite (zero) root, then equation (2.28) has no solutions tending to infinity (zero).

Note that the truncation of equation $(2.28)$ with respect to the vector $(1,0)$ is equation (2.26), that is, $\widetilde{g}(y) \equiv \widehat{h}(\xi, y)$. To find the solutions of equation (2.28) with infinite or zero limiting values of $y$, for equation (2.28) one must select the truncated equations corresponding to the cone for the problem (2.29). To find the solutions of equation (2.28) with finite limiting values one must find all the repeated roots of equation (2.26). Let $y^{0}$ be such a repeated root. Use the translation $y=y^{0}+z$ to place this root at the origin. Equation (2.28) takes the form

$$
\widetilde{h}(\xi, z) \stackrel{\text { def }}{=} h\left(\xi, y^{0}+z\right)=0 .
$$


Now for equation (2.30) select its truncated equations with the cone for the problem $p_{1} \geq 0, p_{2} \leq 0$. This is again Problem 1 .

2.8. An inclined edge $\Gamma_{j}^{(1)}([32,36]$, and [33, $\left.\S 5]\right)$. Suppose that the vector $(1, r)$ with $r \neq 0$ is normal to the edge $\Gamma_{j}^{(1)}$.

Theorem 14 ([13, Ch. VI, Theorem 2.2]). The power transformation

$$
y=z x^{r}
$$

reduces equation (2.8) to the form

$$
\widetilde{f}(x, z) \stackrel{\text { def }}{=} \widehat{f}_{j}^{(1)}\left(x, z x^{r}\right)=0,
$$

where $\tilde{f}(x, z)$ is a differential sum and its support $\mathbf{S}(\widetilde{f})$ is situated on the vertical line $q_{1}=$ const.

Thus, the power transformation (2.31) reduces the case of an inclined edge to the case of a vertical edge. If we substitute $y=c x^{r}$ into the truncated equation (2.8), then for the coefficient $c$ we obtain the defining equation

$$
x^{s} \widetilde{\widetilde{f}}(c) \stackrel{\text { def }}{=} \widehat{f}_{j}^{(1)}\left(x, c x^{r}\right)=0,
$$

where $\widetilde{\widetilde{f}}(c)$ is a polynomial in the coefficient $c$. Here this polynomial plays the same role as the polynomial $\widetilde{g}(y)$ in equation $(2.26)$.

Remark 4. In particular, if this polynomial has no infinite, zero, or repeated roots, then equation (2.8) has no suitable non-power solutions, that is, there is no need in the transformation (2.31) and further investigation.

2.9. A horizontal edge $\boldsymbol{\Gamma}_{\boldsymbol{j}}^{(\mathbf{1})}([32,36$, and [33, $\S 5])$. In this case all the points $Q=$ $\left(q_{1}, q_{2}\right)$ of the edge $\Gamma_{j}^{(1)}$ have the same coordinate $q_{2}$. We set

$$
g(x, y) \stackrel{\text { def }}{=} y^{-q_{2}} \widehat{f}_{j}^{(1)}(x, y)
$$

and perform the logarithmic transformation

$$
\eta=\frac{d \ln y}{d x} .
$$

According to Theorem 2.4 in [13, Ch. VI], under this transformation the differential sum $g(x, y)$ becomes a differential sum $h(x, \eta) \stackrel{\text { def }}{=} g(x, y)$, and equation $(2.8)$ takes the form

$$
h(x, \eta)=0 .
$$

For equation (2.35) we now obtain Problem 1 where the cone for the problem is

$$
p_{1}+p_{2} \geq 0
$$




\section{CHAPTER II. FLOW OF A VISCOUS INCOMPRESSIBLE FLUID PAST A NEEDLE}

\section{Transforming the system of Navier-Stokes Equations}

We consider the system of Navier-Stokes equations [37, Ch. VIII, §76], 38, Ch.3, $\S 3.3]$, which describes a steady flow of a viscous incompressible fluid in space,

$$
\begin{aligned}
u \frac{\partial u}{\partial x}+v \frac{\partial u}{\partial y}+w \frac{\partial u}{\partial z}+\frac{1}{\rho} \frac{\partial p}{\partial x} & =\nu\left(\frac{\partial^{2} u}{\partial x^{2}}+\frac{\partial^{2} u}{\partial y^{2}}+\frac{\partial^{2} u}{\partial z^{2}}\right), \\
u \frac{\partial v}{\partial x}+v \frac{\partial v}{\partial y}+w \frac{\partial v}{\partial z}+\frac{1}{\rho} \frac{\partial p}{\partial y} & =\nu\left(\frac{\partial^{2} v}{\partial x^{2}}+\frac{\partial^{2} v}{\partial y^{2}}+\frac{\partial^{2} v}{\partial z^{2}}\right), \\
u \frac{\partial w}{\partial x}+v \frac{\partial w}{\partial y}+w \frac{\partial w}{\partial z}+\frac{1}{\rho} \frac{\partial p}{\partial z} & =\nu\left(\frac{\partial^{2} w}{\partial x^{2}}+\frac{\partial^{2} w}{\partial y^{2}}+\frac{\partial^{2} w}{\partial z^{2}}\right), \\
\frac{\partial u}{\partial x}+\frac{\partial v}{\partial y}+\frac{\partial w}{\partial z} & =0 .
\end{aligned}
$$

Here $x, y$, and $z$ are Cartesian coordinates; $u, v$, and $w$ are the components of the flow velocity vector along the $x-, y$-, and $z$-axis, respectively; $p$ is the pressure; $\rho$ is the density; $\nu$ is the kinematic viscosity coefficient; $\rho, \nu=$ const $\neq 0$.

After passing to cylindrical coordinates the system takes the following form (37, Ch. VIII, §76], [38, Appendix 2]):

$$
\begin{aligned}
V_{r} \frac{\partial V_{r}}{\partial r}+\frac{V_{\varepsilon}}{r} \frac{\partial V_{r}}{\partial \varepsilon}+V_{x} \frac{\partial V_{r}}{\partial x}-\frac{V_{\varepsilon}^{2}}{r}+\frac{1}{\rho} \frac{\partial p}{\partial r} & =\nu\left(\nabla^{2} V_{r}-\frac{V_{r}}{r^{2}}-\frac{2}{r^{2}} \frac{\partial V_{\varepsilon}}{\partial \varepsilon}\right), \\
V_{r} \frac{\partial V_{\varepsilon}}{\partial r}+\frac{V_{\varepsilon}}{r} \frac{\partial V_{\varepsilon}}{\partial \varepsilon}+V_{x} \frac{\partial V_{\varepsilon}}{\partial x}+\frac{V_{r} V_{\varepsilon}}{r}+\frac{1}{\rho} \frac{\partial p}{\partial \varepsilon} \frac{1}{r} & =\nu\left(\nabla^{2} V_{\varepsilon}+\frac{2}{r^{2}} \frac{\partial V_{r}}{\partial \varepsilon}-\frac{V_{\varepsilon}}{r^{2}}\right), \\
V_{r} \frac{\partial V_{x}}{\partial r}+\frac{V_{\varepsilon}}{r} \frac{\partial V_{x}}{\partial \varepsilon}+V_{x} \frac{\partial V_{x}}{\partial x}+\frac{1}{\rho} \frac{\partial p}{\partial x} & =\nu\left(\nabla^{2} V_{x}\right), \\
\frac{\partial\left(r V_{r}\right)}{\partial r}+\frac{\partial V_{\varepsilon}}{\partial \varepsilon}+\frac{\partial\left(r V_{x}\right)}{\partial x} & =0
\end{aligned}
$$

where

$$
\nabla^{2} \stackrel{\text { def }}{=} \frac{\partial^{2}}{\partial r^{2}}+\frac{1}{r} \frac{\partial}{\partial r}+\frac{1}{r^{2}} \frac{\partial^{2}}{\partial \varepsilon^{2}}+\frac{\partial^{2}}{\partial x^{2}} ;
$$

$x, r$, and $\varepsilon$ are the cylindrical coordinates $y=r \sin \varepsilon, z=r \cos \varepsilon ; V_{x}, V_{r}$, and $V_{\varepsilon}$ are the components of the flow velocity vector. For an axisymmetric flow one can eliminate the angular component $\varepsilon$ and the corresponding component $V_{\varepsilon}$ of the flow velocity vector from the system. Then the system (1.2) takes the form

$$
\begin{aligned}
V_{r} \frac{\partial V_{r}}{\partial r}+V_{x} \frac{\partial V_{r}}{\partial x}+\frac{1}{\rho} \frac{\partial p}{\partial r} & =\nu\left(\frac{\partial^{2} V_{r}}{\partial r^{2}}+\frac{1}{r} \frac{\partial V_{r}}{\partial r}+\frac{\partial^{2} V_{r}}{\partial x^{2}}-\frac{V_{r}}{r^{2}}\right), \\
V_{r} \frac{\partial V_{x}}{\partial r}+V_{x} \frac{\partial V_{x}}{\partial x}+\frac{1}{\rho} \frac{\partial p}{\partial x} & =\nu\left(\frac{\partial^{2} V_{x}}{\partial r^{2}}+\frac{1}{r} \frac{\partial V_{x}}{\partial r}+\frac{\partial^{2} V_{x}}{\partial x^{2}}\right) \\
\frac{\partial\left(r V_{r}\right)}{\partial r}+\frac{\partial\left(r V_{x}\right)}{\partial x} & =0 .
\end{aligned}
$$

Using the third equation we introduce the stream function $\psi$ by the formulae

$$
V_{x}=\frac{1}{r} \frac{\partial \psi}{\partial r}, \quad V_{r}=-\frac{1}{r} \frac{\partial \psi}{\partial x} .
$$


Then the system (1.3) takes the form

$$
\begin{aligned}
f_{1} \stackrel{\text { def }}{=} & {\left[\frac{1}{r} \frac{\partial \psi}{\partial x} \frac{\partial}{\partial r}\left(\frac{1}{r} \frac{\partial \psi}{\partial x}\right)-\frac{1}{r} \frac{\partial \psi}{\partial r} \frac{\partial}{\partial x}\left(\frac{1}{r} \frac{\partial \psi}{\partial x}\right)\right]+\frac{1}{\rho} \frac{\partial p}{\partial r} } \\
& +\nu\left[\frac{\partial}{\partial r}\left(\frac{1}{r} \frac{\partial^{2} \psi}{\partial x \partial r}\right)+\frac{\partial^{2}}{\partial x^{2}}\left(\frac{1}{r} \frac{\partial \psi}{\partial x}\right)\right]=0, \\
f_{2} \stackrel{\text { def }}{=} & {\left[-\frac{1}{r} \frac{\partial \psi}{\partial x} \frac{\partial}{\partial r}\left(\frac{1}{r} \frac{\partial \psi}{\partial r}\right)+\frac{1}{r} \frac{\partial \psi}{\partial r} \frac{\partial}{\partial x}\left(\frac{1}{r} \frac{\partial \psi}{\partial r}\right)\right]+\frac{1}{\rho} \frac{\partial p}{\partial x} } \\
& -\nu\left[\frac{1}{r} \frac{\partial}{\partial r}\left(r \frac{\partial}{\partial r}\left(\frac{1}{r} \frac{\partial \psi}{\partial r}\right)\right)+\frac{\partial^{2}}{\partial x^{2}}\left(\frac{1}{r} \frac{\partial \psi}{\partial r}\right)\right]=0 .
\end{aligned}
$$

In the notation of Chapter I, $\S 1$,

$$
x_{1}=x, \quad x_{2}=r, \quad x_{3}=\psi, \quad x_{4}=p .
$$

\section{First approximations to the SOlution at infinity}

We now consider a steady flow of a viscous incompressible fluid past a semi-infinite needle occupying the half-line $\{x, y, z: x \geq 0, y=z=0\}$ in the direction of the positive $x$-axis. Such a flow is described by the system of Navier-Stokes equations (1.1) with the boundary conditions

$$
\begin{aligned}
u=u_{\infty}, \quad v=w=0, \quad p=p_{0} & \text { at } x=-\infty, \quad \text { where } u_{\infty}, p_{0}=\text { const } \neq 0 \\
u=v=w=0 & \text { for } x \geq 0, y=z=0 .
\end{aligned}
$$

In cylindrical coordinates this flow is described by the system (1.3) with the boundary conditions

$$
\begin{gathered}
V_{x}=u_{\infty}, \quad V_{r}=0, \quad p=p_{0} \quad \text { at } x=-\infty ; \\
V_{x}=V_{r}=0 \quad \text { for } x \geq 0, r=0 .
\end{gathered}
$$

According to (1.4), an axisymmetric flow past the needle is also described by the system (1.5) with the following boundary conditions at infinity and at the needle:

$$
\begin{gathered}
\psi=u_{\infty} \frac{r^{2}}{2}, \quad p=p_{0} \quad \text { at } x=-\infty, \quad \text { where } u_{\infty}, p_{0}=\text { const } \neq 0 ; \\
\frac{\partial \psi}{\partial x}=\frac{\partial \psi}{\partial r}=\frac{\partial^{2} \psi}{\partial x \partial r}=\frac{\partial^{2} \psi}{\partial r^{2}}=0 \quad \text { for } x \geq 0, r=0 .
\end{gathered}
$$

The change of coordinates

$$
x=2 \nu u_{\infty}^{-1} \widetilde{x}, \quad r=2 \nu u_{\infty}^{-1} \widetilde{r}, \quad \psi=2 \nu^{2} u_{\infty}^{-1} \widetilde{\psi}, \quad p=\frac{\widetilde{p} u_{\infty}^{2}}{4}
$$

reduces this problem to the problem considered below with $u_{\infty}=2, \quad \nu=1$ and with new $p_{0}=$ const $\neq 0$.

Lemma 1. The boundary conditions

$$
\psi=r^{2}, \quad p=p_{0} \quad \text { as } r \rightarrow+\infty, \quad \text { where } p_{0}=\text { const },
$$

hold for $x \in(-\infty,+\infty)$.

Proof. We observe that the functions $\psi=r^{2}, p=p_{0}$ annihilate each of the terms in equations (1.5). Consequently, the functions (2.5) are solutions of any truncated system for (1.5). As in [13, Ch. VI, §6.2], by moving over the truncated systems that exist for $r \rightarrow \infty$ and $-\infty<x<0$, we can extend the boundary condition (2.3) to the case $x>0, r \rightarrow \infty$, that is, we can obtain the condition (2.5). To do this, we consider all the truncated systems of the system (1.5) that correspond to the boundary conditions (2.5). 
TABLE 1. Selection of the truncated system. Here, $\Delta_{k} \stackrel{\text { def }}{=}\left\langle\widetilde{P}, \widetilde{Q}_{k}\right\rangle$.

\begin{tabular}{|c|c|c|c|c|c|}
\hline$i$ & $k$ & $Q_{k}$ & $\widetilde{Q}_{k}$ & $\Delta_{k}$ & $\mathrm{~T}$ \\
\hline \multirow[t]{4}{*}{ 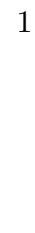 } & 1 & $-2,-3,2,0$ & $-2, \quad 1$ & -3 & \\
\hline & 2 & $0,-1,0,1$ & $0,-1$ & -1 & + \\
\hline & 3 & $-1,-3,1,0$ & $-1,-1$ & -3 & \\
\hline & 4 & $-3,-1,1,0$ & $-3, \quad 1$ & -5 & \\
\hline \multirow[t]{4}{*}{2} & 5 & $-1,-4,2,0$ & $-1, \quad 0$ & -2 & + \\
\hline & 6 & $-1, \quad 0,0,1$ & $-1, \quad 0$ & -2 & + \\
\hline & 7 & $0,-4,1,0$ & $0,-2$ & -2 & + \\
\hline & 8 & $-2,-2,1,0$ & $-2, \quad 0$ & -4 & \\
\hline
\end{tabular}

The supports of the equations of the system (1.5) are presented in Table 1 Its first column contains the number $i$ of the equation $f_{i}=0$, the second column contains the number $k$ of the point $Q_{k}$ of the support, the third column contains the actual points $\widetilde{Q}_{k}$ in the supports $\mathbf{S}\left(f_{i}\right)$. In the equations of the system (1.5), terms with the same vectorial exponent are combined in the square brackets.

It is clear from the boundary condition (2.5) that as $r \rightarrow+\infty$ there are boundary conditions of the same form as (1.16) in Chapter I:

$$
f_{3} \stackrel{\text { def }}{=} \psi-r^{2}=0, \quad f_{4} \stackrel{\text { def }}{=} p-p_{0}=0 .
$$

To each of them corresponds a support consisting of two points. Namely, $\mathbf{S}\left(f_{3}\right)$ consists of the points $Q_{9}=(0,0,1,0)$ and $Q_{10}=(0,2,0,0)$; and $\mathbf{S}\left(f_{4}\right)$ consists of the points $Q_{11}=$ $(0,0,0,1)$ and $Q_{12}=(0,0,0,0)$. According to Chapter I, Theorem 2 the vector $P=$ $\left(p_{1}, p_{2}, p_{3}, p_{4}\right)$ must satisfy the conditions $\left\langle Q_{9}, P\right\rangle=\left\langle Q_{10}, P\right\rangle$ and $\left\langle Q_{11}, P\right\rangle=\left\langle Q_{12}, P\right\rangle$, that is,

$$
p_{3}=2 p_{2}, \quad p_{4}=0 .
$$

In the notation of Chapter I, here, $m^{\prime}=l=2$ and $R_{3}^{\prime}=(0,2), R_{4}^{\prime}=(0,0)$. According to the conditions (2.6) on the vector $P$ obtained above, applying Chapter I, Theorem 2 the vectors $Q_{k}$ can be projected onto the plane $\widetilde{Q}=\left(\widetilde{q}_{1}, \widetilde{q}_{2}\right)$ by the formulae $\widetilde{q}_{1}=q_{1}$, $\widetilde{q}_{2}=q_{2}+2 q_{3}$, while the value of $q_{4}$ is neglected. The fourth column of Table 1 contains the projections $\widetilde{Q}_{k}=\left(q_{1}, q_{2}+2 q_{3}\right)$ of the vectors $Q_{k}$. The projections $\widetilde{\mathbf{S}}\left(f_{1}\right)$ and $\widetilde{\mathbf{S}}\left(f_{2}\right)$ of the supports of the equations in (1.5), their convex hulls $\widetilde{\Gamma}_{1}, \widetilde{\Gamma}_{2}$, and their normal cones are represented in Figure 2.

Corresponding to each vector $\widetilde{P}=\left(p_{1}, p_{2}\right)$ there is a truncated system

$$
\widehat{f}_{i \widetilde{P}}(X)=0, \quad i=1,2,
$$

of the system (1.5), where $\widehat{f}_{i \widetilde{P}}(X)$ contains those terms in $\mathbf{S}\left(f_{i}\right)$ for which the scalar product $\langle\widetilde{P}, \widetilde{Q}\rangle$ has the greatest value for this $i$. The vector $\widetilde{P}=(1,0)$ and the corresponding truncated system (2.7) correspond to the boundary condition (2.3) with $x \rightarrow-\infty$. According to Figures 2 and 3. the same truncated system corresponds to the vectors $\widetilde{P}=\left(p_{1}, p_{2}\right)$ with $p_{1}>p_{2} / 2>0$, that is, to the values $x<0$ with $\ln |x|>(1 / 2) \ln r>0$ as $r \rightarrow \infty$. 

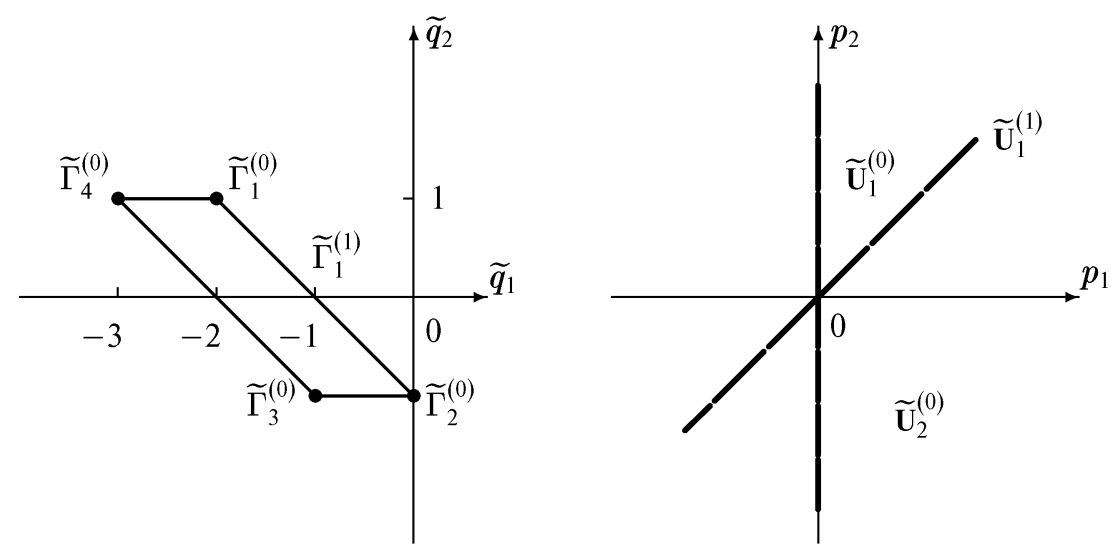

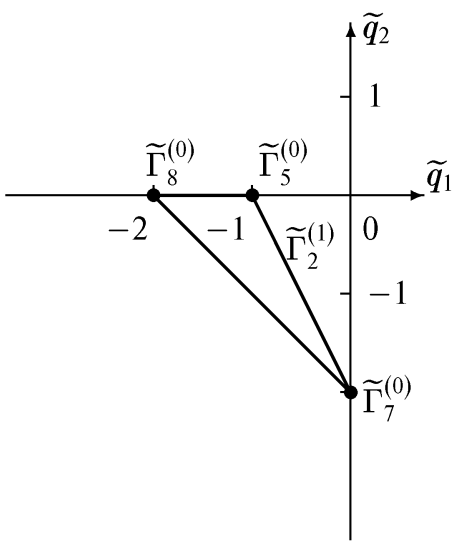

a)

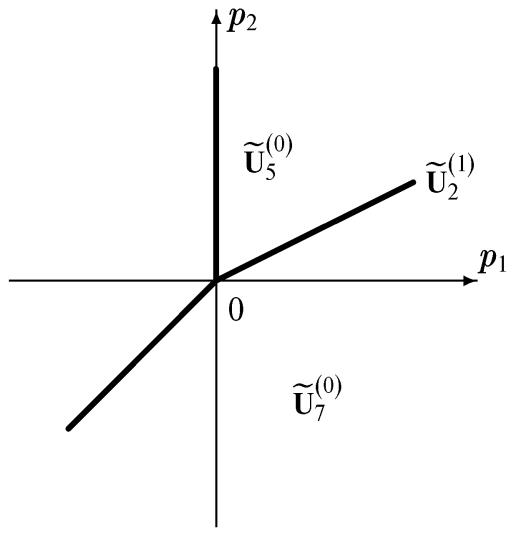

b)

FiguRE 2. (a) The projections of the supports of the equations of the system (1.5), and (b) the normal cones of the projections.

Consequently, the boundary conditions (2.5) hold for these $x$ and $r$. As $r \rightarrow \infty$, the rotation of the vector $\widetilde{P}$ in the positive direction from $(1,0)$ to $(0,1)$ corresponds to the motion along $x$ from $-\infty$ to 0 .

Moving over the corresponding truncated systems (2.7), we extend the condition (2.5) to the values $x \in(-\infty, 0)$.

Moving over the same truncated systems in the opposite direction, that is, from the vector $\widetilde{P}=(0,1)$ to the vector $\widetilde{P}=(2,1)$, we extend condition $(2.5)$ to $x \in[0,+\infty)$.

Traditionally, boundary conditions at the outer boundary of the boundary layer are taken in the flow that is not perturbed by the obstacle the flow is passing (in our case, the needle). Consequently, the boundary conditions (2.3) are extended to the boundary conditions (2.5). But this procedure is based on physical considerations. In Lemma 1 this was done purely mathematically.

Lemma 2. For the system (1.5), the truncated system corresponding to the boundary layer on the needle and the boundary conditions (2.5) is unique. It has the normal vector 
$P=(2,1,2,0)$ and has the form

$$
\begin{gathered}
\widehat{f}_{11} \stackrel{\text { def }}{=} \frac{1}{\rho} \frac{\partial p}{\partial r}=0, \\
\widehat{f_{21} \stackrel{\text { def }}{=}}\left[-\frac{1}{r} \frac{\partial \psi}{\partial x} \frac{\partial}{\partial r}\left(\frac{1}{r} \frac{\partial \psi}{\partial r}\right)+\frac{1}{r} \frac{\partial \psi}{\partial r} \frac{\partial}{\partial x}\left(\frac{1}{r} \frac{\partial \psi}{\partial r}\right)\right] \\
+\frac{1}{\rho} \frac{\partial p}{\partial x}-\frac{1}{r} \frac{\partial}{\partial r}\left(r \frac{\partial}{\partial r}\left(\frac{1}{r} \frac{\partial \psi}{\partial r}\right)\right)=0,
\end{gathered}
$$

and the self-similar coordinates $\xi, h(\xi)$, and $p(\xi)$ for the problem (2.4), (2.5), (2.8), (2.9) are

$$
\xi=\frac{r^{2}}{x}, \quad h(\xi)=\frac{\psi}{x}, \quad p(\xi)=p .
$$

Proof. The needle is described as $x \geq 0, r=0$. Near the needle, as $x \rightarrow+\infty$ and $r \rightarrow 0$, we have $p_{1}>0$ and $p_{2}<0$. Consequently, the IVth quadrant of the plane $\left(p_{1}, p_{2}\right)$ corresponds to the needle. As $x \rightarrow+\infty$ and $r \rightarrow+\infty$, the boundary conditions (2.5) mean that $p_{1}, p_{2}>0$; that is, there are points in the Ist quadrant of the $\left(p_{1}, p_{2}\right)$-plane corresponding to them. We are interested in the faces of the projections $\widetilde{\Gamma}_{1}$ and $\widetilde{\Gamma}_{2}$ whose extended normal cone contains both the IVth quadrant and points in the Ist quadrant of the plane $\left(p_{1}, p_{2}\right)$. The superimposition of the pictures of the normal cones of the projections is shown in Figure 3, It is clear that the IVth quadrant and points in the Ist quadrant are contained only in the extended normal cone of the system $\check{\mathbf{U}}_{J}^{D}=\check{\mathbf{U}}_{2}^{(1)} \cap \check{\mathbf{U}}_{2}^{(0)}$. The vector $\widetilde{P}=(2,1)$ is a direction vector of the normal cone $\widetilde{\mathbf{U}}_{2}^{(1)}$. From the vector $\widetilde{P}=\left(p_{1}, p_{2}\right)$ we reconstruct the vector $P=\left(p_{1}, p_{2}, p_{3}, p_{4}\right)$ according to the equalities $(2.6)$ and obtain in the original coordinates $\left(p_{1}, p_{2}, p_{3}, p_{4}\right)$ the vector $P=(2,1,2,0)$. The faces of the supports $\mathbf{S}\left(f_{1}\right)$ and $\mathbf{S}\left(f_{2}\right)$ correspond to this vector $P$ and contain the points $Q_{1}, Q_{2}, Q_{3}, Q_{6}$. The truncated system (2.8), (2.9) corresponds to these points.

The fifth column of Table 1 contains the values of the scalar products

$$
\Delta_{k}=\left\langle\widetilde{P}, \widetilde{Q}_{k}\right\rangle=\left\langle P, Q_{k}\right\rangle
$$

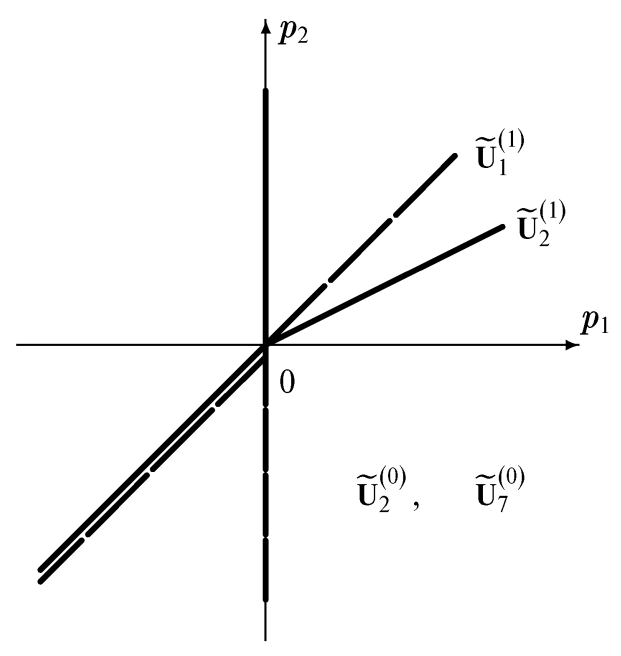

Figure 3. The superimposed normal cones of the projections of the supports of the equations of system (1.5). 
for $\widetilde{P}=(2,1)$ and $P=(2,1,2,0)$. In the sixth column $(\mathrm{T})$ the sign "+" marks the maximal values of $\left\langle\widetilde{P}, \widetilde{Q}_{k}\right\rangle$ for given $i$; the corresponding terms of the sum $f_{i}$ are included into the truncation $\widehat{f}_{i 1}^{\left(d_{i}\right)}$ in $(2.8),(2.9)$.

In the notation of $\S 1$ of Chapter I, we have $l=2$ and the resulting vector is $P=$ $\left(P^{\prime}, P^{\prime \prime}\right)$, that is, $P^{\prime}=(2,1)$. Furthermore, the vector $B_{1}^{\prime}=(-1,2)$ forms a basis in the space of vectors $Q^{\prime}=\left(q_{1}, q_{2}\right)$ satisfying the condition $\left\langle P^{\prime}, Q^{\prime}\right\rangle=0$. Applying Theorem 3 , Chapter I, $T_{3}^{\prime}=(1,0)$ and $T_{4}^{\prime}=(0,0)$, and the self-similar coordinates $\xi, h, p$ have the form

$$
\xi=\frac{r^{2}}{x}, \quad x_{3} \stackrel{\text { def }}{=} \psi=x h(\xi), \quad x_{4} \stackrel{\text { def }}{=} p=p(\xi),
$$

which corresponds to (2.10).

\section{ONE-LAYER SELF-SIMILAR ASYMPtOTICS OF THE SOLUtion}

In the self-similar coordinates (2.10) the system of equations $(2.8),(2.9)$ takes the form

$$
\begin{aligned}
& \widehat{f}_{11} \stackrel{\text { def }}{=} \frac{1}{r}\left(\frac{1}{\rho} \xi p^{\prime}\right)=0, \\
& \widehat{f}_{21} \stackrel{\text { def }}{=}-\frac{4}{x}\left(h h^{\prime \prime}+2 \xi h^{\prime \prime \prime}+2 h^{\prime \prime}+\frac{1}{\rho} \xi p^{\prime}\right)=0,
\end{aligned}
$$

where $^{\prime}=d / d \xi$. It follows from equation (3.1) that

$$
\frac{1}{\rho} \xi p^{\prime}=0
$$

consequently, from equation (3.2) we obtain

$$
\varphi(\xi, h) \stackrel{\text { def }}{=} h h^{\prime \prime}+2 \xi h^{\prime \prime \prime}+2 h^{\prime \prime}=0 .
$$

For the system (3.1), (3.2) obtained above, the boundary conditions (2.4), (2.5) in the self-similar coordinates $(2.10)$ have the form

$$
\begin{gathered}
h=\xi, \quad p=p_{0} \quad \text { as } \xi \rightarrow+\infty, \\
h=h^{\prime}=0 \quad \text { for } \xi=0 .
\end{gathered}
$$

It follows from (3.1) and (3.4) that

$$
p=\text { const }=p_{0} \quad \text { for } \xi \neq 0 .
$$

Thus, we have obtained equation (3.3) with the boundary conditions (3.5) and

$$
h=\xi \quad \text { as } \xi \rightarrow+\infty .
$$

Thus, we have proved the following.

Lemma 3. In the self-similar coordinates (2.10), the problem (2.4)-(2.7) reduces to the problem (3.1), (3.2), (3.4), (3.5), which, after elimination of $p$, reduces to the problem (3.3), (3.5), (3.6).

Lemma 4. Equation (3.3) has no solutions satisfying the boundary condition (3.5).

Proof. The support of equation (3.3) consists of the two points $Q_{1}=(-2,2)$ and $Q_{2}=$ $(-2,1)$. The support, its convex hull, and the normal cones of its faces are shown in Figure 4. The boundary condition (3.5) is imposed as $\xi \rightarrow 0$, that is, $p_{1} \leq 0$. It is clear from the boundary condition (3.5) that we are seeking solutions such that $h \rightarrow 0$ as $\xi \rightarrow 0$, that is, $p_{2} \leq 0$. We express $h$ and $\xi$ in the form (1.2) of Chapter I, so that $\xi=b_{1} \tau^{p_{1}}$ and $h=b_{2} \tau^{p_{2}}$, or $h=b_{3} \xi^{p_{2} / p_{1}}$, where $b_{1}, b_{2}$ and $b_{3}$ are constants; then $h^{\prime}=b_{4} \xi^{-1+p_{2} / p_{1}}$, 


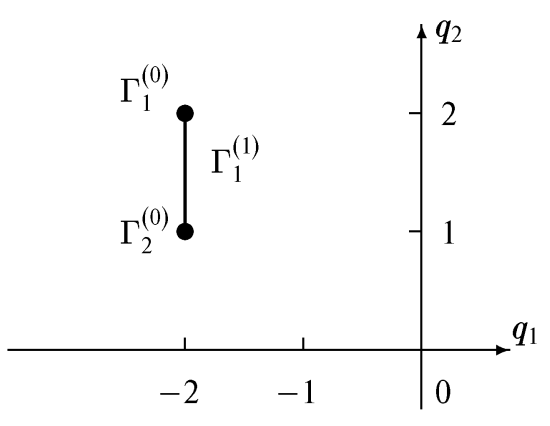

a)

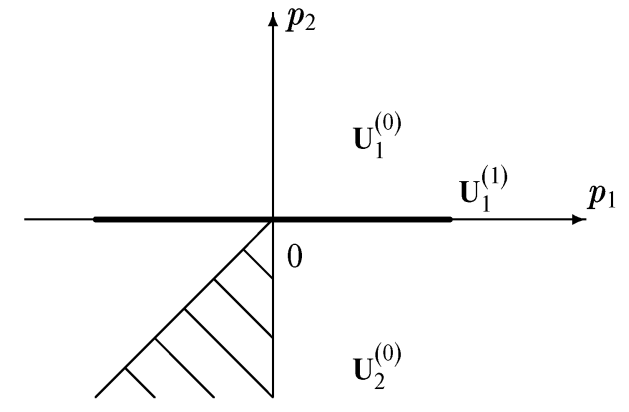

b)

FiguRE 4. (a) The support of equation (3.3) and its convex hull; (b) the normal cones of its faces.

where $b_{4}$ is a constant. By the boundary condition (3.5) we have $h^{\prime} \rightarrow 0$ as $\xi \rightarrow 0$, that is, $-1+p_{2} / p_{1} \geq 0$; consequently, $p_{2} \leq p_{1}$. Thus, we have obtained the cone for the problem $\mathcal{K}=\left\{p_{2} \leq p_{1} \leq 0\right\}$. It is shaded in Figure 4(b), which makes it clear that the cone for the problem intersects only the normal cone $\mathbf{U}_{2}^{(0)}$, which corresponds to the vertex $Q_{2}=\Gamma_{2}^{(0)}$.

The truncated equation

$$
\widehat{\varphi}_{2}^{(0)} \stackrel{\text { def }}{=} 2 h^{\prime \prime \prime} \xi+2 h^{\prime \prime}=0
$$

corresponds to the vertex $Q_{2}=\Gamma_{2}^{(0)}$. After multiplying by $\xi^{2}$, it becomes an Euler equation with characteristic equation

$$
\lambda(\lambda-1)^{2}=0
$$

its roots are $\lambda_{1}=0$ and $\lambda_{2}=1$ (a repeated root). Consequently, all the solutions of equation (3.7) have the form $h=c_{0}+c_{1} \xi+c_{2} \xi \ln \xi$, that is, $h^{\prime}=c_{1}+c_{2}+c_{2} \ln \xi$, where $c_{0}, c_{1}, c_{2}$ are arbitrary constants. From condition $(3.5)$ on $h^{\prime}$ we obtain that $c_{2}=c_{1}=0$, and from the condition on $h$ we obtain that $c_{0}=0$. Consequently, $h \equiv 0$. Thus, the truncated equation corresponding to the vertex $Q_{2}$ has no non-trivial solutions satisfying the boundary condition (3.5). According to Theorem 1, Chapter I, equation (3.3) has no non-trivial asymptotics of solutions near the needle.

Consequently, equation (3.3) has no non-trivial solutions satisfying the boundary condition (3.5).

Thus, the problem (2.4), (2.5), (2.8), (2.9) has no self-similar solutions. This result is contained in the paper of Glauert and Lighthill [11] (see also [39, §35]).

\section{ONE-LAYER NON-SELF-SIMILAR ASYMPTOTICS}

In equations $(2.8),(2.9)$ we perform the change of variables

$$
x=x, \quad \xi=\frac{r^{2}}{x}, \quad h(x, \xi)=\frac{\psi}{x}, \quad p(x, \xi)=p,
$$


that is, we take $x$ and $\xi$ for independent variables. Then

$$
\begin{gathered}
\widehat{f_{11}} \stackrel{\text { def }}{=} \frac{1}{\rho r} \xi \frac{\partial p}{\partial \xi}=0 \\
\widehat{f}_{21} \stackrel{\text { def }}{=} 4 \frac{\partial^{2} h}{\partial x \partial \xi} \frac{\partial h}{\partial \xi}-4 \frac{1}{x} h \frac{\partial^{2} h}{\partial \xi^{2}}-4 \frac{\partial h}{\partial x} \frac{\partial^{2} h}{\partial \xi^{2}}-8 \frac{1}{x} \xi \frac{\partial^{3} h}{\partial \xi^{3}} \\
-8 \frac{1}{x} \frac{\partial^{2} h}{\partial \xi^{2}}+\frac{1}{\rho}\left(\frac{\partial p}{\partial x}-\frac{1}{x} \xi \frac{\partial p}{\partial \xi}\right)=0 .
\end{gathered}
$$

The boundary conditions $(2.4),(2.5)$ take the form

$$
\begin{aligned}
& h=\frac{\partial h}{\partial \xi}=0 \text { for } \quad x \geq 0, \quad \xi=0, \\
& h=\xi, \quad p=p_{0} \quad \text { for } \quad \xi=\infty, \quad p_{0}=\text { const. }
\end{aligned}
$$

Lemma 5. The system of equations (4.2), (4.3) has no solutions satisfying the boundary conditions (4.4), (4.5).

Proof. From the boundary condition (4.5) we have $p=p_{0}$, that is, $\partial p / \partial x=0$ as $\xi \rightarrow \infty$. It follows from equation (4.2) that $\partial p / \partial \xi=0$, that is, the pressure $p$ is independent of $\xi$. Consequently, the property $\partial p / \partial x=0$ can be extended to $\xi \in(0, \infty)$. After factoring out 4 , equation (4.3), takes the form

$$
\frac{\partial^{2} h}{\partial x \partial \xi} \frac{\partial h}{\partial \xi}-\frac{1}{x} h \frac{\partial^{2} h}{\partial \xi^{2}}-\frac{\partial h}{\partial x} \frac{\partial^{2} h}{\partial \xi^{2}}-2 \frac{1}{x} \xi \frac{\partial^{3} h}{\partial \xi^{3}}-2 \frac{1}{x} \frac{\partial^{2} h}{\partial \xi^{2}}=0 .
$$

The support of equation (4.6) consists of the two points $Q_{1}=(-1,-2,2)$ and $Q_{2}=$ $(-1,-2,1)$, which have the same $q_{1}$-coordinate. Consequently [13, Ch. VI, $\S 3$, we perform the logarithmic transformation $t=\ln x$. Then

$$
\frac{\partial h}{\partial x}=x^{-1} \frac{\partial h}{\partial t}, \quad \frac{\partial^{2} h}{\partial x \partial \xi}=x^{-1} \frac{\partial^{2} h}{\partial t \partial \xi},
$$

and equation (4.6) takes the form

$$
\varphi(t, \xi, h) \stackrel{\text { def }}{=} \frac{\partial^{2} h}{\partial t \partial \xi} \frac{\partial h}{\partial \xi}-h \frac{\partial^{2} h}{\partial \xi^{2}}-\frac{\partial h}{\partial t} \frac{\partial^{2} h}{\partial \xi^{2}}-2 \xi \frac{\partial^{3} h}{\partial \xi^{3}}-2 \frac{\partial^{2} h}{\partial \xi^{2}}=0 .
$$

The support of this equation consists of the three points $Q_{1}^{\prime}=(-1,-2,2), Q_{2}^{\prime}=$ $(0,-2,2), Q_{3}^{\prime}=(0,-2,1)$. We have $x \rightarrow \infty$; therefore $t \rightarrow \infty$, that is, $p_{1}>0$. The equality $\left\langle P, Q_{1}^{\prime}\right\rangle=\left\langle P, Q_{2}^{\prime}\right\rangle-p_{1}$ implies that $\left.\left\langle P, Q_{2}^{\prime}\right\rangle\right\rangle\left\langle P, Q_{1}^{\prime}\right\rangle$. According to Chapter I, $\S 1$, the equation

$$
-\widehat{\varphi}(\xi, h) \stackrel{\text { def }}{=} h \frac{\partial^{2} h}{\partial \xi^{2}}+2 \xi \frac{\partial^{3} h}{\partial \xi^{3}}+2 \frac{\partial^{2} h}{\partial \xi^{2}}=0
$$

is a first approximation of equation (4.7) as $t \rightarrow \infty$. For fixed $x \geq 0$, equation (4.8) and the boundary condition (4.4) imply equation (3.3) with the boundary condition (3.5). By Lemma 4, equation (3.3) has no solutions satisfying the adhesion boundary condition at the needle (3.5). Consequently, equation (4.8) has no solutions satisfying the boundary condition (4.4). According to Theorem 1, Chapter I, equation (4.7) also has no such solutions. The same applies to equation (4.6) and the system (4.2), (4.3).

Thus, Lemmas 4 and 5 imply the following.

Theorem 1. Problem (2.4), (2.5), (2.8), (2.9) has no solutions. 


\section{TWO-LAYER SELF-SIMILAR ASYMPTOTICS}

We consider the possibility that a two-layer solution of the system (1.5), satisfying the boundary conditions (2.4) and (2.5) exists. The boundary conditions at infinity (2.5) give the projection of the supports of the system (1.5) that was described in the proof of Lemma 2 and shown in Figure 2. But in this case, to obtain the truncated system corresponding to the outer layer, we consider the extended cone of the system $\check{\mathbf{U}}_{J}^{D}=$ $\check{\mathbf{U}}_{1}^{(1)} \cap \check{\mathbf{U}}_{5}^{(0)}$ (Figure 3). The vector $\widetilde{P}=(1,1)$ is a direction vector of the normal cone $\widetilde{\mathbf{U}}_{1}^{(1)}$. Using $(2.6)$ we can reconstruct the vector $P=(1,1,2,0)$ from $\widetilde{P}$. The faces of the supports $\mathbf{S}\left(f_{1}\right)$ and $\mathbf{S}\left(f_{2}\right)$ correspond to the resulting vector $P$ and contain the points $Q_{1}, Q_{2}, Q_{5}$ and $Q_{6}$. Corresponding to these points we have the truncated system

$$
\begin{aligned}
& \widehat{f}_{12} \stackrel{\text { def }}{=}\left[\frac{1}{r} \frac{\partial \psi}{\partial x} \frac{\partial}{\partial r}\left(\frac{1}{r} \frac{\partial \psi}{\partial x}\right)-\frac{1}{r} \frac{\partial \psi}{\partial r} \frac{\partial}{\partial x}\left(\frac{1}{r} \frac{\partial \psi}{\partial x}\right)\right]+\frac{1}{\rho} \frac{\partial p}{\partial r}=0, \\
& \widehat{f}_{22} \stackrel{\text { def }}{=}\left[-\frac{1}{r} \frac{\partial \psi}{\partial x} \frac{\partial}{\partial r}\left(\frac{1}{r} \frac{\partial \psi}{\partial r}\right)+\frac{1}{r} \frac{\partial \psi}{\partial r} \frac{\partial}{\partial x}\left(\frac{1}{r} \frac{\partial \psi}{\partial r}\right)\right]+\frac{1}{\rho} \frac{\partial p}{\partial x}=0 .
\end{aligned}
$$

In the notation of $\S 1$ in Chapter I, we have $P=\left(P^{\prime}, P^{\prime \prime}\right)$, that is, $P^{\prime}=(1,1)$, and $B_{1}^{\prime}=(-2,2)$. According to Theorem 3, Chapter I, $T_{3}^{\prime}=(2,0), T_{4}^{\prime}=(0,0)$, and the self-similar coordinates for the problem (5.1), (2.4), (2.5) take the form

$$
\eta=\frac{r^{2}}{x^{2}}, \quad x_{3} \stackrel{\text { def }}{=} \psi=x^{2} g(\eta), \quad x_{4} \stackrel{\text { def }}{=} p=p(\eta) .
$$

In these self-similar coordinates, the system (5.1) becomes the system of ordinary differential equations

$$
\begin{aligned}
& \widehat{f}_{12} \stackrel{\text { def }}{=} \frac{2}{r}\left(-2 \eta^{-1} g^{2}+2 g g^{\prime}-4 \eta g g^{\prime \prime}+\frac{1}{\rho} \eta p^{\prime}\right)=0, \\
& \widehat{f}_{22} \stackrel{\text { def }}{=} \frac{2}{x}\left(-4 g g^{\prime \prime}-\frac{1}{\rho} \eta p^{\prime}\right)=0 .
\end{aligned}
$$

Equation (5.4) implies the equality

$$
\frac{1}{\rho} \eta p^{\prime}=-4 g g^{\prime \prime}
$$

By substituting this equality into equation (5.3) and factoring out $4 g / r$ we obtain the linear homogeneous equation

$$
\varphi(\eta, g) \stackrel{\text { def }}{=}-\eta^{-1} g+g^{\prime}-2 \eta g^{\prime \prime}-2 g^{\prime \prime}=0 .
$$

All the solutions of equation (5.6) are linear combinations $g=b_{1} g_{1}+b_{2} g_{2}$ of the two solutions

$$
g_{1}(\eta)=\sqrt{1+\eta}-\frac{\eta}{2} \ln \frac{\sqrt{1+\eta}-1}{\sqrt{1+\eta}+1}, \quad g_{2}=\eta,
$$

where $b_{1}$ and $b_{2}$ are constants. Equation (5.6) is similar to equation 2.267a in Kamke's handbook [40, although it does not reduce to it. Their solutions are also similar. It is easy to see that $g_{1}(\eta) \sim 2 \sqrt{\eta}$ as $\eta \rightarrow \infty$, while for small $|\eta|$ we have

$$
g_{1}(\eta)=1-\frac{\eta}{2} \ln \eta+\frac{1+\ln 4}{2} \eta+\sum_{k=2}^{\infty} c_{k} \eta^{k}, \text { where } c_{k}=\text { const. }
$$


The boundary conditions at infinity (2.5) in the self-similar coordinates (5.2) take the form

$$
\frac{\partial g}{\partial \eta}=1, \quad p=p_{0}=\mathrm{const} \quad \text { as } \eta \rightarrow \infty .
$$

The first condition is satisfied only by solutions $g=b_{1} g_{1}+b_{2} g_{2}$ with $b_{2}=1$, that is, by solutions of the form

$$
g=b_{1} g_{1}(\eta)+\eta
$$

Consequently, as $\eta \rightarrow 0$, these solutions have asymptotics of the two forms

$$
\begin{aligned}
& \text { a) } g \sim b_{1}=\text { const if } b_{1} \neq 0, \\
& \text { b) } g=\eta \quad \text { if } b_{1}=0 .
\end{aligned}
$$

In case a), according to (5.5) and (5.7), the pressure satisfies $p \sim-2 \rho b_{1}^{2} / \eta$ as $\eta \rightarrow 0$, so that $p \rightarrow-\infty$ as $\eta \rightarrow 0$. But such a solution has no physical meaning.

In case b), according to (5.5), the pressure satisfies $p=p_{0}=$ const for all $\eta$, including for $\eta \rightarrow 0$. In this case we have

$$
\psi=r^{2}, \quad p=p_{0}=\text { const }
$$

in the outer layer, and these are boundary conditions for the inner layer as $\xi \rightarrow \infty$. But this case was considered in $\S 3$, where it was shown that there are no solutions satisfying the adhesion condition at the needle.

Thus, we have proved the following.

Lemma 6. The problem (1.5), (2.4), (2.5) has no two-layer self-similar solutions.

\section{TWO-LAYER NON-SELF-SIMILAR ASYMPTOTICS}

As a result of the change of variables

$$
x=x, \quad \eta=\frac{r^{2}}{x^{2}}, \quad g(x, \eta)=\frac{\psi}{x^{2}}, \quad p(x, \eta)=p
$$

the system (5.1) takes the form

$$
\begin{aligned}
& \widehat{f}_{12} \stackrel{\text { def }}{=} \frac{2}{r}\left[\left(-2 \eta^{-1} g^{2}-2 x \eta^{-1} g \frac{\partial g}{\partial x}-\frac{1}{2} x^{2} \eta^{-1}\left(\frac{\partial g}{\partial x}\right)^{2}\right.\right. \\
& +2 g \frac{\partial g}{\partial \eta}+2 x g \frac{\partial^{2} g}{\partial x \partial \eta}+x^{2} \frac{\partial g}{\partial x} \frac{\partial^{2} g}{\partial x \partial \eta}-4 \eta g \frac{\partial^{2} g}{\partial \eta^{2}} \\
& \left.\left.-2 x \eta \frac{\partial g}{\partial x} \frac{\partial^{2} g}{\partial \eta^{2}}-2 x \frac{\partial g}{\partial x} \frac{\partial g}{\partial \eta}+2 x \eta \frac{\partial g}{\partial \eta} \frac{\partial^{2} g}{\partial x \partial \eta}\right)+\frac{1}{\rho} \eta \frac{\partial p}{\partial \eta}\right]=0, \\
& \widehat{f}_{22} \stackrel{\text { def }}{=} \frac{2}{x}\left[-4 g \frac{\partial^{2} g}{\partial \eta^{2}}+\left(2 x \frac{\partial g}{\partial \eta} \frac{\partial^{2} g}{\partial x \partial \eta}-x \frac{\partial g}{\partial x} \frac{\partial^{2} g}{\partial \eta^{2}}\right)+\frac{x}{2 \rho} \frac{\partial p}{\partial x}-\frac{1}{\rho} \eta \frac{\partial p}{\partial \eta}\right]=0 .
\end{aligned}
$$

The boundary conditions (2.4), (2.5) take the form

$$
\begin{gathered}
g=\frac{\partial g}{\partial \eta}=0 \quad \text { for } x \geq 0, \eta=0, \\
g=\eta, \quad p=p_{0} \quad \text { for } \eta=\infty, \quad \text { where } p_{0}=\text { const. }
\end{gathered}
$$

In equations (6.2) and (6.3), the summands in parentheses have vectorial power exponents $Q=\left(q_{1}, q_{2}, q_{3}, q_{4}\right)$ with $q_{1}=0$ and $q_{1}=-1$, respectively. We perform the logarithmic transformation

$$
t=\ln x
$$


then $\partial g / \partial x=x^{-1} \partial g / \partial t$. We obtain

$$
\begin{aligned}
& \frac{r}{2} \widehat{f}_{12} \stackrel{\text { def }}{=}\left(2 g \frac{\partial g}{\partial \eta}-2 \eta^{-1} g^{2}-4 \eta g \frac{\partial^{2} g}{\partial \eta^{2}}\right) \\
&+\left(2 g \frac{\partial^{2} g}{\partial t \partial \eta}-2 \eta^{-1} g \frac{\partial g}{\partial t}-2 \eta \frac{\partial g}{\partial t} \frac{\partial^{2} g}{\partial \eta^{2}}-2 \frac{\partial g}{\partial t} \frac{\partial g}{\partial \eta}+2 \eta \frac{\partial g}{\partial \eta} \frac{\partial^{2} g}{\partial t \partial \eta}\right) \\
&+\left(\frac{\partial g}{\partial t} \frac{\partial^{2} g}{\partial t \partial \eta}-\frac{1}{2} \eta^{-1}\left(\frac{\partial g}{\partial t}\right)^{2}\right)+\frac{1}{\rho} \eta \frac{\partial p}{\partial \eta}=0, \\
& \frac{x}{2} \widehat{f}_{22} \stackrel{\text { def }}{=}-4 g \frac{\partial^{2} g}{\partial \eta^{2}}+\left(2 \frac{\partial g}{\partial \eta} \frac{\partial^{2} g}{\partial t \partial \eta}-\frac{\partial g}{\partial t} \frac{\partial^{2} g}{\partial \eta^{2}}\right)+\frac{1}{2 \rho} \frac{\partial p}{\partial t}-\frac{1}{\rho} \eta \frac{\partial p}{\partial \eta}=0 .
\end{aligned}
$$

The supports of equations (6.7), (6.8) consist of the following points:

$\mathbf{S}\left(\widehat{f}_{12}\right)=\left\{Q_{1}^{\prime}=(0,-1,2,0), Q_{2}^{\prime}=(-1,-1,2,0), Q_{3}^{\prime}=(-2,-1,2,0), Q_{4}^{\prime}=(0,0,0,1)\right\} ;$

$\mathbf{S}\left(\widehat{f}_{22}\right)=\left\{Q_{5}^{\prime}=(0,-2,2,0), Q_{6}^{\prime}=(-1,-2,2,0), Q_{7}^{\prime}=(-1,0,0,1), Q_{8}^{\prime}=(0,0,0,1)\right\}$.

Now, $t=\ln x$, so $t \rightarrow \infty$ as $x \rightarrow \infty$, that is, $p_{1}>0$. Consequently,

$$
\left\langle Q_{1}^{\prime}, P\right\rangle>\left\langle Q_{2}^{\prime}, P\right\rangle>\left\langle Q_{3}^{\prime}, P\right\rangle \text {. }
$$

In the second equation, $\left\langle Q_{5}^{\prime}, P\right\rangle>\left\langle Q_{6}^{\prime}, P\right\rangle$ and $\left\langle Q_{8}^{\prime}, P\right\rangle>\left\langle Q_{7}^{\prime}, P\right\rangle$. According to $\S 1$, Chapter I, a first approximation of the system of equations (6.7), (6.8) as $t \rightarrow \infty$ is given by the truncated system that coincides exactly with the system (5.3), (5.4), while the boundary conditions (6.5) coincide with the conditions (5.8). We seek solutions of the system (6.7), (6.8) in the form of expansions in powers of $t$ :

$$
\begin{aligned}
& g(t, \eta)=g^{(0)}(\eta) t^{m}+\sum_{k=1}^{\infty} g^{(k)}(\eta) t^{m-k}, \\
& p(t, \eta)=p^{(0)}(\eta) t^{m}+\sum_{k=1}^{\infty} p^{(k)}(\eta) t^{m-k} .
\end{aligned}
$$

Then $g=g^{(0)}(\eta), p=p^{(0)}(\eta)$ is a solution of the truncated system (5.3), (5.4). Since the solutions (6.9) must satisfy the boundary conditions (6.5), which coincide with the conditions (5.8), we have $m=0$. Consequently, the solutions (6.9) of (6.7), (6.8) have the form

$$
\begin{aligned}
& g(t, \eta)=g^{(0)}(\eta)+\sum_{k=1}^{\infty} g^{(k)}(\eta) t^{-k}, \\
& p(t, \eta)=p^{(0)}(\eta)+\sum_{k=1}^{\infty} p^{(k)}(\eta) t^{-k},
\end{aligned}
$$

where $g^{(0)}(\eta), p^{(0)}(\eta)$ is a solution of the system (5.3), (5.4) satisfying the conditions (5.8). The asymptotics of the solutions (6.10) as $t \rightarrow \infty$ and $\eta \rightarrow 0$ coincide with the asymptotics for $g^{(0)}(\eta), p^{(0)}(\eta)$ as $\eta \rightarrow 0$, that is, they have the form

$$
\begin{aligned}
& \text { a) } g \sim b_{1}=\text { const, } \quad p \sim-\frac{2 \rho b_{1}^{2}}{\eta}, \\
& \text { b) } g \sim \eta, \quad p \sim p_{0}=\text { const, that is, } \quad \psi \sim r^{2} .
\end{aligned}
$$

Case a) has no physical meaning, and in case b) we obtain the one-layer situation considered in $\S 4$, where we showed that no solution satisfies all the boundary conditions.

Thus, we have proved the following assertion.

Lemma 7. The problem (1.5), (2.4), (2.5) has no two-layer non-self-similar solutions.

Lemmas 6 and 7 imply the following theorem.

Theorem 2. The problem (1.5), (2.4), (2.5) has no two-layer solutions. 


\section{Chapter III. FLOW OF A VISCOUS COMPRESSIBLE HEAT-CONDUCTING GAS PAST A NEEDLE}

\section{The System of PARTial DifFEREntial EQUATIONS}

A steady motion of a viscous heat-conducting gas is described by the following system of equations ([37, Ch. XI, § 108], [38, Ch. 3, § 3.3]):

$$
\begin{gathered}
\rho\langle\mathbf{V}, \nabla\rangle \mathbf{V}=-\operatorname{grad}\left(p+\frac{2}{3} \mu \operatorname{div} \mathbf{V}\right)+2 \operatorname{Div}(\mu \dot{\mathbf{S}}), \\
\operatorname{div}(\rho \mathbf{V})=0, \\
\rho\langle\mathbf{V}, \nabla\rangle\left(h+\frac{\mathbf{V}^{2}}{2}\right)=\operatorname{div}\left(2 \mu \mathbf{V} \dot{\mathbf{S}}-\frac{2}{3} \mu \mathbf{V} \operatorname{div} \mathbf{V}+\frac{\mu}{\sigma} \operatorname{grad} h\right),
\end{gathered}
$$

where $\nabla=(\partial / \partial x, \partial / \partial y, \partial / \partial z), \quad \mathbf{V}$ is the velocity vector, $p$ is the pressure, $\rho$ is the density, $h$ is the enthalpy, $\mu$ is the dynamical viscosity coefficient, $\sigma=\mu c_{p} / \lambda$ is the Prandtl number, $\lambda$ is the heat-transfer coefficient of the gas, $c_{p}$ is the specific heat capacity at constant pressure, $\dot{\mathbf{S}}$ is the deformation velocity tensor.

We denote by $V_{x}, V_{r}, V_{\varepsilon}$ the components of the velocity vector $\mathbf{V}$ in the cylindrical system of coordinates $(x, r, \varepsilon)$ and, setting $V_{\varepsilon}=0$, consider a flow that is symmetric with respect to the $x$-axis. In this case all the derivatives with respect to the angle $\varepsilon$ vanish, while the components of the tensor $\dot{\mathbf{S}}$ have the following form:

$$
\begin{aligned}
\dot{S}_{x x}=\frac{\partial V_{x}}{\partial x}, \quad \dot{S}_{r r}=\frac{\partial V_{r}}{\partial r}, \quad \dot{S}_{\varepsilon \varepsilon}=\frac{V_{r}}{r}, & \dot{S}_{r \varepsilon}=\dot{S}_{\varepsilon r}=0 \\
\dot{S}_{r x}=\dot{S}_{x r}=\frac{1}{2}\left(\frac{\partial V_{r}}{\partial x}+\frac{\partial V_{x}}{\partial r}\right), & \dot{S}_{\varepsilon x}=\dot{S}_{x \varepsilon}=0 .
\end{aligned}
$$

We assume that $\mu$ and $h$ are connected by the relation

$$
\mu=C^{n} h^{n} \quad(n=\mathrm{const}), \quad n \in[0,1],
$$

and the pressure $p$ is expressed in terms of $\rho$ and $h$ by the Clapeyron equation:

$$
p=A \rho h,
$$

where the constants are as follows: $C=(\gamma-1) M_{\infty}^{2}, M_{\infty}$ is the Mach number, $A=$ $(\gamma-1) / \gamma, \gamma=c_{p} / c_{v}=$ const, and $c_{v}$ is the specific heat capacity of the gas at constant volume.

We use (1.4) and (1.5) to eliminate $\mu$ and $p$ from equations (1.1) and (1.3) and use (1.2) to introduce the stream function $\psi(x, r)$ by the formulae

$$
r \rho V_{r}=-\frac{\partial \psi}{\partial x}, \quad r \rho V_{x}=\frac{\partial \psi}{\partial r} .
$$

A steady axisymmetric flow of the viscous compressible heat-conducting gas is now described by the following system of three equations [41, 42]:

$$
\begin{aligned}
f_{1} \stackrel{\text { def }}{=} & {\left[-\frac{1}{r} \frac{\partial \psi}{\partial x} \frac{\partial}{\partial r}\left(\frac{1}{\rho r} \frac{\partial \psi}{\partial x}\right)+\frac{1}{r} \frac{\partial \psi}{\partial r} \frac{\partial}{\partial x}\left(\frac{1}{\rho r} \frac{\partial \psi}{\partial x}\right)\right]-A \frac{\partial}{\partial r}(\rho h) } \\
+ & {\left[\frac{2}{3} C^{n} \frac{\partial}{\partial r}\left(\frac{h^{n}}{r} \frac{\partial}{\partial r}\left(\frac{1}{\rho} \frac{\partial \psi}{\partial x}\right)\right)-\frac{2}{3} C^{n} \frac{\partial}{\partial r}\left(\frac{h^{n}}{r} \frac{\partial}{\partial x}\left(\frac{1}{\rho} \frac{\partial \psi}{\partial r}\right)\right)\right.} \\
& -\frac{2 C^{n}}{r} \frac{\partial}{\partial r}\left(h^{n} r \frac{\partial}{\partial r}\left(\frac{1}{\rho r} \frac{\partial \psi}{\partial x}\right)\right)+C^{n} \frac{\partial}{\partial x}\left(h^{n} \frac{\partial}{\partial r}\left(\frac{1}{\rho r} \frac{\partial \psi}{\partial r}\right)\right. \\
& \left.+\frac{2 C^{n} h^{n}}{\rho r^{3}} \frac{\partial \psi}{\partial x}\right]-C^{n} \frac{\partial}{\partial x}\left(h^{n} \frac{\partial}{\partial x}\left(\frac{1}{\rho r} \frac{\partial \psi}{\partial x}\right)\right)=0
\end{aligned}
$$




$$
\begin{aligned}
f_{2} \stackrel{\text { def }}{=} & {\left[\frac{1}{r} \frac{\partial \psi}{\partial x} \frac{\partial}{\partial r}\left(\frac{1}{\rho r} \frac{\partial \psi}{\partial r}\right)-\frac{1}{r} \frac{\partial \psi}{\partial r} \frac{\partial}{\partial x}\left(\frac{1}{\rho r} \frac{\partial \psi}{\partial r}\right)\right]-A \frac{\partial}{\partial x}(\rho h) } \\
+ & {\left[\frac{2}{3} C^{n} \frac{\partial}{\partial x}\left(\frac{h^{n}}{r} \frac{\partial}{\partial r}\left(\frac{1}{\rho} \frac{\partial \psi}{\partial x}\right)\right)-\frac{2}{3} C^{n} \frac{\partial}{\partial x}\left(\frac{h^{n}}{r} \frac{\partial}{\partial x}\left(\frac{1}{\rho} \frac{\partial \psi}{\partial r}\right)\right)\right.} \\
& \left.-\frac{C^{n}}{r} \frac{\partial}{\partial r}\left(h^{n} r \frac{\partial}{\partial x}\left(\frac{1}{\rho r} \frac{\partial \psi}{\partial x}\right)\right)+2 C^{n} \frac{\partial}{\partial x}\left(h^{n} \frac{\partial}{\partial x}\left(\frac{1}{\rho r} \frac{\partial \psi}{\partial r}\right)\right)\right] \\
+ & \frac{C^{n}}{r} \frac{\partial}{\partial r}\left(h^{n} r \frac{\partial}{\partial r}\left(\frac{1}{\rho r} \frac{\partial \psi}{\partial r}\right)\right)=0,
\end{aligned}
$$

$$
\begin{aligned}
f_{3} \stackrel{\text { def }}{=} & {\left[\frac{1}{r} \frac{\partial \psi}{\partial x} \frac{\partial h}{\partial r}-\frac{1}{r} \frac{\partial \psi}{\partial r} \frac{\partial h}{\partial x}-\frac{A}{\rho r} \frac{\partial \psi}{\partial x} \frac{\partial(\rho h)}{\partial r}+\frac{A}{\rho r} \frac{\partial \psi}{\partial r} \frac{\partial(\rho h)}{\partial x}\right] } \\
& +C^{n} h^{n}\left(\frac{\partial}{\partial x}\left(\frac{1}{\rho r} \frac{\partial \psi}{\partial x}\right)\right)^{2}+\left[2 C^{n} h^{n}\left(\frac{\partial}{\partial r}\left(\frac{1}{\rho r} \frac{\partial \psi}{\partial x}\right)\right)^{2}\right. \\
& +2 C^{n} h^{n}\left(\frac{1}{r^{2} \rho} \frac{\partial \psi}{\partial x}\right)^{2}+2 C^{n} h^{n}\left(\frac{\partial}{\partial x}\left(\frac{1}{\rho r} \frac{\partial \psi}{\partial r}\right)\right)^{2} \\
& -C^{n} h^{n} \frac{\partial}{\partial x}\left(\frac{1}{\rho r} \frac{\partial \psi}{\partial x}\right) \frac{\partial}{\partial r}\left(\frac{1}{\rho r} \frac{\partial \psi}{\partial r}\right)-\frac{2}{3} C^{n} h^{n}\left(\frac{1}{r} \frac{\partial}{\partial r}\left(\frac{1}{\rho} \frac{\partial \psi}{\partial x}\right)\right)^{2} \\
& \left.+\frac{4 C^{n} h^{n}}{3 r} \frac{\partial}{\partial r}\left(\frac{1}{\rho} \frac{\partial \psi}{\partial x}\right) \frac{\partial}{\partial x}\left(\frac{1}{\rho r} \frac{\partial \psi}{\partial r}\right)-\frac{2}{3} C^{n} h^{n}\left(\frac{\partial}{\partial x}\left(\frac{1}{\rho r} \frac{\partial \psi}{\partial r}\right)\right)^{2}\right] \\
& +C^{n} h^{n}\left(\frac{\partial}{\partial r}\left(\frac{1}{\rho r} \frac{\partial \psi}{\partial r}\right)\right)^{2}+\frac{C^{n}}{\sigma r} \frac{\partial}{\partial r}\left(r h^{n} \frac{\partial h}{\partial r}\right)+\frac{C^{n}}{\sigma} \frac{\partial}{\partial x}\left(h^{n} \frac{\partial h}{\partial x}\right)=0,
\end{aligned}
$$

where the $x$-coordinate is directed along the axis of symmetry and $r$ is the distance from the $x$-axis (these are independent variables); the dependent variables are the stream function $\psi$, the density $\rho$ and the enthalpy $h$; and $A, C, \sigma>0, n \in[0,1]$ are constants. We have $r \geq 0, h>0$, and

$$
C^{n}>0, \quad h^{n}>0 .
$$

Here the stream function is $\psi=\psi_{I I I}=\rho \psi_{I I}$, where $\psi_{I I}$ is the stream function $\psi$ of Chapter II. When $h, \rho$ and $\nu=C^{n} h^{n}$ are constant, if we reject the Clapeyron equation (1.5), equations (1.7) and (1.8) turn into the equations $f_{1}=0$ and $f_{2}=0$, respectively, in the system (1.5) from Chapter II. However, in this case equation (1.9) does not turn into an identical equality. This is connected with the fact that the system (1.7)-(1.9) corresponds to a gas, while the system (1.5) from Chapter II, to a fluid. Therefore the system (1.7)-(1.9) cannot be regarded as a perturbation of system (1.5) in Chapter II.

The needle is given as $x \geq 0, r=0$. Here, in the notation of Chapter $1, \S 1$,

$$
x_{1}=x, \quad x_{2}=r, \quad x_{3}=\psi, \quad x_{4}=\rho, \quad x_{5}=h .
$$

The boundary conditions are given at infinity and at the needle (cf. formulae (2.2), (2.3) in Chapter II):

$$
\begin{gathered}
\psi=\psi_{0} r^{2}, \quad \rho=\rho_{0}, \quad h=h_{0} \quad \text { at } x=-\infty, \quad \text { where } \psi_{0}, \rho_{0}, h_{0}=\text { const } \neq 0, \\
\frac{\partial \psi}{\partial x}=\frac{\partial \psi}{\partial r}=\frac{\partial^{2} \psi}{\partial x \partial r}=\frac{\partial^{2} \psi}{\partial r^{2}}=0 \quad \text { for } x \geq 0, \quad r=0 .
\end{gathered}
$$

The conditions (1.13) were derived in Chapter II, $\S 2$.

Lemma 1. For the problem (1.7)-(1.9), (1.12), (1.13) the boundary conditions are

$$
\psi=\psi_{0} r^{2}, \quad \rho=\rho_{0}, \quad h=h_{0} \quad \text { at } r=+\infty .
$$


Proof. This lemma is similar to Chapter II, Lemma 1 and is proved in exactly the same way. We observe that the functions

$$
\psi=\psi_{0} r^{2}, \quad \rho=\rho_{0}, \quad h=h_{0} ; \quad \psi_{0}, \rho_{0}, h_{0}=\text { const },
$$

annihilate each of the terms in equations (1.7)-(1.9). Consequently, the functions (1.15) give a solution of any truncated system for the system (1.7)-(1.9). Therefore for $x<0$ and $r \rightarrow \infty$ the expressions (1.15) are boundary conditions, which extend the boundary conditions (1.12). Moreover, they can also be extended for $x>0, r \rightarrow \infty$.

Therefore in what follows we seek a solution of the boundary problem (1.7)-(1.9), (1.13), (1.14).

Lemma 2. For the system (1.7)-(1.9), the truncated system corresponding to the boundary layer on the needle as $x \rightarrow+\infty$ and to the boundary conditions (1.14) is unique. This truncated system has normal vector $P=(2,1,2,0,0)$ and has the form

$$
\begin{aligned}
& \widehat{f}_{12}^{(0)} \stackrel{\text { def }}{=}-A \frac{\partial(\rho h)}{\partial r}=0, \\
& \widehat{f}_{22}^{(2)} \stackrel{\text { def }}{=}\left[\frac{1}{r} \frac{\partial \psi}{\partial x} \frac{\partial}{\partial r}\left(\frac{1}{\rho r} \frac{\partial \psi}{\partial r}\right)-\frac{1}{r} \frac{\partial \psi}{\partial r} \frac{\partial}{\partial x}\left(\frac{1}{\rho r} \frac{\partial \psi}{\partial r}\right)\right]-A \frac{\partial}{\partial x}(\rho h) \\
& +\frac{C^{n}}{r} \frac{\partial}{\partial r}\left(h^{n} r \frac{\partial}{\partial r}\left(\frac{1}{\rho r} \frac{\partial \psi}{\partial r}\right)\right)=0 \\
& \widehat{f}_{32}^{(2)} \stackrel{\text { def }}{=}\left[\frac{1}{r} \frac{\partial \psi}{\partial x} \frac{\partial h}{\partial r}-\frac{1}{r} \frac{\partial \psi}{\partial r} \frac{\partial h}{\partial x}-\frac{A}{\rho r} \frac{\partial \psi}{\partial x} \frac{\partial(\rho h)}{\partial r}+\frac{A}{\rho r} \frac{\partial \psi}{\partial r} \frac{\partial(\rho h)}{\partial x}\right] \\
& +C^{n} h^{n}\left(\frac{\partial}{\partial r}\left(\frac{1}{\rho r} \frac{\partial \psi}{\partial r}\right)\right)^{2}+\frac{C^{n}}{\sigma r} \frac{\partial}{\partial r}\left(r h^{n} \frac{\partial h}{\partial r}\right)=0,
\end{aligned}
$$

and the self-similar coordinates $\xi, G, \mathrm{P}, H$ for the problem (1.13), (1.14), (1.16)-(1.18) are

$$
\xi=\frac{r^{2}}{x}, \quad \psi=x G(\xi), \quad \rho=\mathrm{P}(\xi), \quad h=H(\xi) .
$$

Proof. The supports of equations (1.7)-(1.9) are presented in Table 2. The first column contains the number $i$ of the equation $f_{i}=0$, the second column contains the number $k$ of the point $Q_{k}$ of the support, and the third column contains the vectors (or points) $Q_{k}$. In equations (1.7)-(1.9), terms with the same vectorial power exponent are grouped in the square brackets.

According to (1.14), as $r \rightarrow+\infty$ there are three boundary conditions having the same form as (1.16) in Chapter I:

$$
f_{4} \stackrel{\text { def }}{=} \psi-\psi_{0} r^{2}=0, \quad f_{5} \stackrel{\text { def }}{=} \rho-\rho_{0}=0, \quad f_{6} \stackrel{\text { def }}{=} h-h_{0}=0 .
$$

To each of them corresponds a support consisting of two points: namely, $\mathbf{S}\left(f_{4}\right)$ consists of the points $Q_{15}=(0,0,1,0,0)$ and $Q_{16}=(0,2,0,0,0) ; \mathbf{S}\left(f_{5}\right)$ consists of the points $Q_{17}=(0,0,0,1,0)$ and $Q_{18}=(0,0,0,0,0)$; and $\mathbf{S}\left(f_{6}\right)$ consists of the points $Q_{19}=$ $(0,0,0,0,1)$ and $Q_{20}=(0,0,0,0,0)$. According to Theorem 2, Chapter I, the vector $P$ must satisfy conditions (1.17) in Chapter I: namely, $\left\langle Q_{15}, P\right\rangle=\left\langle Q_{16}, P\right\rangle$, that is, $p_{3}=2 p_{2} ;\left\langle Q_{17}, P\right\rangle=\left\langle Q_{18}, P\right\rangle$, that is, $p_{4}=0$; and $\left\langle Q_{19}, P\right\rangle=\left\langle Q_{20}, P\right\rangle$, that is, $p_{5}=0$. Thus, three conditions on the vector $P=\left(p_{1}, p_{2}, p_{3}, p_{4}, p_{5}\right)$ have been obtained:

$$
p_{3}=2 p_{2}, \quad p_{4}=p_{5}=0 .
$$


TABLE 2. Selection of the truncated system. Here, $\Delta_{k} \stackrel{\text { def }}{=}\left\langle\widetilde{P}, \widetilde{Q}_{k}\right\rangle=\left\langle P, Q_{k}\right\rangle$.

\begin{tabular}{|c|c|c|c|c|c|}
\hline$i$ & $k$ & $Q_{k}$ & $\widetilde{Q}_{k}$ & $\Delta_{k}$ & $\mathrm{~T}$ \\
\hline \multirow[t]{4}{*}{1} & 1 & $-2,-3,2,-1,0$ & $-2, \quad 1$ & -3 & \\
\hline & 2 & $0,-1,0, \quad 1,1$ & $0,-1$ & -1 & + \\
\hline & 3 & $-1,-3,1,-1, n$ & $-1,-1$ & -3 & \\
\hline & 4 & $-3,-1,1,-1, n$ & $-3, \quad 1$ & -5 & \\
\hline \multirow[t]{4}{*}{2} & 5 & $-1,-4,2,-1,0$ & $-1, \quad 0$ & -2 & + \\
\hline & 6 & $-1, \quad 0,0, \quad 1,1$ & $-1, \quad 0$ & -2 & + \\
\hline & 7 & $-2,-2,1,-1, n$ & $-2, \quad 0$ & -4 & \\
\hline & 8 & $0,-4,1,-1, n$ & $0,-2$ & -2 & + \\
\hline \multirow[t]{6}{*}{3} & 9 & $-1,-2,1, \quad 0,1$ & $-1, \quad 0$ & -2 & + \\
\hline & 10 & $-4,-2,2,-2, n$ & $-4, \quad 2$ & -6 & \\
\hline & 11 & $-2,-4,2,-2, n$ & $-2, \quad 0$ & -4 & \\
\hline & 12 & $0,-6,2,-2, n$ & $0,-2$ & -2 & + \\
\hline & 13 & $0,-2,0, \quad 0, n+1$ & $0,-2$ & -2 & + \\
\hline & 14 & $-2, \quad 0,0, \quad 0, n+1$ & $-2, \quad 0$ & -4 & \\
\hline
\end{tabular}

In the notation (1.17) and (1.18) from Chapter I, here $m^{\prime}=l=2$ and $R_{3}^{\prime}=(0,2)$, $R_{4}^{\prime}=R_{5}^{\prime}=0$. By Theorem 2, Chapter I, according to conditions (1.20) the vector $Q_{k}$ can be projected onto the two-dimensional plane $\widetilde{Q}=\left(\widetilde{q}_{1}, \widetilde{q}_{2}\right)$ using Chapter I, (1.18). Effectively, $\widetilde{q}_{1}=q_{1}$ and $\widetilde{q}_{2}=q_{2}+2 q_{3}$, while the values of $q_{4}$ and $q_{5}$ are neglected. The fourth column of Table 2 contains the projections $\widetilde{Q}_{k}=\left(q_{1}, q_{2}+2 q_{3}\right)$ of the vectors $Q_{k}$. The projections $\widetilde{\mathbf{S}}\left(f_{1}\right)-\widetilde{\mathbf{S}}\left(f_{3}\right)$ of the supports of equations (1.7)-(1.9), their convex hulls $\widetilde{\Gamma}_{1}-\widetilde{\Gamma}_{3}$, and their normal cones are presented in Figure 5 .

The needle is described as $x \geq 0, r=0$. Near the needle, as $x \rightarrow+\infty$ and $r \rightarrow 0$, we have $p_{1}>0$ and $p_{2}<0$. Consequently, the fourth quadrant of the $\left(p_{1}, p_{2}\right)$-plane corresponds to the needle. As $x \rightarrow+\infty$ and $r \rightarrow+\infty$ the boundary conditions (1.14) mean that $p_{1}, p_{2}>0$, that is, points in the first quadrant of the $\left(p_{1}, p_{2}\right)$-plane correspond to them. We are interested in the faces of the projections $\widetilde{\Gamma}_{i}, i=1,2,3$, whose extended normal cone contains both the fourth quadrant and points in the first quadrant of the $\left(p_{1}, p_{2}\right)$-plane. We superimpose the pictures of the normal cones of the projections in Figure 5(b) in a single picture in Figure 6. It is clear from this picture that the fourth quadrant and points in the first quadrant are contained only in the extended normal cone of the system $\check{\mathbf{U}}_{J}^{D}=\check{\mathbf{U}}_{5}^{(1)} \cap \check{\mathbf{U}}_{8}^{(1)}$. The vector $\widetilde{P}=(2,1)$ is a direction vector of the normal cones $\widetilde{\mathbf{U}}_{5}^{(1)}=\widetilde{\mathbf{U}}_{8}^{(1)}$. From the vector $\widetilde{P}=\left(p_{1}, p_{2}\right)$ we reconstruct the vector $P=$ $\left(p_{1}, p_{2}, p_{3}, p_{4}, p_{5}\right)$ according to conditions $(1.20)$ and obtain the vector $P=(2,1,2,0,0)$ in the original $\left(p_{1}, p_{2}, p_{3}, p_{4}, p_{5}\right)$-coordinates.

The following vertices and edges of the projections: $\widetilde{Q}_{2} ; \quad \widetilde{Q}_{5}=\widetilde{Q}_{6}, \quad \widetilde{Q}_{8}, \quad \widetilde{\Gamma}_{5}^{(1)}$; $\widetilde{Q}_{9}, \quad \widetilde{Q}_{12}=\widetilde{Q}_{13}, \quad \widetilde{\Gamma}_{8}^{(1)}$ correspond to the normal cones obtained above. In the original $\left(q_{1}, q_{2}, q_{3}, q_{4}, q_{5}\right)$-coordinates the points $Q_{2}, Q_{5}, Q_{6}, Q_{8}, Q_{9}, Q_{12}, Q_{13}$ of the supports of equations (1.7)-(1.9) correspond to them. These points have the corresponding truncated system (1.16)-(1.18). 
(1.1)
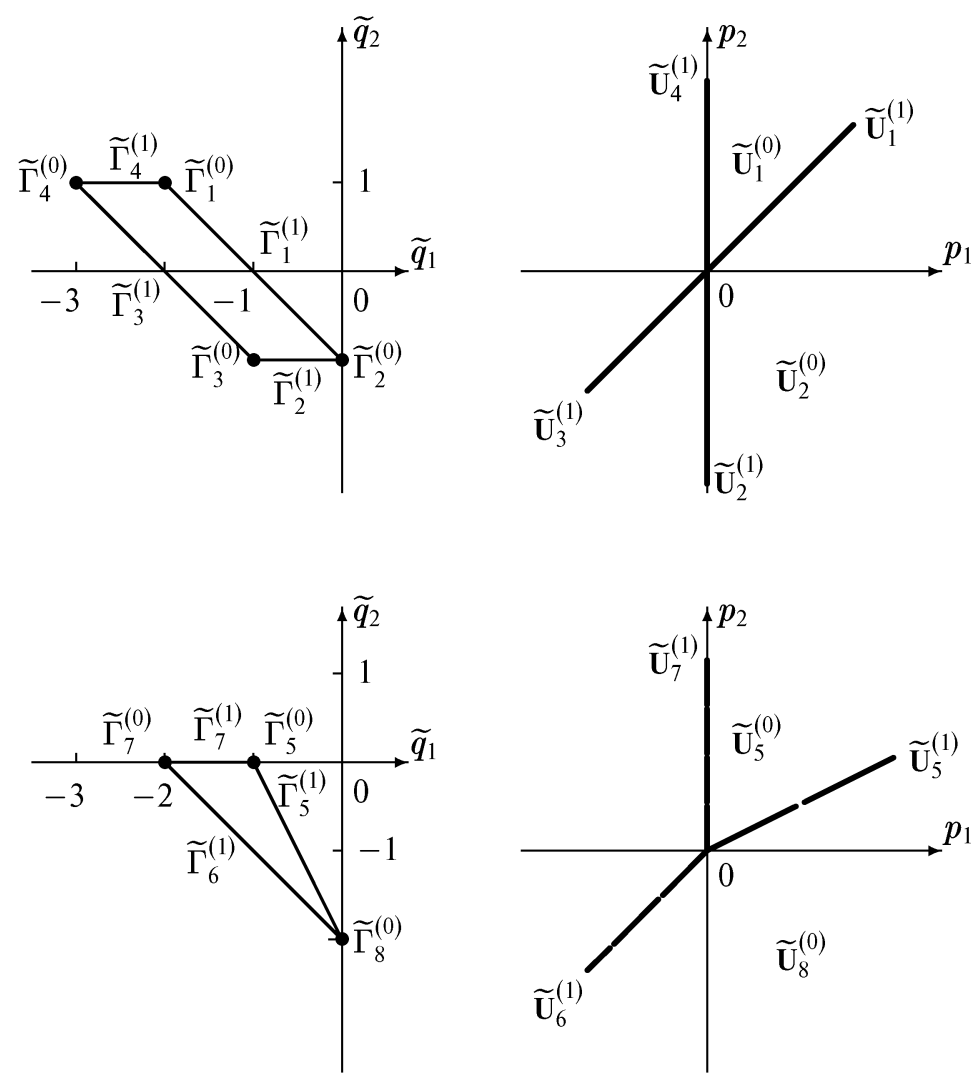

(1.3)

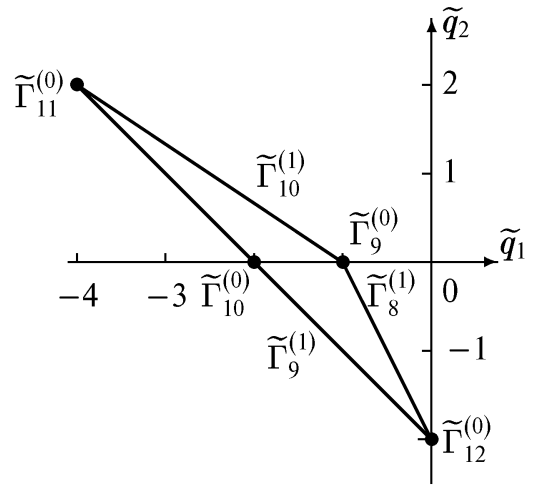

a)

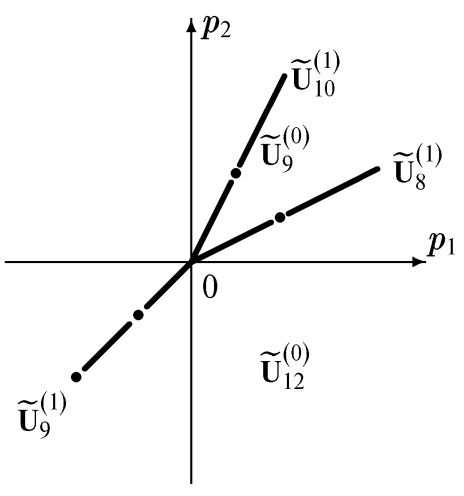

b)

FIGURE 5. (a) The projections of the supports of the equations of the system (1.7)-(1.9); (b) the normal cones of the projections.

The fifth column of Table 2 contains the values of the scalar product

$$
\Delta_{k}=\left\langle\widetilde{P}, \widetilde{Q}_{k}\right\rangle=\left\langle P, Q_{k}\right\rangle
$$




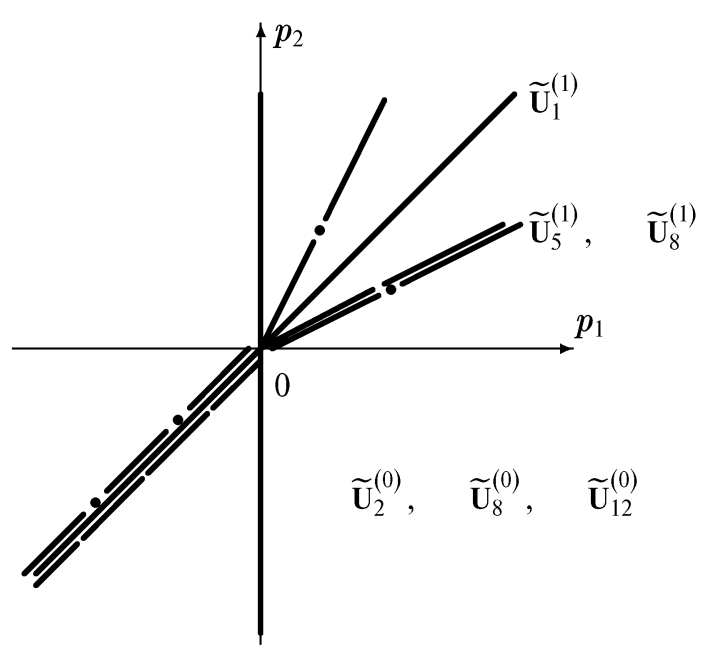

Figure 6 . The superimposed normal cones of the projections of the supports of the equations of the system (1.7)-(1.9).

for $P=(2,1,2,0,0)$; in the sixth column $(\mathrm{T})$ the sign "+" marks the maximal values of $\left\langle\widetilde{P}, \widetilde{Q}_{k}\right\rangle$ for given $i$ (the corresponding terms of the sum $f_{i}$ are included in the truncation $\left.\widehat{f}_{i 2}^{\left(d_{i}\right)}\right)$.

In the notation of Chapter I, $\S 1$, we have $l=2$ and the resulting vector is $P=\left(P^{\prime}, P^{\prime \prime}\right)$, that is, $P^{\prime}=(2,1)$. Furthermore, the vector $B_{1}^{\prime}=(-1,2)$ forms a basis in the space of vectors $Q^{\prime}=\left(q_{1}, q_{2}\right)$ satisfying the condition $\left\langle P^{\prime}, Q^{\prime}\right\rangle=0$. Then, according to Theorem 3 , Chapter I, we have $T_{3}^{\prime}=(1,0)$ and $T_{4}^{\prime}=T_{5}^{\prime}=0$, and according to (1.11) the self-similar coordinates $\xi, G, \mathrm{P}, H$ have the form

$$
\xi=\frac{r^{2}}{x}, \quad x_{3} \stackrel{\text { def }}{=} \psi=x G(\xi), \quad x_{4} \stackrel{\text { def }}{=} \rho=\mathrm{P}(\xi), \quad x_{5} \stackrel{\text { def }}{=} h=H(\xi),
$$

which corresponds to (1.19).

\section{A system of ODE}

From (1.16) and (1.19) it is clear that $\mathrm{P}(\xi) H(\xi)=$ const $=C_{0} \stackrel{\text { def }}{=} \rho_{0} h_{0} \neq 0$. Therefore,

$$
\mathrm{P}(\xi)=\frac{C_{0}}{H(\xi)} .
$$

After making the change of variables (1.19), (2.1) in equations (1.17) and (1.18) we obtain the system of ODE

$$
\begin{aligned}
& F_{2} \stackrel{\text { def }}{=} G\left(G^{\prime} H\right)^{\prime}+2 C^{n}\left[H^{n}\left(G^{\prime} H\right)^{\prime} \xi\right]^{\prime}=0, \\
& F_{3} \stackrel{\text { def }}{=} 2 G H^{\prime}+16 C^{n} C_{0}^{-2} \xi H^{n}\left(\left(G^{\prime} H\right)^{\prime}\right)^{2}+4 C^{n} \sigma^{-1}\left(\xi H^{n} H^{\prime}\right)^{\prime}=0,
\end{aligned}
$$

where $\stackrel{\text { def }}{=} d / d \xi$, with the boundary conditions

$$
\begin{gathered}
G=\psi_{0} \xi, \quad H=h_{0} \neq 0 \quad \text { as } \xi \rightarrow+\infty, \\
G=\frac{d G}{d \xi}=0 \quad \text { for } \xi=0 .
\end{gathered}
$$

We now observe that the equation

$$
\left(G^{\prime} H\right)^{\prime}=0
$$


or

$$
G^{\prime} H=c_{1}=\text { const } \neq 0,
$$

where $c_{1}$ is an arbitrary constant, distinguishes an invariant manifold of the complete system (2.2). On this manifold, the first equation of the system is satisfied identically, while the second, after factoring out 2, takes the form

$$
G H^{\prime}+2 C^{n} \sigma^{-1}\left(\xi H^{n} H^{\prime}\right)^{\prime}=0
$$

If $c_{1}=0$, then either $G^{\prime}=0$ or $H=0$. In either case the boundary condition (2.3) is not satisfied. Thus, we have obtained the system of two equations (2.6) and (2.7). We are interested in its solutions with the boundary conditions (2.3), (2.4). To normalize the constants we perform the linear change of coordinates

$$
\xi=\frac{C^{n} h_{0}^{n}}{\sigma \psi_{0}} \widetilde{\xi}, \quad G=\frac{C^{n} h_{0}^{n}}{\sigma} \widetilde{G}, \quad H=h_{0} \widetilde{H} .
$$

Then, omitting the tildes over the variables, we obtain the system of equations

$$
\begin{gathered}
G^{\prime} H=\widetilde{c}_{1}=\text { const, } \\
G H^{\prime}+2\left(\xi H^{n} H^{\prime}\right)^{\prime}=0
\end{gathered}
$$

with the boundary conditions (2.4) and

$$
G=\xi, \quad H=1 \quad \text { as } \quad \xi \rightarrow+\infty .
$$

It follows from the boundary condition (2.11) that $\widetilde{c}_{1}=1$ in equation (2.9) and it has the form

$$
G^{\prime} H=1 .
$$

Thus, we have obtained the system (2.12), (2.10) with boundary conditions (2.11), (2.4). We add equations (2.12) and (2.10) and integrate the sum to obtain

$$
G H+2 \xi H^{n} H^{\prime}=\xi+2 c_{2},
$$

where $c_{2}$ is an arbitrary constant. We now reduce the system of equations $(2.12),(2.13)$ to a single equation for $H$ by eliminating $G$. From (2.13) we obtain

$$
G=-2 \xi H^{n-1} H^{\prime}+\left(\xi+2 c_{2}\right) H^{-1} .
$$

Hence,

$$
G^{\prime}=-\frac{2\left(\xi H^{n} H^{\prime}\right)^{\prime}}{H}+\frac{2\left(\xi H^{n} H^{\prime}\right) H^{\prime}}{H^{2}}+\frac{1}{H}-\frac{\left(\xi+2 c_{2}\right) H^{\prime}}{H^{2}} .
$$

According to $(2.12)$ we have $G^{\prime}=1 / H$. Therefore in the last equation the terms $G^{\prime}$ and $1 / H$ cancel out. Multiplying equation $(2.14)$ by $-H^{2}$ we obtain the equation

$$
\Delta(\xi, H) \stackrel{\text { def }}{=} 2\left(\xi H^{n} H^{\prime}\right)^{\prime} H-2 \xi H^{n} H^{\prime 2}+\left(\xi+2 c_{2}\right) H^{\prime}=0
$$

with boundary conditions

$$
\begin{aligned}
H \rightarrow+\infty & \text { as } \xi \rightarrow+0 \\
H=1 & \text { as } \xi \rightarrow+\infty
\end{aligned}
$$

We call (2.15) the basic equation.

In addition, according to $\S 1$, because of the physical significance of enthalpy, we have $h>0$; consequently, we are only interested in those solutions $H(\xi)$ of equation (2.15) for which, according to (1.19) and (1.10),

$$
H(\xi)>0 \quad \text { and } \quad H^{n}>0 \quad \text { for } \xi \in(0, \infty) .
$$

Thus, we have obtained the following. 
Lemma 3. In the self-similar coordinates (1.19), after the change of variables (2.1), the problem (1.13), (1.14), (1.16)-(1.18) reduces to the problem (2.2)-(2.4). After the normalization (2.8), particular solutions of this problem are given by solutions of the problem (2.10)-(2.12). Eliminating $G$ according to (2.12), the latter problem reduces to equation (2.15) with the boundary conditions (2.16), (2.17) and with the property (2.18).

Remark 1. Equation (2.15) always has constant solutions

$$
H=\text { const } \stackrel{\text { def }}{=} H_{0} .
$$

Lemma 4. At any point $\xi=\xi^{0}=$ const, $H=H^{0}=$ const where $\xi^{0} \in(0, \infty), H^{0} \neq 0$, a non-constant solution $H(\xi)$ of equation (2.15) is monotonic.

Proof. We rewrite equation (2.15) in the form

$$
H^{\prime \prime}=\frac{2(1-n) H^{\prime 2}}{H}-\frac{H^{\prime}}{\xi}-\frac{\left(\xi+2 c_{2}\right) H^{\prime}}{2 \xi H^{n+1}} .
$$

According to Cauchy's theorem, a solution $H(\xi)$ of equation (2.15) is analytic at any point $\xi=\xi^{0}=$ const, $H=H^{0}=$ const, $H^{\prime}=H^{\prime 0}=$ const where $\xi^{0} \in(0, \infty), H^{0} \neq 0$. Consequently, it has an extremum at this point only if $H^{\prime 0}=0$. But then, according to $(2.20), H^{\prime \prime}=0$ at this point. Differentiating equation $(2.20)$ we see that all the higher derivatives $H^{(k)}$ also vanish at this point. An analytic function $H(\xi)$ all of whose derivatives vanish at some point is a constant.

\section{Solutions of the basic EQUation NeAR Zero}

Theorem 1. For equation (2.15) where $n \in[0,1]$ is fixed and $c_{2} \neq 0$, all the solutions satisfying the condition (2.16) form two two-parameter families and one one-parameter family. The two-parameter families are: the family $\mathcal{G}_{1}^{(0)}$, which exists for $n=0$ and has the expansions

$$
\begin{aligned}
& H=c_{4} \xi^{\lambda}+\sum_{s} a_{s} \xi^{s}, \quad \text { where } \lambda<0, \\
& s \in \mathbf{K}=\{s=\lambda-l \lambda+m(1-\lambda) ; \text { for integers } l, m \geq 0, l+m>0\},
\end{aligned}
$$

and the family $\mathcal{F}_{1}^{(1)} \mathcal{G}_{4}^{(0)}$ with the non-power asymptotics

$$
\begin{gathered}
H \sim \widetilde{c}|\ln \xi|^{1 / n}+\sum b_{s}|\ln \xi|^{s}, \quad s \in \mathbf{K}\left(k_{2}\right) \quad \text { for } n \in(0,1), \\
\mathbf{K}\left(k_{2}\right)=\{s=1 / n-l / n-m ; \text { for integers } l, m \geq 0, \quad l+m>0\}, \\
H \sim \widetilde{c} \ln \xi+\sum_{l=0}^{\infty} \beta_{l}(\ln |\ln \xi|)(\ln \xi)^{-l} \quad \text { for } n=1 .
\end{gathered}
$$

The one-parameter family $\mathcal{F}_{1}^{(1)} \mathcal{G}_{4}^{(1)}$ has the non-power asymptotics

$$
H \sim\left[(n+1)\left(c_{2} \ln \xi+c_{3}\right)\right]^{1 /(n+1)} ;
$$

it exists for $n \in[0,1]$ and $c_{2}<0$, and is the boundary of the preceding two-parameter families.

For $c_{2}=0$ in (3.1) the set $\mathbf{K}$ is equal to $\{s=\lambda+m(1-\lambda)$, for integers $m>0\}$, and the asymptotics (3.2) and (3.3) have the form $H \sim\left(c_{4} \ln \xi+c_{5}\right)^{1 / n}$ for $n \in(0,1]$.

Here, $\widetilde{c}, c_{3}, c_{4}, c_{5}$, and $\lambda<0$ are arbitrary constants, while the constants $a_{s}$ and $b_{s}$ are uniquely determined, except for $b_{-1+1 / n}$, which is arbitrary; the $\beta_{l}$ are polynomials in $\ln |\ln \xi|$ with constant coefficients, and all of them are uniquely determined, except for the polynomial $\beta_{0}$, which has an arbitrary constant term. The expansions in (3.1) and (3.2) converge if $|\xi|$ is small. 
Remark 2. According to [4], the asymptotics (3.2)-(3.4) can be extended into expansions of solutions in powers of $\xi$ where the coefficients depend on $\ln \xi$; when the expansion (3.3) is extended, the coefficients also depend on $\ln |\ln \xi|$. However, we do not need this in this paper.

Proof. We divide the proof into two cases: $c_{2} \neq 0$ and $c_{2}=0$. But first we observe that since $\xi \rightarrow 0$ and $H \rightarrow \infty$, the cone for the problem is

$$
p_{1} \leq 0, \quad p_{2} \geq 0 .
$$

Case $c_{2} \neq 0$. In this case the support of equation (2.15) consists of the three points $Q_{1}=(-1,2+n), Q_{2}=(0,1)$, and $Q_{3}=(-1,1)$. The support $\mathbf{S}(\Delta)$, the polygon $\Gamma(\Delta)$, and its faces $\Gamma_{j}^{(d)}$ for equation (2.15) are shown in Figure 7(a), and their normal cones $\mathbf{U}_{j}^{(d)}$ in Figure $7(\mathrm{~b})$, where the shaded area is the cone for the problem (3.5). According to Figure $\mathbf{7}(\mathrm{b})$, it intersects only the normal cones $\mathbf{U}_{1}^{(0)}$ and $\mathbf{U}_{1}^{(1)}$. We consider the corresponding faces and truncated equations, bearing in mind that $\omega=-1$ according to Chapter I, $\S 2.2$.

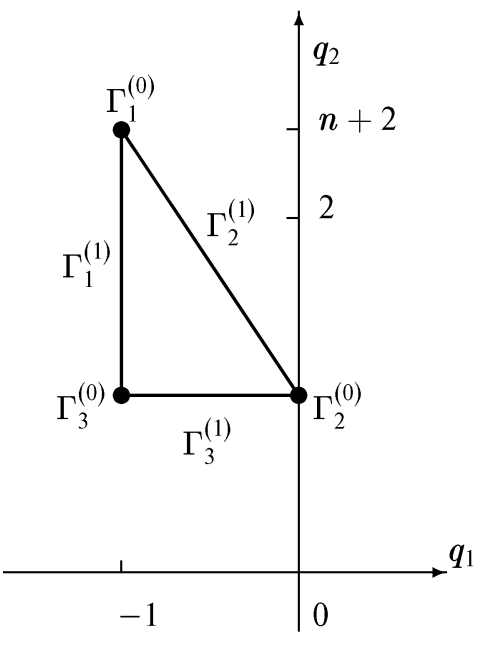

a)

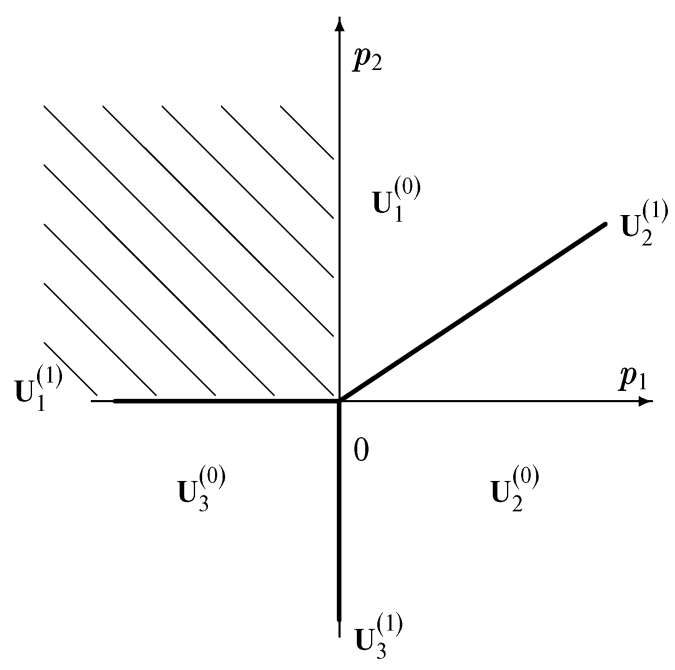

b)

Figure 7. (a) The support $\mathbf{S}(\Delta)$, the polygon $\Gamma(\Delta)$, and its faces $\Gamma_{j}^{(d)}$ for equation (2.15) for $c_{2} \neq 0$; (b) their normal cones. The shaded area is the cone for the problem (3.5).

The truncated equation

$$
\widehat{\Delta}_{1}^{(0)} \stackrel{\text { def }}{=} 2\left(\xi H^{n} H^{\prime}\right)^{\prime} H-2 \xi H^{n} H^{\prime 2}=2 H^{n}\left[(n-1) \xi H^{\prime 2}+\xi H H^{\prime \prime}+H H^{\prime}\right]=0
$$

corresponds to the vertex $\Gamma_{1}^{(0)}$. Equating the square brackets in equation (3.6) to zero and then dividing them by $\xi H H^{\prime}$ we obtain the equation

$$
(n-1) \frac{H^{\prime}}{H}+\frac{H^{\prime \prime}}{H^{\prime}}+\frac{1}{\xi}=0 .
$$

Integrating it with respect to $\xi$ we obtain the equation

$$
(n-1) \ln H+\ln \left|H^{\prime}\right|+\ln \xi=c_{0},
$$


where $c_{0}$ is an arbitrary constant. This equation is equivalent to

$$
H^{n-1} H^{\prime}=\frac{c_{1}}{\xi},
$$

where $c_{1} \neq 0$ is an arbitrary constant. Integrating this last equation we obtain

$$
\begin{aligned}
\frac{1}{n} H^{n} & =c_{1} \ln \xi+c_{3} & & \text { for } n \neq 0, \\
\ln H & =c_{1} \ln \xi+c_{3} & & \text { for } n=0,
\end{aligned}
$$

where $c_{3}$ is an arbitrary constant. These expressions are equivalent to the formulae

$$
\begin{gathered}
H=\left(c_{4} \ln \xi+c_{5}\right)^{1 / n} \quad \text { for } n \neq 0, \\
H=c_{4} \xi^{\lambda} \quad \text { for } n=0,
\end{gathered}
$$

where $c_{4}, c_{5}$, and $\lambda$ are arbitrary constants.

For the solutions (3.7), as $\xi \rightarrow 0$, the order is $r=0$, and according to Chapter I, $\S 2.2$ the vector $P=\omega(1, r)=-(1,0)$ corresponds to them, but does not belong to the normal cone $\mathbf{U}_{1}^{(0)}$. Consequently, there is no suitable solution (3.7) corresponding to the vertex $\Gamma_{1}^{(0)}$ for $n \neq 0$.

For the solutions (3.8), as $\xi \rightarrow 0$, the order is $r=\lambda$. If $\lambda<0$, which we assume in what follows, the vector $P=\omega(1, r)=(-1,-\lambda)$ belongs to $\mathbf{U}_{1}^{(0)}$. Consequently, for $n=0$ the expression (3.8) with $\lambda<0$ gives power asymptotics of solutions of equation (2.15). By Chapter I, $\S 2.3$ we find power expansions of these solutions of the form

$$
H=c_{4} \xi^{\lambda}+\sum_{s} b_{s} \xi^{s}, \quad s>\lambda, \quad s \in \mathbf{K} .
$$

The first variation of equation (3.6) for $n=0$ is

$$
\frac{\delta \widehat{\Delta}_{1}^{(0)}}{\delta H}=-4 \xi H^{\prime} \frac{d}{d \xi}+2 \xi H^{\prime \prime}+2 \xi H \frac{d^{2}}{d \xi^{2}}+2 H^{\prime}+2 H \frac{d}{d \xi} .
$$

By Theorem 5, Chapter I, on the curve (3.8) it gives the operator

$$
\mathcal{L}(\xi)=2 c_{4} \xi^{\lambda-1}\left[-2 \lambda \xi \frac{d}{d \xi}+\lambda(\lambda-1)+\xi^{2} \frac{d^{2}}{d \xi^{2}}+\lambda+\xi \frac{d}{d \xi}\right] \not \equiv 0 .
$$

Then the characteristic polynomial of the differential sum $\mathcal{L}(\xi) \xi^{k}$ is

$$
\nu(k)=2 c_{4}[-2 \lambda k+\lambda(\lambda-1)+k(k-1)+\lambda+k]=2 c_{4}(k-\lambda)^{2} .
$$

It has one double root $k_{1}=\lambda=r$, which is not a critical number, since it does not satisfy the inequality $k_{1}>r$.

To calculate the set $\mathbf{K}$ we use Lemma 1 in Chapter I, $\S 2$. Here,

$$
M_{1}=(0,-1), \quad M_{2}=(1,-1) .
$$

Therefore,

$$
r_{1} \stackrel{\text { def }}{=}\left\langle M_{1},(1, r)\right\rangle=-\lambda, \quad r_{2} \stackrel{\text { def }}{=}\left\langle M_{2},(1, r)\right\rangle=1-\lambda .
$$

Consequently,

$$
\mathbf{K}=\{s=\lambda-l \lambda+m(1-\lambda) ; \text { for integers } l, m \geq 0, l+m>0\} .
$$

In the expansions $(3.9),(3.11)$, all the coefficients $b_{s}$ are constant and uniquely determined; according to Theorem 10, Chapter I, the expansions converge for sufficiently small $|\xi| \neq 0$. We denote the family they form by $\mathcal{G}_{1}^{(0)}$. Thus, we have obtained the expansion (3.1). 
To the edge $\Gamma_{1}^{(1)}$ there corresponds the truncated equation

$$
\widehat{\Delta}_{1}^{(1)} \stackrel{\text { def }}{=} 2\left(\xi H^{n} H^{\prime}\right)^{\prime} H-2 \xi H^{n} H^{\prime 2}+2 c_{2} H^{\prime}=0 .
$$

This equation has the invariant manifold

$$
\xi H^{n} H^{\prime}=c_{2} .
$$

On it, $H^{n} H^{\prime}=c_{2} / \xi$. By integrating this equation with respect to $\xi$ we obtain

$$
\frac{H^{n+1}}{n+1}=c_{2} \ln \xi+c_{3}
$$

where $c_{3}$ is an arbitrary constant. Thus,

$$
H=\left[(n+1)\left(c_{2} \ln \xi+c_{3}\right)\right]^{1 /(n+1)} .
$$

For the solutions (3.14) to be real it is necessary that $c_{2}<0$, since $\ln \xi<0$ as $\xi \rightarrow 0$. Thus, we have obtained the expansion (3.4).

We now find the solutions of equation (3.12) satisfying condition (2.16). The edge $\Gamma_{1}^{(1)}$ is vertical, as is clear from Figure 7(a); consequently, the power solutions are $H=$ const, that is, (2.19), but they do not satisfy condition (2.16). To find non-power solutions, according to Chapter I, $\S 2.7$ we make the logarithmic transformation

$$
t=\ln \xi
$$

and denote the derivative with respect to $t$ by a dot: $\stackrel{\text { def }}{=} d / d t$. Then $H^{\prime}=\dot{H} / \xi$, and equation (3.12) takes the form

$$
\Omega(t, H) \stackrel{\text { def }}{=} 2\left[\frac{d H^{n}}{d t} \dot{H}\right] H-2 H^{n} \dot{H}^{2}+2 c_{2} \dot{H}=0 .
$$

Its support $\mathbf{S}(\Omega)$ consists of the two points $Q_{4}=(-2,2+n)$ and $Q_{5}=(-1,1)$. The polygon $\Gamma(\Omega)$ is the segment that connects them. The vector $N_{4}=(1,1 /(n+1))$ is normal to it. Its faces $\Gamma_{j}^{(d)}$ are shown in Figure $8(a)$, and their normal cones in Figure 8(b).

We set $\Gamma_{j}^{(0)}=Q_{j}$. Here, $t \rightarrow-\infty$, that is, $\omega=1$, and the cone for the problem is $p_{1} \geq 0, p_{2} \geq 0$ (shaded in Figure $8(\mathrm{~b})$ ). It intersects all three normal cones $\mathbf{U}_{j}^{(d)}$. We consider the solutions corresponding to the faces $\Gamma_{j}^{(d)}$.

Corresponding to the vertex $\Gamma_{4}^{(0)}$ we have the truncated equation

$$
\widehat{\Omega}_{4}^{(0)} \stackrel{\text { def }}{=} 2\left[\frac{d H^{n}}{d t} \dot{H}\right] H-2 H^{n} \dot{H}^{2}=2\left[(n-1) H^{n} \dot{H}^{2}+H^{n+1} \ddot{H}\right]=0 .
$$

In fact, this is equation (3.6) with the independent variable $t=\ln \xi$. Its characteristic polynomial is

$$
2 r(n r+r-1)-2 r^{2}=2 r(n r-1) .
$$

It has the two roots $r=0$ and $r=1 / n$. The corresponding vectors $\omega(1, r)$ are $P_{1}=(1,0)$ and $P_{2}=(1,1 / n)$. The vector $P_{1}$ does not belong to the normal cone $\mathbf{U}_{4}^{(0)}$, while the vector $P_{2}$ belongs to $\mathbf{U}_{4}^{(0)}$ since $1 / n>1 /(n+1)$. Consequently, for $n \neq 0$ the truncated equation (3.17) has the power solutions

$$
H=\widetilde{c} t^{1 / n},
$$

where $\widetilde{c} \neq 0$ is an arbitrary constant (possibly complex). We now find their critical numbers. According to Chapter I, $\S 2.3$, the first variation is

$$
\frac{\delta \widehat{\Omega}_{4}^{(0)}}{\delta H}=2\left[(n-1) n H^{n-1} \dot{H}^{2}+2(n-1) H^{n} \dot{H} \frac{d}{d t}+(n+1) H^{n} \ddot{H}+H^{n+1} \frac{d^{2}}{d t^{2}}\right] .
$$




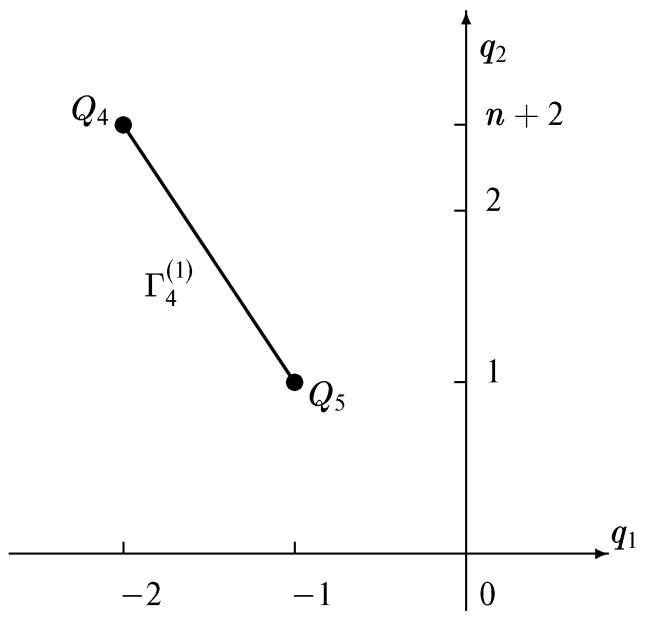

a)

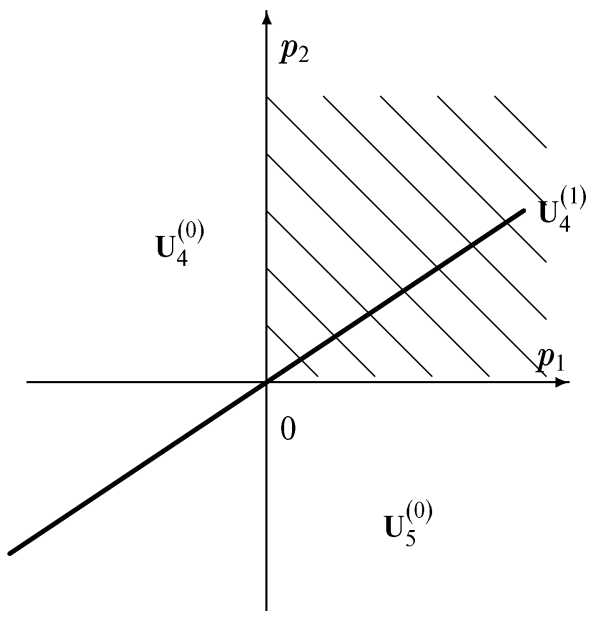

b)

Figure 8. (a) The faces $\Gamma_{j}^{(d)}$ of the polygon of equation (3.16) for $c_{2} \neq 0$; (b) their normal cones.

On the curve (3.18) it gives the operator

$$
\mathcal{L}(t)=2 \widetilde{c}^{n+1} t^{(1-n) / n}\left[\frac{n-1}{n}+\frac{n+1}{n}\left(\frac{1}{n}-1\right)+2 \frac{n-1}{n} t \frac{d}{d t}+t^{2} \frac{d^{2}}{d t^{2}}\right] .
$$

The characteristic polynomial of the differential sum $\mathcal{L}(t) t^{k}$ is

$$
\nu(k)=2 \widetilde{c}^{n+1}\left[k(k-1)+\frac{2(n-1)}{n} k+\frac{1-n}{n^{2}}\right] .
$$

It has the two roots $k_{1}=1 / n$ and $k_{2}=(1-n) / n=k_{1}-1$. Here the cone for the problem is $k<r=1 / n$. Since $k_{1}=r$, this root is not a critical number. But $k_{2}=r-1<r$ and therefore $k_{2}$ is a critical number. According to Lemmas 1 and 2 in Chapter I, here the corresponding sets are

$$
\begin{gathered}
\mathbf{K}=\{s=-l / n, \text { for integers } l \geq 0\} \\
\mathbf{K}\left(k_{2}\right)=\{s=1 / n-l / n-m, \text { for integers } l, m \geq 0, l+m>0\} .
\end{gathered}
$$

If $n \in(0,1)$, then $k_{2}=1 / n-1 \notin \mathbf{K}$ and equation (3.16) has the family $\mathcal{G}_{4}^{(0)}$ of power expansions of solutions

$$
H=\widetilde{c}|t|^{1 / n}+\sum b_{s}|t|^{s}, \quad s \in \mathbf{K}\left(k_{2}\right),
$$

where the $b_{s}$ are constant coefficients, $\widetilde{c}$ and $b_{k_{2}}$ are arbitrary, while the other coefficients $b_{s}$ are uniquely determined. According to Chapter I, Theorem 10 the expansion (3.20) converges for sufficiently large $|t|$. If we set $b_{k_{2}}=0$, then the expansion (3.20) has the form

$$
H=\widetilde{c}|t|^{1 / n}+\sum_{l=0}^{\infty} b_{l}|t|^{-l / n}
$$


where $\widetilde{c}$ is an arbitrary constant, while the constants $b_{l}$ are uniquely determined. For example,

$$
b_{0}=\frac{n c_{2}}{\widetilde{c}^{n}(n-1)}, \quad b_{1}=\frac{n(n-1) b_{0}^{2}}{2(2-n) \widetilde{c}}=\frac{n^{3} c_{2}^{2}}{2(2-n)(n-1) \widetilde{c}^{2 n+1}} .
$$

According to (3.14), the expansion (3.20) gives the two-parameter family $\mathcal{F}_{1}^{(1)} \mathcal{G}_{4}^{(0)}$ of non-power asymptotics of solutions of equation (2.15)

$$
H \sim \widetilde{c}|\ln \xi|^{1 / n}+\sum b_{s}|\ln \xi|^{s}, \quad s \in \mathbf{K}\left(k_{2}\right) .
$$

Thus, we have obtained the expansion (3.2).

If $n=1$, then $k_{2}=0 \in \mathbf{K}$ and, according to Chapter I, $\S 2.5$, equation (3.16) has a family of power-logarithmic expansions of the form (3.21), where the coefficients $b_{l}$ are polynomials in $\ln |t|$, the coefficient $b_{0}$ contains an arbitrary constant, while the other coefficients $b_{l}$ are uniquely determined. This family is also denoted by $\mathcal{G}_{4}^{(0)}$. The twoparameter family $\mathcal{F}_{1}^{(1)} \mathcal{G}_{4}^{(0)}$ of non-power asymptotic solutions of equation (2.15) also corresponds to it. Thus, we have obtained the expansion (3.3).

Furthermore, we can integrate equation (3.17) explicitly, because equation (3.17) is obtained from (3.6) after the change of variables (3.15), and the solutions of equation (3.6) are (3.7) and (3.8).

The truncated equation $\widehat{\Omega}_{5}^{(0)} \stackrel{\text { def }}{=} 2 c_{2} \dot{H}=0$ corresponds to the vertex $\Gamma_{5}^{(0)}$. Its solutions are all given by $H=$ const, that is, $r=0$. The vector $P=\omega(1, r)=(1,0)$ belongs to $\mathbf{U}_{5}^{(0)}$ (see Figure $8(\mathrm{~b})$ ). We cannot use the corresponding power truncated solutions (2.19), because they do not satisfy the boundary condition (2.16). Therefore the solutions corresponding to the vertex $\Gamma_{5}^{(0)}$ are not suitable.

The whole equation (3.16) corresponds to the edge $\Gamma_{4}^{(1)}$. Since $N_{4}=(1,1 /(n+1))$ is a normal vector to the edge $\Gamma_{4}^{(1)}$, we seek solutions of equation (3.16) in the form

$$
H=\widetilde{c}_{0} t^{1 /(n+1)} .
$$

For $\widetilde{c}_{0}$ we obtain the defining equation

$$
\widetilde{c}_{0}^{n+2} \frac{1}{n+1}\left(\frac{n}{n+1}+\frac{1}{n+1}-1\right)-\widetilde{c}_{0}^{n+2} \frac{1}{(n+1)^{2}}+c_{2} \widetilde{c}_{0} \frac{1}{n+1}=0 .
$$

From it we obtain that $\widetilde{c}_{0}^{n+1}=(n+1) c_{2}$, that is,

$$
\widetilde{c}_{0}=\left[(n+1) c_{2}\right]^{1 /(n+1)} \text {. }
$$

Consequently,

$$
H=\left[(n+1) c_{2} \ln \xi\right]^{1 /(n+1)} .
$$

We have $\ln \xi<0$ as $\xi \rightarrow 0$; therefore there only exist solutions with the property (2.18) if $c_{2}<0$; they do not exist for $c_{2} \geq 0$.

We now find the critical numbers of the truncated solution (3.22). According to (3.16) and (3.19), the first variation is

$$
\frac{\delta \Omega}{\delta H}=2\left[(n-1) n H^{n-1} \dot{H}^{2}+2(n-1) H^{n} \dot{H} \frac{d}{d t}+(n+1) H^{n} \ddot{H}+H^{n+1} \frac{d^{2}}{d t^{2}}+c_{2} \frac{d}{d t}\right] .
$$

On the curve (3.22) it gives the operator

$$
\mathcal{L}(t)=2\left\{\widetilde{c}_{0}^{n+1}\left[\frac{(n-1) n}{(n+1)^{2}} t^{-1}+\frac{2(n-1)}{n+1} \frac{d}{d t}+\frac{(n+1)}{(n+1)}\left(\frac{1}{n+1}-1\right) t^{-1}+t \frac{d^{2}}{d t^{2}}\right]+c_{2} \frac{d}{d t}\right\} .
$$


According to (3.24), the characteristic polynomial of the differential sum $\mathcal{L}(t) t^{k}$ is

$$
\begin{aligned}
\nu(k) & =2 c_{2}\left\{(n+1)\left[\frac{(n-1) n}{(n+1)^{2}}-\frac{(n+1) n}{(n+1)^{2}}+\frac{2(n-1)}{n+1} k+k(k-1)\right]+k\right\} \\
& =2 c_{2}(n+1)\left\{-\frac{2 n}{(n+1)^{2}}+\frac{n-2}{n+1} k+k^{2}\right\} .
\end{aligned}
$$

Its roots are $k_{1}=2 /(n+1)$ and $k_{2}=-n /(n+1)$. Here the cone for the problem is $s<r=1 /(n+1)$. The root $k_{1}>r$ is not a critical number, but the root $k_{2}<r$ is.

The expression (3.22), where (3.24) holds, is an exact solution of equation (3.16). According to Lemma 1, Chapter I, the set $\mathbf{K}$ is empty; consequently, $k_{2} \notin \mathbf{K}$. By Lemma 2, Chapter I,

$$
\mathbf{K}\left(k_{2}\right)=\left\{r+l\left(k_{2}-r\right)\right\}=\left\{r+l\left(-\frac{n}{n+1}-\frac{1}{n+1}\right)\right\}=\{r-l\},
$$

where $l>0$ is an integer. Consequently, there is a one-parameter family $\mathcal{G}_{4}^{(1)}$ of power expansions

$$
H=\widetilde{c}_{0} t^{1 /(n+1)}\left(1+\sum_{l=1}^{\infty} b_{l} t^{-l}\right),
$$

where the coefficients $b_{l}$ are constant. The coefficient $b_{1}$, which corresponds to the critical number $k_{2}$, is an arbitrary constant, while the other $b_{l}$ are uniquely determined. According to Theorem 10, Chapter I, the expansion converges for sufficiently large $|t|$.

On the other hand, on the invariant manifold (3.13) equation (3.12) has a one-parameter family of solutions (3.14) (with respect to $c_{3}$ ). Comparing it with the family $(3.24),(3.25)$ shows that they coincide. Thus, we have obtained the family $\mathcal{F}_{1}^{(1)} \mathcal{G}_{4}^{(1)}$ with the asymptotics (3.4).

According to Remark 4, Chapter I, since the defining equation (3.23) has no repeated, zero, or infinite roots, the solutions of equation (3.16) have no non-power asymptotics.

In addition, we can reduce the order of equation (3.16). For that we make the change of variables $\dot{H}=p, \ddot{H}=p^{\prime} p$ in equation (3.16), where $p^{\prime}=d p / d H$. Then its solutions are

$$
p=c_{2} H^{-n}+c_{0} H^{1-n},
$$

where $c_{0}$ is an arbitrary constant. Since $p=\dot{H}$, we obtain

$$
\dot{H}=c_{2} H^{-n}+c_{0} H^{1-n} \text {. }
$$

The resulting equation (3.26) is of lower order than the original equation (3.16). For some values of $n$ and $c_{0}$ this equation can be integrated in finite form.

For $n=0$ this equation is $\dot{H}=c_{2}+c_{0} H$, that is,

$$
H=\frac{c_{3} \xi^{c_{0}}-c_{2}}{c_{0}}
$$

where $c_{3}$ is an arbitrary constant.

For $n=1$ equation (3.26) takes the form $\dot{H}=c_{2} H^{-1}+c_{0}$, that is,

$$
c_{0} H-c_{2} \ln \left|c_{0} H+c_{2}\right|=c_{0}^{2} t+c_{4},
$$

where $c_{4}$ is an arbitrary constant.

For $c_{0}=0$ equation (3.26) takes the form $\dot{H}=c_{2} H^{-n}$, that is,

$$
H=\left[c_{2}(n+1) \ln \xi+c_{4}\right]^{1 /(n+1)},
$$

where $c_{4}$ is an arbitrary constant. This is formula (3.4). 
The case $\boldsymbol{c}_{\mathbf{2}}=\mathbf{0}$. In this case the support $\mathbf{S}(\Delta)$ of equation (2.15) consists of the two points $Q_{1}=(-1, n+2)$ and $Q_{2}=(0,1)$; the polygon $\Gamma(\Delta)$ is a segment. Figure 9 (a) shows the support and polygon of equation (2.15) for $c_{2}=0$, and Figure 9(b) the normal cones of its faces, with the cone for the problem (3.5) shaded. It intersects only the normal cone $\mathbf{U}_{1}^{(0)}$. We consider the corresponding vertex and the truncated equation.

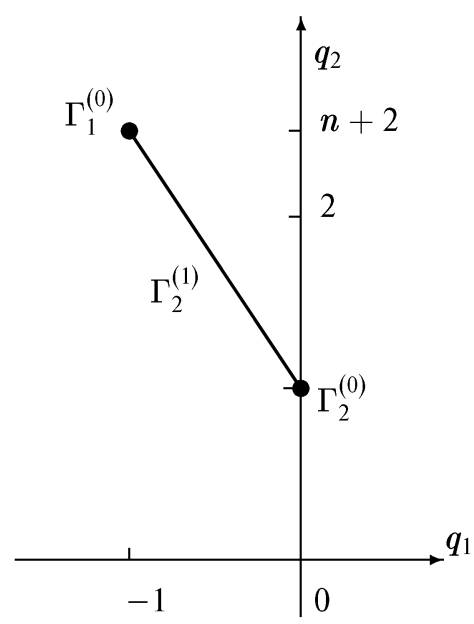

a)

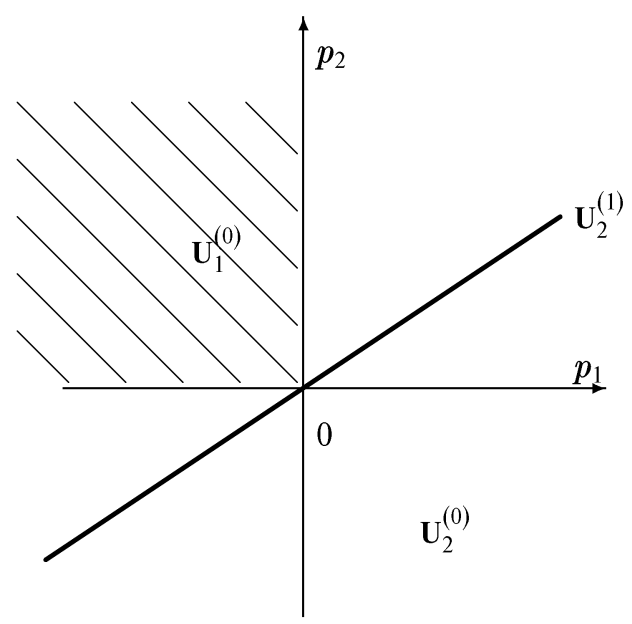

b)

Figure 9. (a) The support and polygon of equation (2.15) for $c_{2}=0$;

(b) the normal cones of its faces.

The truncated equation (3.6) corresponds to the vertex $\Gamma_{1}^{(0)}=Q_{1}$. We found all its solutions above when we looked at the case $c_{2} \neq 0$; they are described by formulae (3.7), (3.8). For the solutions $(3.7)$, the vector $P_{1}=\omega(1,0)=(-1,0)$ belongs to the normal cone $\mathbf{U}_{1}^{(0)}$. Therefore (3.7) gives the non-power asymptotics of solutions of equation (2.15) as $\xi \rightarrow 0$. Furthermore, for $c_{4}=0$, (3.7) also gives the power asymptotics of solutions near $\xi=0, H=H_{0}=$ const.

For the solutions $(3.8)$ the vector $P_{2}=\omega(1, \lambda)=(-1,-\lambda)$ belongs to $\mathbf{U}_{1}^{(0)}$ if $\lambda<1$. The power expansions of the form (3.9), which we have found, are valid if formula (3.10) holds, as it was used to calculate the set $\mathbf{K}$. In this case the vectors are $M_{1}=M_{2}=$ $(1,-1)$. Therefore, $r_{1}=r_{2}=1-\lambda$. Consequently,

$$
\mathbf{K}=\{s=\lambda+m(1-\lambda), \text { for integers } m>0\} .
$$

For $\lambda \geq 0$ the expansion (3.1) with index set (3.27) does not satisfy the boundary condition (2.16), that is, there only remains the possibility that $\lambda<0$.

Thus, the solutions form a two-parameter family, which for $n=0$ is represented by the expansions (3.9), (3.27) with $c_{4} \neq 0, \lambda<0$, and for $n \neq 0$ it is represented by asymptotics of the form (3.7) with $c_{4} \neq 0$.

Theorem 1 is proved.

Lemma 5. For $c_{2} \geq 0$ equation (2.15) has the one-parameter family of solutions (2.19). For $c_{2}<0$ equation (2.15) has a two-parameter family of solutions, which tend to constants $H_{0} \neq 0$ as $\xi \rightarrow 0$; this family is denoted by $\mathcal{F}_{1}^{(1)} 1$. 
Proof. The truncated equation (3.12) also has the constant solutions (2.19), which for $H_{0} \neq 0$ can be regarded as power asymptotics. We now find the solutions of equation (2.15) near the solution (2.19) as $\xi \rightarrow 0$. According to Chapter I, $\S 2.2$, we have $r=0$ and $c_{r}$ is an arbitrary constant. According to (3.6), the first variation of equation $(3.12)$ is

$$
\begin{aligned}
\frac{\delta \widehat{\Delta}_{1}^{(1)}}{\delta H}= & 2 n(n-1) \xi H^{n-1} H^{\prime 2}+4(n-1) \xi H^{n} H^{\prime} \frac{d}{d \xi}+2(n+1) \xi H^{n} H^{\prime \prime} \\
& +2 \xi H^{n+1} \frac{d^{2}}{d \xi^{2}}+2(n+1) H^{n} H^{\prime}+2 H^{n+1} \frac{d}{d \xi}+2 c_{2} \frac{d}{d \xi} .
\end{aligned}
$$

By Theorem 5, Chapter I, on the solution (2.19) with $H_{0} \neq 0$ it gives the operator

$$
\mathcal{L}(\xi)=2 \xi H_{0}^{n+1} \frac{d^{2}}{d \xi^{2}}+2 H_{0}^{n+1} \frac{d}{d \xi}+2 c_{2} \frac{d}{d \xi} .
$$

The characteristic polynomial of the differential sum $\mathcal{L}(\xi) \xi^{k}$ is

$$
\nu(k)=2 H_{0}^{n+1} k\left(k+\frac{c_{2}}{H_{0}^{n+1}}\right) .
$$

It has the two roots $k_{1}=0$ and $k_{2}=-c_{2} / H_{0}^{n+1}$. The eigenvalue $k_{1}=r=0$ is not a critical number. Since the cone for the problem is $\mathcal{K}=\{s>r=0\}$, the root $k_{2}$ is a critical number only if $-c_{2} / H_{0}^{n+1}>0$. Since $H_{0}$ and $H_{0}^{n+1}$ are positive, this means that $c_{2}<0$.

If there are no critical numbers (that is, $c_{2} \geq 0$ ), then a unique solution of equation (2.15) of the form

$$
H=H_{0}+\sum_{k=1}^{\infty} c_{k} \xi^{k}
$$

comes from the point $\xi=0, H=H_{0} \neq 0$ where all the $c_{k}$ are uniquely determined. By Remark 1 before Lemma 4 , the solution $H=H_{0} \stackrel{\text { def }}{=}$ const comes out from this point. Consequently, all the $c_{k}$ in formula (3.28) are zeros.

If there is one critical number $k_{2}$ (that is, $c_{2}<0$ ), then we have two subcases: integer and non-integer $k_{2}$. If the critical number $k_{2}$ is not an integer, that is, $k_{2} \notin \mathbf{K}=\{k=l$, for integers $l>0\}$, then by Lemma 2, Chapter I, a one-parameter family of solutions of equation (2.15) with the expansion

$$
H=H_{0}+\sum_{s} c_{s} \xi^{s}, \quad s \in \mathbf{K}\left(k_{2}\right),
$$

comes out from the point $\xi=0, H=H_{0} \neq 0$, where

$$
\mathbf{K}\left(k_{2}\right)=\left\{l+m k_{2} \text {, for integers } l, m \geq 0, l+m>0\right\}
$$

and the constants $c_{s}$ are uniquely determined, except for $s=k_{2}$, when $c_{s}$ is arbitrary.

If the critical number $k_{2}$ is an integer, then $k_{2} \in \mathbf{K}$. Consequently, a one-parameter family of solutions of equation (2.15) comes out from the point $\xi=0, H=H_{0} \neq 0$, and has the expansion

$$
H=H_{0}+\sum_{s} \gamma_{s} \xi^{s}, \quad s \in \mathbf{K},
$$

where the coefficients $\gamma_{s}$ for $s<k_{2}$ are constants, which are uniquely determined; the $\gamma_{s}$ for $s \geq k_{2}$ are polynomials in $\ln \xi$; the coefficient $\gamma_{k_{2}}$ contains an arbitrary constant, while the other $\gamma_{s}$ are uniquely determined. 


\section{Solutions of THE BASIC EQUATION NEAR INFINITY}

Theorem 2. For fixed $n$ and $c_{2}$ and as $\xi \rightarrow \infty$, equation (2.15) has a one-parameter family $\mathcal{M}$ of solutions with $H \rightarrow 1$. Their asymptotics are given by the formulae

$$
H^{\prime} \sim c_{10} \xi^{s} e^{-\xi / 2}, \quad H-1 \sim c_{10} \int \xi^{s} e^{-\xi / 2} d \xi,
$$

where $s=-c_{2}-1$ and $c_{10}$ is an arbitrary constant. The integral in (4.1) satisfies the asymptotic expansion

$$
H-1 \sim-2 c_{10} e^{-\xi / 2}\left[\xi^{s}+\sum_{l=1}^{\infty} 2^{l} s(s-1) \ldots(s-l+1) \xi^{s-l}\right] .
$$

Proof. We observe that $H=1$ is a solution of equation (2.15). In equation (2.15) we set $H=1+y$ and consider the equation

$$
\text { (4.3) } \Sigma(\xi, y) \stackrel{\text { def }}{=} \Delta(\xi, 1+y) \stackrel{\text { def }}{=} 2\left[\xi(1+y)^{n} y^{\prime}\right]^{\prime}(1+y)-2 \xi(1+y)^{n} y^{\prime 2}+\left(\xi+2 c_{2}\right) y^{\prime}=0 \text {. }
$$

It has the trivial solution $y=0$. The polygon $\Gamma(\Sigma)$ and the normal cones of its faces are shown in Figure 10. We are interested in the solutions such that $y \rightarrow 0$ as $\xi \rightarrow \infty$. Consequently, the cone for the problem is $\mathcal{K}=\left\{p_{1} \geq 0, p_{2} \leq 0\right\}$ (shaded in Figure 10(b)). The polygon $\Gamma(\Sigma)$ has the horizontal edge $\Gamma_{1}^{(1)}$ and the vertex $\Gamma_{1}^{(0)}$ with $q_{2}=1$ (Figure 10(a)). But the vertical edge with $q_{1}=0$ is improper, that is, its own truncated equation and normal cone do not correspond to it. The cone for the problem intersects the normal cones $\mathbf{U}_{1}^{(0)}$ and $\mathbf{U}_{1}^{(1)}$. We consider the truncated equations corresponding to the vertex $\Gamma_{1}^{(0)}$ and edge $\Gamma_{1}^{(1)}$.

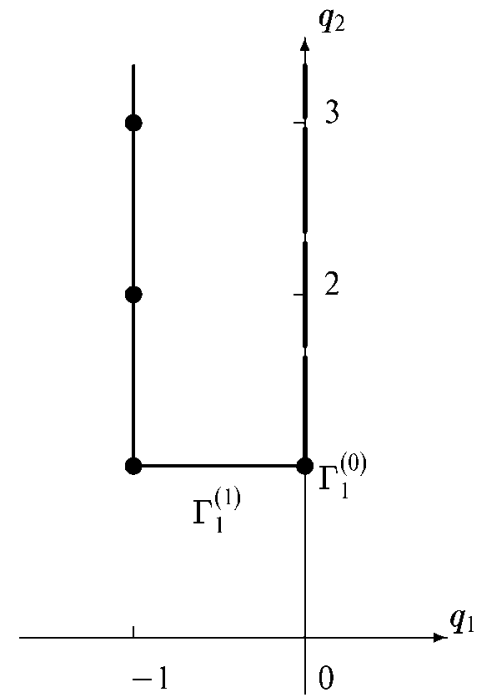

a)

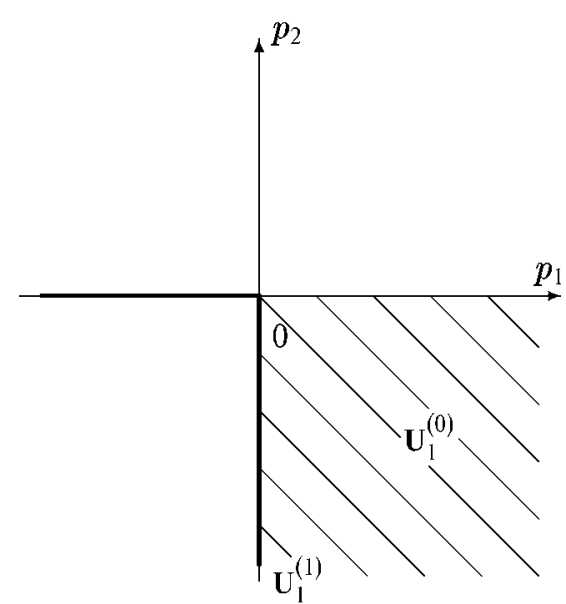

b)

Figure 10. (a) The support and polygon of equation (4.3), the normal cones of its faces; (b) the cone for the problem.

Corresponding to the vertex $\Gamma_{1}^{(0)}$ we have the truncated equation $\xi y^{\prime}=0$. All its solutions are $y=c_{0}=$ const. Since we are interested in the solutions such that $y \rightarrow 0$ as $\xi \rightarrow \infty$, we cannot use these solutions when $c_{0} \neq 0$. 
The truncated equation

$$
\widehat{\Sigma}_{1}^{(1)}(\xi, y) \stackrel{\text { def }}{=} 2\left[\xi y^{\prime}\right]^{\prime}+\left(\xi+2 c_{2}\right) y^{\prime}=0
$$

corresponds to the edge $\Gamma_{1}^{(1)}$, that is,

$$
\widehat{\Sigma}_{1}^{(1)}(\xi, y) \stackrel{\text { def }}{=} 2 \xi y^{\prime \prime}+\left(\xi+2\left(c_{2}+1\right)\right) y^{\prime}=0 .
$$

According to $\S 2.9$ of Chapter I, after the change of variables $\eta=d \ln y^{\prime} / d \xi$, equation (4.4) takes the form $2 \xi \eta+2\left(c_{2}+1\right)+\xi=0$. Its solution is $\eta=-1 / 2+\left(-c_{2}-1\right) / \xi$, that is, $y^{\prime}=c_{10} \xi^{s} e^{-\xi / 2}$, where $s=-c_{2}-1$ and $c_{10}$ is an arbitrary constant. This solution is equivalent to formulae (4.1). The second of them satisfies the asymptotic expansion (4.2). Indeed, integrating the integral in (4.1) by parts we obtain

$$
\int \xi^{s} e^{-\xi / 2} d \xi=-2 e^{-\xi / 2} \xi^{s}+2 s \int \xi^{s-1} e^{-\xi / 2} d \xi
$$

The resulting integral is again evaluated by parts; we obtain the first term of the sum in (4.2) and the integral

$$
\int \xi^{s} e^{-\xi / 2} d \xi=-2 e^{-\xi / 2}\left[\xi^{s}+2 s \xi^{s-1}\right]+4 s(s-1) \int \xi^{s-2} e^{-\xi / 2} d \xi .
$$

By continuing this process we obtain the asymptotic expansion (4.2).

For non-negative integer $s$, that is, for

$$
c_{2}=-1,-2, \ldots,
$$

the sum in the square brackets in (4.2) is finite, that is, this is a polynomial of degree $s$.

For fixed $n$, all the points in the $c_{2}, c_{10}$-plane except the straight line $c_{10}=0$ correspond to the family $\mathcal{M}$.

\section{Solutions of the basic equation near a point $\xi^{0}>0$}

Lemma 6. Solutions of equation (2.15) do not go to infinity and do not come from infinity for any finite $\xi^{0}>0$.

Proof. In equation (2.15) we make the substitution

$$
\xi=\xi^{0}+\widetilde{\xi},
$$

where $\xi^{0}=$ const $>0$. Then equation (2.15) takes the form

$$
\begin{aligned}
f(\widetilde{\xi}, H) \stackrel{\text { def }}{=} & \Delta\left(\xi^{0}+\widetilde{\xi}, H\right) \\
= & 2\left[\left(\xi^{0}+\widetilde{\xi}\right) H^{n} H^{\prime}\right]^{\prime} H-2\left(\xi^{0}+\widetilde{\xi}\right) H^{n} H^{\prime 2}+\left(\xi^{0}+\widetilde{\xi}+2 c_{2}\right) H^{\prime} \\
= & 2(n-1)\left(\xi^{0}+\widetilde{\xi}\right) H^{n} H^{\prime 2}+2\left(\xi^{0}+\widetilde{\xi}\right) H^{n+1} H^{\prime \prime}+2 H^{n+1} H^{\prime} \\
& +\left(\xi^{0}+\widetilde{\xi}+2 c_{2}\right) H^{\prime} \\
= & 0 .
\end{aligned}
$$

If $\xi^{0}+2 c_{2} \neq 0$, then its support consists of the points $Q_{1}, Q_{2}, Q_{3}$ and the point $Q_{6}=$ $(-2,2+n)$; the polygon $\Gamma(f)$ is a parallelogram (Figure 11(a)). We are interested in the solutions of equation (5.2) such that $H \rightarrow \infty$ as $\widetilde{\xi} \rightarrow 0$, that is, the cone for the problem is $p_{1} \leq 0, p_{2} \geq 0$. It intersects only the two normal cones $\mathbf{U}_{6}^{(0)}$ and $\mathbf{U}_{6}^{(1)}$ (Figure 11(b)). We consider the corresponding faces and truncated equations.

The truncated equation

$$
\widehat{f}_{6}^{(0)} \stackrel{\text { def }}{=} 2(n-1) \xi^{0} H^{n} H^{2}+2 \xi^{0} H^{n+1} H^{\prime \prime}=0
$$




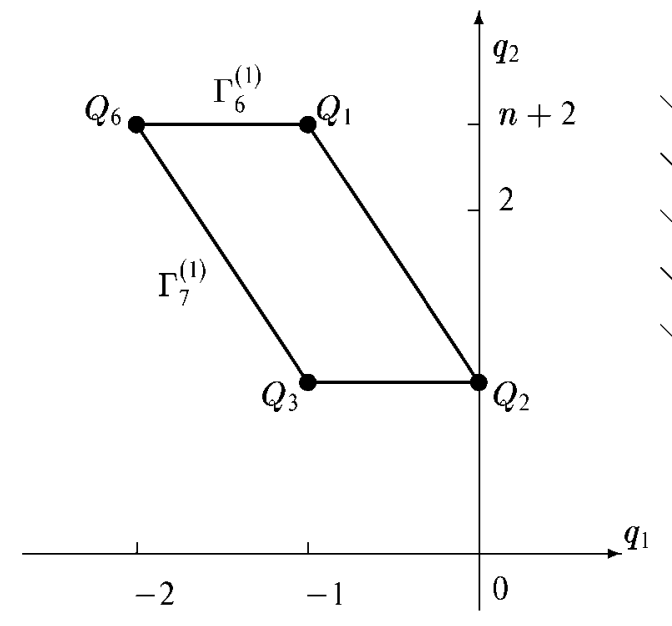

a)

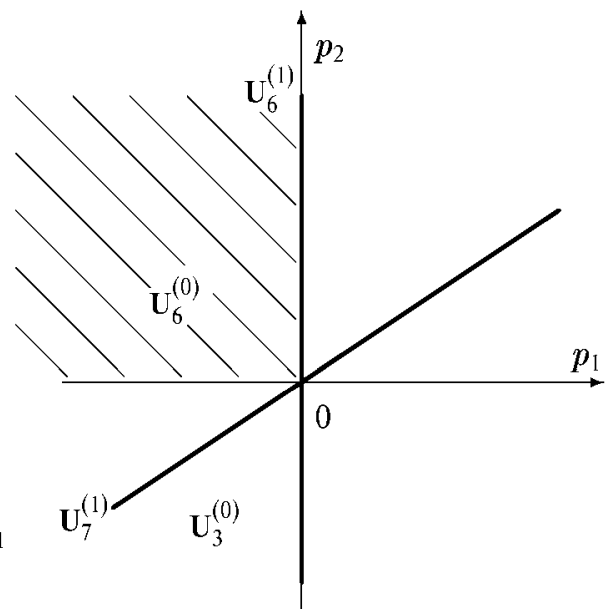

b)

FigurE 11. (a) The support and the polygon of equation (5.2), and the normal cones of its faces; (b) the cone for the problem.

corresponds to the vertex $\Gamma_{6}^{(0)}=Q_{6}$. All its solutions have the form

$$
\begin{aligned}
\frac{1}{n} H^{n} & =c_{30} \widetilde{\xi}+c_{31} & & \text { for } n \neq 0, \\
\ln H & =c_{30} \widetilde{\xi}+c_{31} & & \text { for } n=0,
\end{aligned}
$$

where $c_{30}$ and $c_{31}$ are arbitrary constants. These solutions do not go to infinity as $\widetilde{\xi} \rightarrow 0$.

The truncated equation (3.6) corresponds to the edge $\Gamma_{6}^{(1)}$. All its solutions have the form (3.7) and (3.8). They tend to the finite values $H=\left(c_{4} \ln \xi^{0}+c_{5}\right)^{1 / n}$ and $H=c_{4}\left(\xi^{0}\right)^{\lambda}$ as $\widetilde{\xi} \rightarrow 0$; that is, they do not go to infinity.

If $\xi^{0}+2 c_{2}=0$, then the vertex $Q_{3}$ disappears from the support of equation (5.2); but the cone for the problem only intersects the normal cones $\mathbf{U}_{6}^{(0)}$ and $\mathbf{U}_{6}^{(1)}$ as before, and the truncated equations corresponding to the faces $\Gamma_{6}^{(0)}$ and $\Gamma_{6}^{(1)}$ remain the same.

\section{Solutions of The BASIC EQUATIONS SATISFYING BOTH BOUNDARY CONDITIONS}

Here we study those solutions of equation (2.15) that satisfy conditions $(2.16),(2.17)$ and have the property (2.18). To do this we consider two cases separately: $c_{2} \geq 0$ and $c_{2}<0$.

\section{Case $c_{2} \geq 0$.}

Lemma 7. For fixed $c_{2} \geq 0$ and $n \in[0,1]$, equation (2.15) has a one-parameter family of solutions with the properties (2.16)-(2.18) and as $\xi \rightarrow 0$ the asymptotic behaviour is given by

$$
\begin{array}{ll}
H \sim \operatorname{const} \xi^{\lambda}, \quad \lambda<0, \quad \text { for } \quad n=0, \\
H \sim \text { const }|\ln \xi|^{1 / n} & \text { for } \quad n \neq 0 .
\end{array}
$$

Proof. According to Theorem 2, equation (2.15) has a one-parameter family of solutions (with respect to $c_{10}$ ) with the asymptotics (4.1). For $c_{10}<0$ these solutions are not 
TABLE 3. Values of $c_{3}$ on the family $\mathcal{M}_{1}$.

\begin{tabular}{|c|c|l|l|l|l|}
\hline$c_{2}$ & $n=0$ & $n=1 / 4$ & $n=1 / 2$ & $n=3 / 4$ & $n=1$ \\
\hline-1 & 2.05931 & 1.923155 & 1.847601 & 1.80526 & 1.78265 \\
-2 & 3.93106 & 3.87528 & 3.87168 & 3.89454 & 3.93164 \\
-3 & 6.21466 & 6.2403 & 6.30959 & 6.3998505 & 6.49607 \\
-4 & 8.78254 & 8.88927 & 9.03119 & 9.18598 & 9.34386 \\
-5 & 11.56874 & 11.755944 & 11.96998 & 12.19021 & 12.40807 \\
-6 & 14.53258 & 14.7996 & 15.08518 & 15.37037 & 15.6478 \\
-7 & 17.64631 & 17.9925 & 18.34908 & 18.69877 & 19.03536 \\
-8 & 20.8898 & 21.31453 & 21.74162 & 22.15536 & 22.55071 \\
-9 & 24.24772 & 24.755045 & 25.74162 & 25.72491 & 26.17866 \\
-10 & 27.70802 & 28.28825 & 28.8549 & 29.3955 & 29.90729 \\
\hline
\end{tabular}

constants and are decreasing as $\xi \rightarrow \infty$. According to Lemma 4, for $\xi>0$ they are decreasing monotonically to the value $H=1$. By Lemma 6 , these solutions do not come from infinity for any $\xi>0$. By Lemma 5 , none of these solutions tends to a finite value as $\xi \rightarrow 0$. Consequently, according to Theorem 1, all these solutions go to infinity with the asymptotics $(6.1)$ as $\xi \rightarrow 0$.

We introduce the families

$$
\mathcal{M}_{0}=\mathcal{M} \cap\left(\mathcal{G}_{1}^{(0)} \cup \mathcal{F}_{1}^{(1)} \mathcal{G}_{4}^{(0)} \cup \mathcal{F}_{1}^{(0)}\right) ; \quad \mathcal{M}_{1}=\mathcal{M} \cap \mathcal{F}_{1}^{(1)} \mathcal{G}_{4}^{(1)} .
$$

According to Theorems 1 and 2, they contain all the solutions of equation (2.15) that have the properties (2.16), (2.18) and tend to infinity as $\xi \rightarrow 0$. The family $\mathcal{M}_{1}$ exists only for $c_{2}<0$ and is the boundary of the family $\mathcal{M}_{0}$. Theorem 2 means that for $c_{2} \geq 0$, to the family $\mathcal{M}_{0}$ there corresponds the quadrant $\left\{c_{2} \geq 0, c_{10}<0\right\}$ of the $c_{2}, c_{10}$-plane.

Case $\boldsymbol{c}_{\mathbf{2}}<\mathbf{0}$. For $c_{2}<0$ the solutions with asymptotic behaviour (4.1) and with $c_{10}<0$ also increase monotonically as $\xi$ decreases from infinity; by Lemmas 4 and 7 they do not tend to infinity for finite $\xi$. But now, according to Lemma 5 , they may have a finite limit as $\xi \rightarrow 0$. We used two schemes of numerical calculations to analyse the solutions of equation $(2.15)$ for $c_{2}<0$.

Scheme 1. Write equation (2.15) in the form

$$
2\left[\frac{d H^{n}}{d t} \dot{H}\right] H-2 H^{n} \dot{H}^{2}+\left(e^{t}+2 c_{2}\right) \dot{H}=0,
$$

where $t=\ln \xi$ and $\stackrel{\text { def }}{=} d / d t$. Given the initial values $H_{0}$ and $\dot{H}_{0}$ for large negative values $t_{0}$, compute the solution by the Runge-Kutta method up to large positive values $t_{N}$.

Scheme 2. Take initial values of $H=1+y, H^{\prime}=y^{\prime}$ according to formulae (4.1), (4.2) for large $\xi=\xi_{0}$ near infinity. Here the infinite sum in formula (4.2) is replaced by an initial segment of it, and the values of the constant $c_{10}<0$ are taken in some grid in $\mathbb{R}$. The Runge-Kutta method is used to compute the solution of equation (2.15) up to some small $\xi_{N}>0$.

For the values

$$
n=0, \frac{1}{4}, \frac{1}{2}, \frac{3}{4}, 1
$$


TABLE 4 . Values of $c_{10}$ on the family $\mathcal{M}_{1}$.

\begin{tabular}{|c|l|l|l|l|l|}
\hline$c_{2}$ & $n=0$ & $n=1 / 4$ & $n=1 / 2$ & $n=3 / 4$ & $n=1$ \\
\hline-1 & -0.73231 & -0.80337 & -0.88023 & -0.96351 & -1.05338 \\
-2 & -0.41359 & -0.47024 & -0.53505 & -0.60844 & -0.69183 \\
-3 & -0.11085 & -0.12877 & -0.15048 & -0.17571 & -0.20536 \\
-4 & $-1.94 \cdot 10^{-2}$ & $-2.29 \cdot 10^{-2}$ & $-2.73 \cdot 10^{-2}$ & $-3.25 \cdot 10^{-2}$ & $-3.88 \cdot 10^{-2}$ \\
-5 & $-2.51 \cdot 10^{-3}$ & $-3.01 \cdot 10^{-3}$ & $-3.63 \cdot 10^{-3}$ & $-4.41 \cdot 10^{-3}$ & $-4.36 \cdot 10^{-3}$ \\
-6 & $-2.57 \cdot 10^{-4}$ & $-1.29 \cdot 10^{-4}$ & $-3.83 \cdot 10^{-4}$ & $-4.71 \cdot 10^{-4}$ & $-5.82 \cdot 10^{-4}$ \\
-7 & $-2.19 \cdot 10^{-5}$ & $-2.69 \cdot 10^{-5}$ & $-3.33 \cdot 10^{-5}$ & $-4.15 \cdot 10^{-5}$ & $-5.21 \cdot 10^{-5}$ \\
-8 & $-1.59 \cdot 10^{-6}$ & $-1.98 \cdot 10^{-6}$ & $-2.47 \cdot 10^{-6}$ & $-3.11 \cdot 10^{-6}$ & $-3.95 \cdot 10^{-6}$ \\
-9 & $-1.01 \cdot 10^{-7}$ & $-1.27 \cdot 10^{-7}$ & $-1.59 \cdot 10^{-7}$ & $-2.03 \cdot 10^{-7}$ & $-2.61 \cdot 10^{-7}$ \\
-10 & $-0.57 \cdot 10^{-8}$ & $-0.72 \cdot 10^{-8}$ & $-0.91 \cdot 10^{-8}$ & $-1.17 \cdot 10^{-8}$ & $-1.52 \cdot 10^{-8}$ \\
\hline
\end{tabular}

TABLe 5. The minima of $c_{10}$ on the family $\mathcal{M}_{1}$.

\begin{tabular}{|c|c|l|l|}
\hline$n$ & $c_{2}$ & \multicolumn{1}{|c|}{$c_{3}$} & $c_{10}$ \\
\hline 0 & -1.034 & 2.11366 & -0.733023 \\
$1 / 4$ & -1.059 & 2.0226 & -0.805886 \\
$1 / 2$ & -1.088 & 2.003022 & -0.88612 \\
$3 / 4$ & -1.117 & 2.02054 & -0.974637 \\
1 & -1.15 & 2.06913 & -1.07229 \\
\hline
\end{tabular}

and for fixed values of $c_{2}<0$, first we calculated the solutions in the family $\mathcal{M}_{1}$. For scheme 1 the initial data were chosen according to formula (3.4) and the constant $c_{3}$ was varied so that the solution tended to $H=1$ as $t \rightarrow+\infty$. For the resulting solution, the value of the constant $c_{10}$ was found by the first formula (4.1). Then, for control, the solution was computed by the second scheme with these values of the constants $c_{2}$ and $c_{10}$, and the corresponding initial data of the first scheme were obtained. The results of these calculations with respect to the grid $c_{2}=-1(-1)-10$ are presented in Tables 3 and 4. Figure 12 shows the graphs of the functions $c_{3}=æ_{3}\left(c_{2}\right)$ and $c_{10}=æ_{10}\left(c_{2}\right)$ for the values (6.4). It turns out that on the family $\mathcal{M}_{1}$ the values of the constant $c_{3}$ increase monotonically as $c_{2}$ decreases, and the values of the constant $c_{10}$ have a minimum. Table 5 contains the values of $c_{2}, c_{3}, c_{10}$ at the minimum points of $c_{10}$ on the family $\mathcal{M}_{1}$. Figure 13 shows the graphs of the solutions $H(\xi)$ and $G(\xi)$ of the system (2.12), (2.10) for $n=0$ and $n=1$ for the values of $c_{2}, c_{3}$, and $c_{10}$ corresponding to the minima of $c_{10}$, that is, as in Table 5 .

Since the family $\mathcal{M}_{1}$ is the boundary of the family $\mathcal{M}_{0}$, for the values of the constant $c_{10}$ that are smaller than its values on the family $\mathcal{M}_{1}$, the solution belongs to the family $\mathcal{M}_{0}$ and has the asymptotics (3.7), (3.8) as $\xi \rightarrow 0$. Everything is clear about the asymptotic behaviour in (3.7), but for the asymptotic behaviour in (3.8) it would be of interest to know the values of the exponent $\lambda$. For $n=0$ for various values of $c_{2}$ and $c_{10}<0$ corresponding to the family $\mathcal{M}_{0}$, the solutions were computed by the second scheme and the limit of the ratio $\ln H / \ln \xi=\lambda$ as $\xi \rightarrow 0$ was computed. The results are presented 

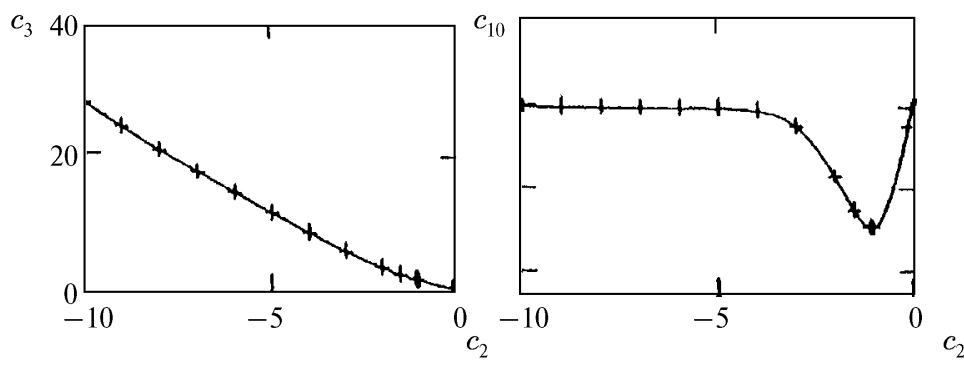

$n=0$

$c_{3} 40$
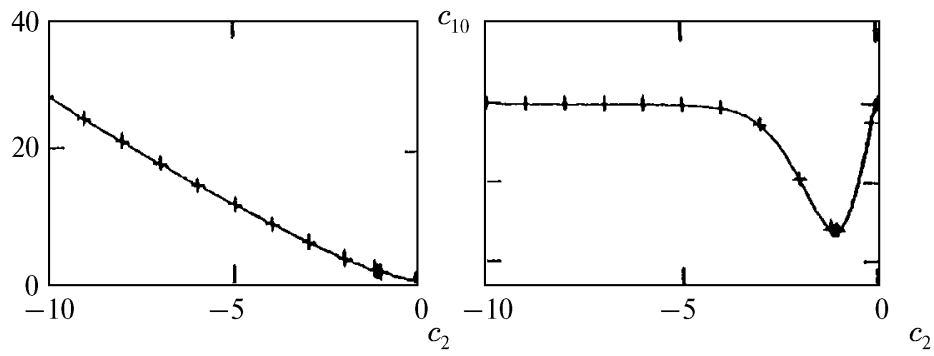

$n=\frac{1}{4}$

$c_{3}$

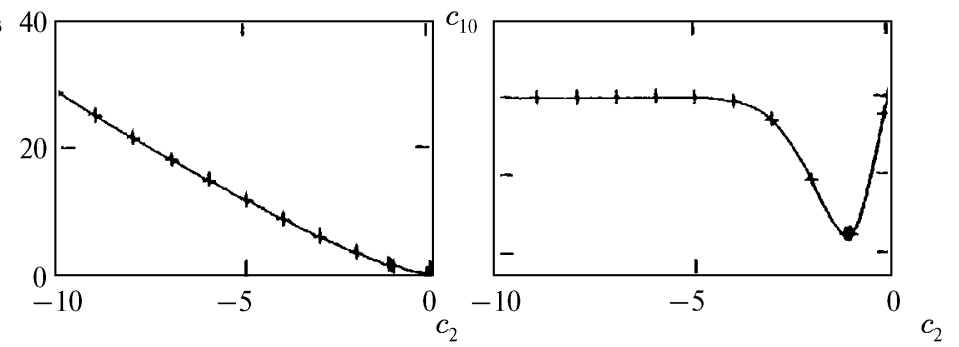

$$
n=\frac{1}{2}
$$
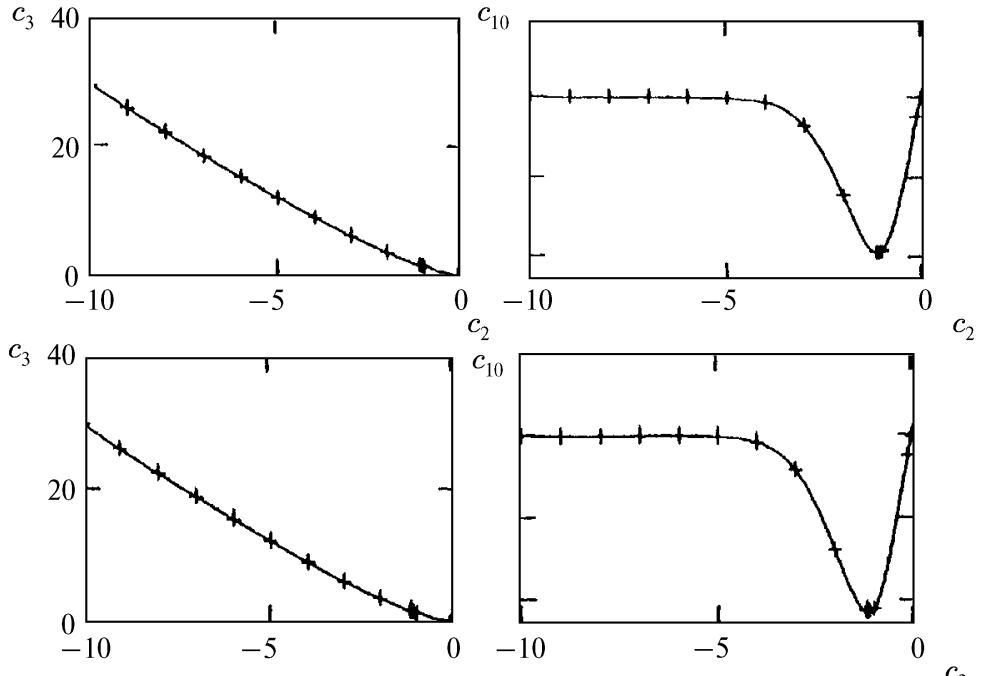

$$
n=\frac{3}{4}
$$$$
n=1
$$

FiguRE 12. The graphs of the functions $c_{3}=æ_{3}\left(c_{2}\right)$ and $c_{10}=æ_{10}\left(c_{2}\right)$ for the family $\mathcal{M}_{1}$ and the values (6.4).

in Table 6. Figure 14 shows the graphs of the solution $H(\xi)$ for $n=0$ and $1, \quad c_{2}=0$ and $10, c_{10}=-1$. 
TABLE 6 . The values of $-\lambda$ for $n=0$ on the family $\mathcal{M}_{0}$.

\begin{tabular}{|c|c|c|c|c|c|c|c|c|c|c|c|}
\hline$c_{10} c_{2}$ & -5 & -4 & -3 & -2 & -1 & 0 & 1 & 2 & 3 & 4 & 5 \\
\hline-1 & 11.4 & 7.22 & 3.69 & 1.23 & 0.337 & 0.591 & 1.35 & 2.27 & 3.24 & 4.23 & 5.23 \\
\hline-2 & 11.6 & 8.33 & 4.77 & 2.15 & 0.894 & 0.899 & 1.54 & 2.41 & 3.35 & 4.33 & 5.31 \\
\hline-3 & 12.2 & 8.98 & 5.38 & 2.69 & 1.27 & 1.12 & 1.67 & 2.50 & 3.43 & 4.39 & 5.37 \\
\hline-4 & 13.7 & 9.43 & 5.82 & 3.08 & 1.56 & 1.28 & 1.77 & 2.57 & 3.48 & 4.43 & 5.40 \\
\hline-6 & 14.3 & 10.1 & 6.43 & 3.63 & 1.97 & 1.54 & 1.92 & 2.67 & 3.56 & 4.50 & 5.46 \\
\hline-8 & 14.8 & 10.5 & 6.86 & 4.02 & 2.27 & 1.73 & 2.04 & 2.75 & 3.62 & 4.55 & 5.51 \\
\hline-10 & 15.1 & 10.9 & 7.19 & 4.31 & 2.51 & 1.89 & 2.14 & 2.82 & 3.67 & 4.59 & 5.54 \\
\hline
\end{tabular}

Thus, for fixed $n \in[0,1]$ on the family $\mathcal{M}_{1}$ the way the constants $c_{3}$ in (3.4) and $c_{10}$ in (4.1) depend on $c_{2}<0$ are given by the curves

$$
c_{3}=æ_{3}\left(c_{2}, n\right) \quad \text { and } \quad c_{10}=æ_{10}\left(c_{2}, n\right) .
$$
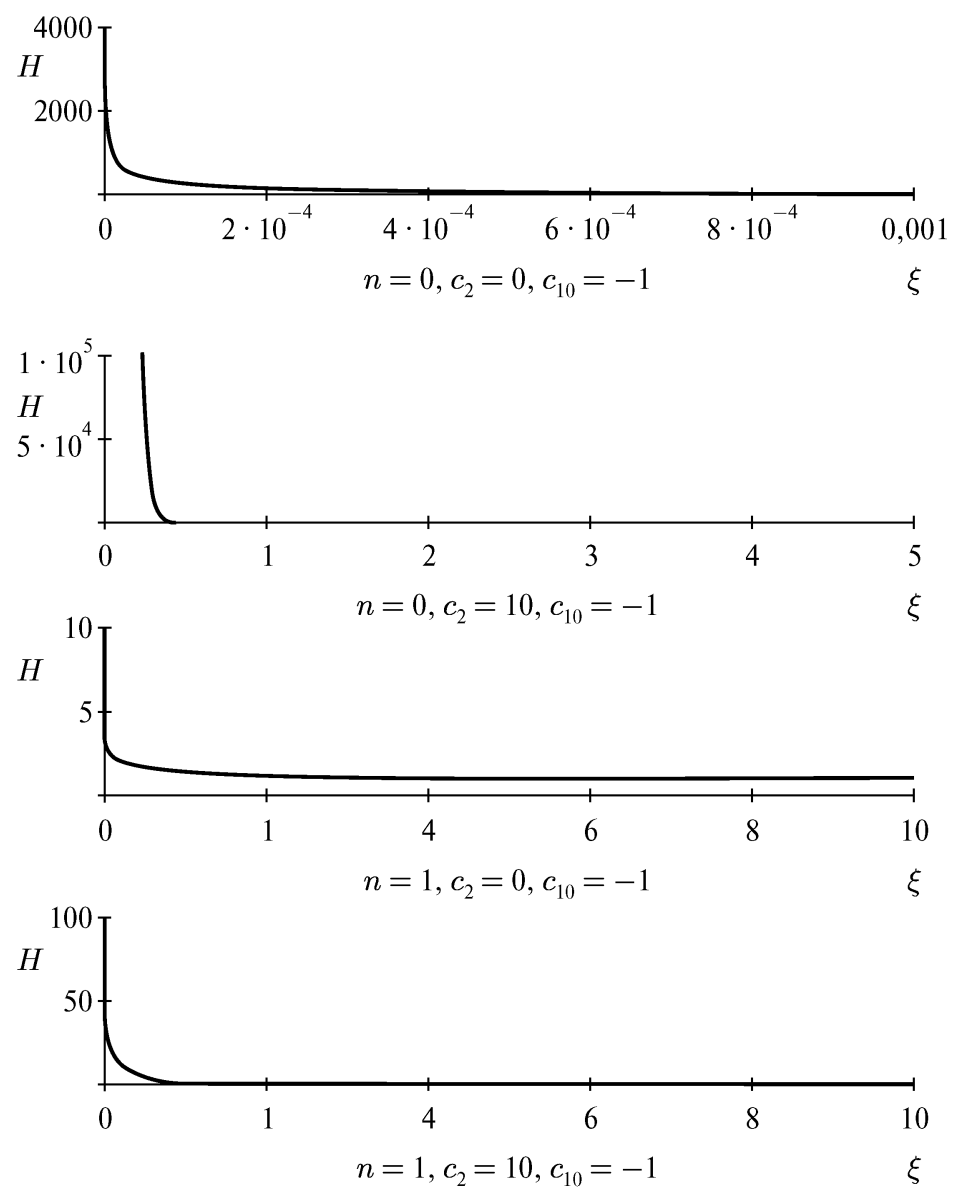

Figure 13. The graphs of the functions $H(\xi)$ and $G(\xi)$ for the solution of the system (2.12), (2.10) for $n=0$ and $n=1$ with the minimal value of $c_{10}=æ_{10}\left(c_{2}\right)$. 

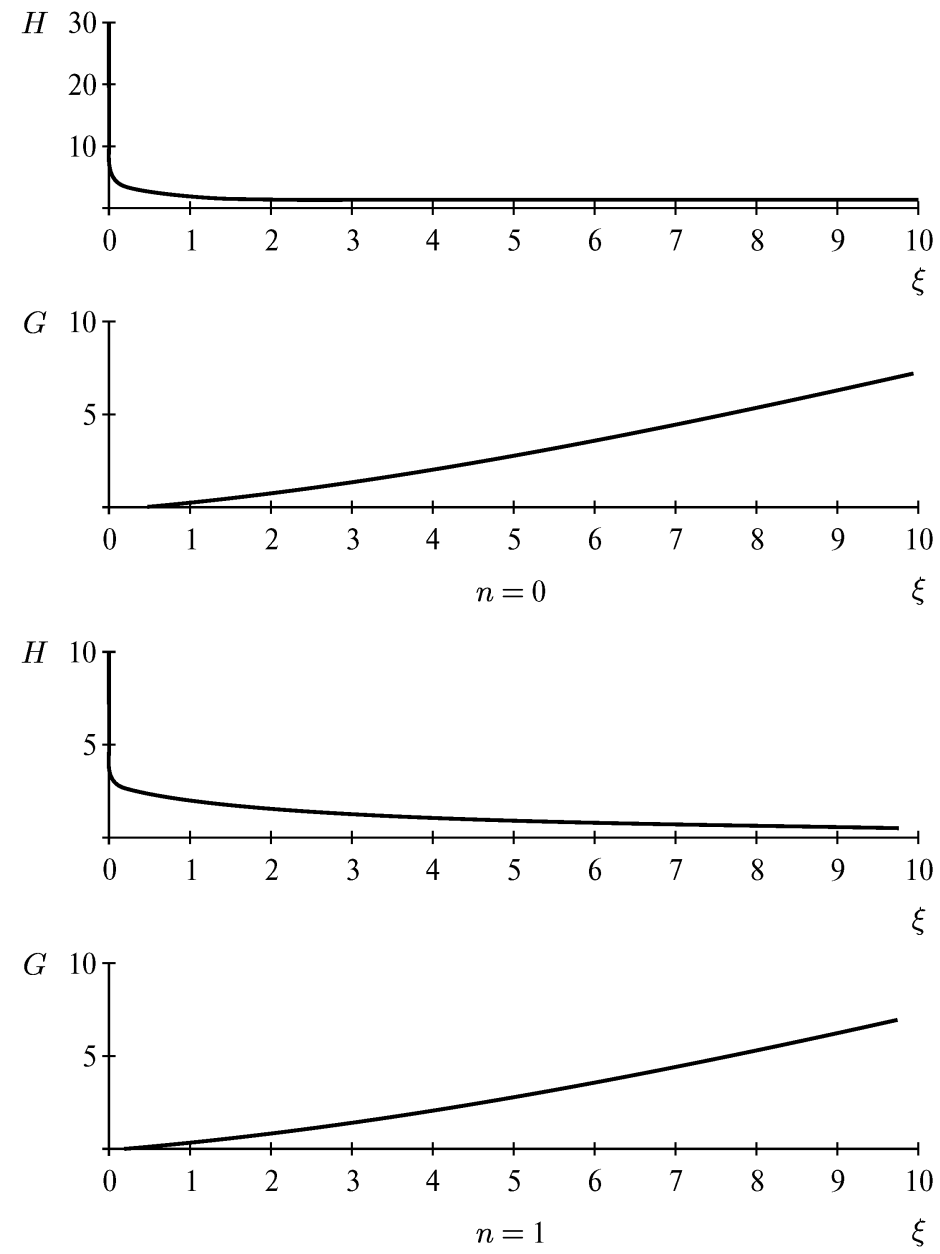

FiguRE 14. The graphs of the function $H(\xi)$ for the solutions of the system (2.12), (2.10) for $n=0,1 ; c_{2}=0,10 ; c_{10}=-1$.

Thus, we have obtained the following.

Lemma 8. For fixed $n \in[0,1]$ the family $\mathcal{M}_{0}$ contains all those solutions in the family $\mathcal{M}$ for which $c_{10}<æ_{10}\left(c_{2}\right)$.

Combining Lemmas 7 and 8 results in the following.

Theorem 3. For fixed $n \in[0,1]$ the family $\mathcal{M}_{0}$ contains all those solutions in the family $\mathcal{M}$ for which

$$
c_{10}<\left\{\begin{array}{lll}
æ_{10}\left(c_{2}\right) & \text { if } \quad c_{2}<0 \\
0 & \text { if } \quad c_{2} \geq 0 .
\end{array}\right.
$$

These solutions have the asymptotics (6.1) as $\xi \rightarrow 0$. 


\section{Return to the original PROBlem}

If we subject the solutions $H(\xi)$ of equation (2.15) to the inverse transformations of those indicated in Lemma 3, then we obtain solutions of the truncated system (1.16)(1.18). Then corresponding to the families $\mathcal{M}_{0}$ and $\mathcal{M}_{1}$ of solutions of the problem (2.15)-(2.17) there will be families $\widetilde{\mathcal{M}}_{0}$ and $\widetilde{\mathcal{M}}_{1}$ of self-similar solutions of the problem (1.13), (1.14), (1.16)-(1.18). Namely, first we obtain $G(\xi)$ in the problem (2.4), (2.10)-(2.12), and we have $G \sim \xi / H$ as $\xi \rightarrow 0$, that is,

$$
\begin{aligned}
& G \sim \operatorname{const} \xi^{1-\lambda}, \quad \lambda<0, \quad \text { for } \quad n=0, \\
& G \sim \frac{\operatorname{const} \xi}{|\ln \xi|^{1 / n}} \quad \text { for } n \neq 0 \text {. }
\end{aligned}
$$

Then, after the making the inverse transformation to $(2.8)$, we obtain the solutions $G(\xi)$ and $H(\xi)$ of the problem (2.2)-(2.4). As $\xi \rightarrow 0$ they have the asymptotics (7.1) and

$$
\begin{array}{ll}
H \sim \operatorname{const} \xi^{\lambda}, \quad \lambda<0, & \text { for } \quad n=0, \\
H \sim \operatorname{const}|\ln \xi|^{1 / n} & \text { for } \quad n \neq 0 .
\end{array}
$$

From (2.1) we obtain $\mathrm{P}(\xi)$ and as $\xi \rightarrow 0$ it has the asymptotic behaviour

$$
\begin{aligned}
& \mathrm{P} \sim \operatorname{const} \xi^{-\lambda}, \quad \lambda<0, \quad \text { for } n=0, \\
& \mathrm{P} \sim \frac{\text { const }}{|\ln \xi|^{1 / n}} \quad \text { for } n \neq 0 .
\end{aligned}
$$

On passing from the self-similar coordinates (1.19) to the original ones, we obtain the solutions $\psi, \rho, h$ of the problem $(1.13),(1.14),(1.16)-(1.18)$. In the boundary layer as $\xi \rightarrow 0$ they have the asymptotics

$$
\begin{array}{lll}
\psi \sim \operatorname{const} x \xi^{1-\lambda}, & \rho \sim \operatorname{const} \xi^{-\lambda}, & h \sim \operatorname{const} \xi^{\lambda}, \quad \lambda<0, \quad n=0 ; \\
\psi \sim \frac{\text { const } r^{2}}{|\ln \xi|^{1 / n}}, & \rho \sim \frac{\mathrm{const}}{|\ln \xi|^{1 / n}}, & h \sim \operatorname{const}|\ln \xi|^{1 / n}, \quad n \in(0,1] .
\end{array}
$$

Thus, we have obtained the solutions and their asymptotics for the family $\widetilde{\mathcal{M}}_{0}$. In a similar fashion we obtain the solutions for the family $\widetilde{\mathcal{M}}_{1}$, which as $\xi \rightarrow 0$ have the asymptotics

$$
\psi \sim \frac{\text { const } r^{2}}{|\ln \xi|^{1 /(n+1)}}, \quad \rho \sim \frac{\text { const }}{|\ln \xi|^{1 /(n+1)}}, \quad h \sim \text { const }|\ln \xi|^{1 /(n+1)}, \quad n \in[0,1] .
$$

By Theorem 1 of Chapter I the solutions in the families $\widetilde{\mathcal{M}}_{0}$ and $\widetilde{\mathcal{M}}_{1}$ are asymptotics of solutions of the problem (1.7)-(1.9), (1.12)-(1.13) for $x \rightarrow+\infty$ and $r^{2} / x<\infty$ if such solutions exist.

Thus, we have obtained the following main result.

Theorem 4. In the boundary layer $r^{2} / x<\infty$ as $x \rightarrow+\infty$ the problem (1.7)-(1.9), (1.12)-(1.13) has families of solutions $\widetilde{\widetilde{\mathcal{M}}}_{0}$ and $\widetilde{\widetilde{\mathcal{M}}}_{1}$. Near the needle, as $\xi=r^{2} / x \rightarrow 0$, $\widetilde{\widetilde{\mathcal{M}}}_{0}$ has the asymptotics (7.2) and $\widetilde{\widetilde{\mathcal{M}}}_{1}$ has (7.3). When the parameters of the problem (1.7)-(1.9), (1.12)-(1.13) are fixed, the family $\widetilde{\widetilde{\mathcal{M}}}_{0}$ is a two-parameter family, while $\widetilde{\widetilde{\mathcal{M}}}_{1}$ is a one-parameter family and is the boundary of the family $\widetilde{\widetilde{\mathcal{M}}}_{0}$.

In the boundary layer $\xi \in(0, \infty)$, the families $\widetilde{\mathcal{M}}_{0}$ and $\widetilde{\mathcal{M}}_{1}$ of self-similar solutions of the truncated system $(1.16)-(1.18)$ are asymptotics of solutions in the families $\widetilde{\widetilde{\mathcal{M}}}_{0}$ and 
$\widetilde{\widetilde{\mathcal{M}}}_{1}$ of the original problem (1.7)-(1.9), (1.12)-(1.13). The accuracy of the asymptotics can be estimated by using Remark 1 of Chapter I.

Theorem 5. As $x \rightarrow+\infty$ the accuracy of the asymptotics in the families $\widetilde{\mathcal{M}}_{0}$ and $\widetilde{\mathcal{M}}_{1}$ in the boundary layer $\xi \in(0, \infty)$ is estimated by the formulae

$$
\psi-\widetilde{\psi}=\widetilde{\psi} O\left(x^{-\varepsilon}\right), \quad \rho-\widetilde{\rho}=\widetilde{\rho} O\left(x^{-\varepsilon}\right), \quad h-\widetilde{h}=\widetilde{h} O\left(x^{-\varepsilon}\right),
$$

where $0<\varepsilon<1$ and $\widetilde{\psi}, \widetilde{\rho}, \widetilde{h}$ are asymptotics in the families $\widetilde{\mathcal{M}}_{0}$ and $\widetilde{\mathcal{M}}_{1}$, while $\psi, \rho, h$ are solutions in the families $\widetilde{\widetilde{\mathcal{M}}}_{0}$ and $\widetilde{\widetilde{\mathcal{M}}}_{1}$.

Proof. By Lemma 2, the vector $P=(2,1,2,0,0)$ is a normal vector of the truncated system (1.16)-(1.18). To apply Remark 1 of Chapter I we need $p_{1}=1$. Therefore we must use the vector $P^{*}=P / 2=(1,1 / 2,1,0,0)$. Then in Remark 1 of Chapter I we have

$$
\begin{aligned}
& \alpha_{1}=\left\langle P^{*}, Q_{2}\right\rangle=-\frac{1}{2}, \\
& \beta_{1}=\left\langle P^{*}, Q_{1}\right\rangle=\left\langle P^{*}, Q_{3}\right\rangle=-\frac{3}{2}, \\
& \alpha_{2}=\left\langle P^{*}, Q_{5}\right\rangle=\left\langle P^{*}, Q_{6}\right\rangle=\left\langle P^{*}, Q_{8}\right\rangle=-1, \\
& \beta_{2}=\left\langle P^{*}, Q_{7}\right\rangle=-2, \\
& \alpha_{3}=\left\langle P^{*}, Q_{9}\right\rangle=\left\langle P^{*}, Q_{12}\right\rangle=\left\langle P^{*}, Q_{13}\right\rangle=-1, \\
& \beta_{3}=\left\langle P^{*}, Q_{10}\right\rangle=\left\langle P^{*}, Q_{14}\right\rangle=-2 .
\end{aligned}
$$

Consequently, in formula (1.6) of Chapter I we have

$$
0<\varepsilon<\varepsilon_{0}=\min _{1 \leq i \leq 3}\left|\alpha_{i}-\beta_{i}\right|=1,
$$

that is, we have obtained the estimate (7.4).

\section{Conclusion}

To the authors' surprise, the negative result in Chapter II raised doubts among specialists in hydromechanics.

In particular, Kulikovskil expressed the following viewpoint. In the problem of a viscous incompressible flow in an infinite cylinder, a particular solution is known, which is called the Poiseuille flow. A flow is also known when a smaller cylinder is placed coaxially inside a larger cylinder. In this case the solution is practically the same as the Poiseuille flow, except for a small layer near the inner cylinder. Here, the adhesion conditions hold at both cylinders. If the radius of the inner cylinder tends to zero (that is, a needle is obtained in the limit), then in the limit the same Poiseuille flow in the large cylinder is obtained, which ignores the needle situated on the axis of the cylinder. This point seems to be convincing to other specialists in mechanics.

We can reply to this consideration that the flow thus obtained does not satisfy the adhesion conditions at the needle and, consequently, is not a solution of the problem of a flow past a needle.

The following simple example suggested by Bakhvalov may serve to confirm the result about the non-existence of solutions in the problem of a flow of a viscous incompressible fluid past a (semi-)infinite needle. Consider the "crosssection" through the cylinders, that is, two concentric circles on the plane. If proper boundary conditions for the Laplace operator on the plane are given at these circles, then this Dirichlet problem in the annulus between the circles always has a unique solution. If the radius of the inner circle tends to zero, then in the limit we obtain a Dirichlet problem in a disc with a punctured point with boundary conditions at the outer circle and at the punctured point. As a 
rule, such a Dirichlet problem has no solutions, since the Dirichlet problem in the disc without puncture has a unique solution. If the value of this solution at the punctured point is different from the given value, then the problem has no solutions. The same example was given in the referee's report on the paper [15. Furthermore, the referee and independently Kondrat'ev agree with the negative result of Chapter II.

In the authors' opinion, the non-existence of a flow can be explained from the physical viewpoint as follows. The adhesion condition at the needle is stronger than at a plate. Therefore, for a non-steady flow of a viscous incompressible fluid past a semi-infinite needle, the fluid will stick to the needle; this clot (that is, a volume with very low flow-speed) will enlarge, fill the entire space and stop the flow.

In any case, nobody has produced a solution satisfying all the boundary conditions, even with an arbitrarily complicated singularity.

Birman noted that to check whether it is possible for the adhesion condition on the needle to be satisfied can be done using the linear approximation of the Navier-Stokes equations, that is, by the Stokes equations or, more precisely, by their truncated system. Indeed, for the case of incompressible fluid, if in the system (1.1) of Chapter II the nonlinear terms are discarded and for the resulting system the truncated system is selected corresponding to the upper boundary conditions in (2.1), then we obtain the system

$$
\frac{1}{\rho} \frac{\partial p}{\partial x}=\nu\left(\frac{\partial^{2} u}{\partial y^{2}}+\frac{\partial^{2} u}{\partial z^{2}}\right), \quad \frac{1}{\rho} \frac{\partial p}{\partial y}=\frac{1}{\rho} \frac{\partial p}{\partial z}=0 .
$$

Its self-similar solutions have $p=$ const, that is, we obtain the single equation

$$
\frac{\partial^{2} u}{\partial y^{2}}+\frac{\partial^{2} u}{\partial z^{2}}=0
$$

with the boundary conditions $u=u_{\infty} \neq 0$ as $y^{2}+z^{2} \rightarrow \infty$, and $u=0$ for $y=z=0$. As mentioned above, this problem has no solutions. Hence for a fluid the adhesion conditions at the needle cannot be satisfied even for a three-dimensional flow that is not axisymmetric. Consequently, for the fluid, increasing the number of dependent variables at the expense of abandoning the axial symmetry of the flow does not allow one to obtain a solution.

For the case of a gas, corresponding to the truncation of the linear part of the original system (1.1)-(1.3) of Chapter III we have the part of the truncated system (1.16)-(1.18) in Chapter III that is linear in $\psi$. After introducing the self-similar coordinates (1.19) from Chapter III, the truncated system reduces to the system of ODE (2.2) in Chapter III. Its part that is linear in $G$ consists of the equation

$$
2 C^{n}\left[H^{n}\left(G^{\prime} H\right)^{\prime} \xi\right]^{\prime}=0
$$

and equation (2.7). For this system, equation (2.5) of Chapter III distinguishes an invariant manifold, on which this system reduces to equation (2.15) of Chapter III. The analysis of the solutions of this equation carried out in $\S 3$ of Chapter III shows that here the adhesion conditions can be satisfied.

Aksenov considers that the reason for the negative result for the fluid is the fact that near the needle of very small diameter, comparable with the size of molecules of the fluid, the Navier-Stokes equations are inapplicable. He adduces the following argument. For the Poiseuille flow between two cylinders, mentioned above, when the inner cylinder has a very small diameter, the friction at it is of the same order as at the outer cylinder. This contradicts mechanical intuition. 


\section{REFERENCES}

[1] L. Prandtl, Über Flüssigkeitsbewegung bei sehr kleiner Reibung, Verhandlungen des III. Internat. Math.-Kongr., Heidelberg, 1904, Teubner, Leipzig, 1905, S. 484-491.

[2] H. Blasius, Grenzschichten in Flüssigkeiten mit kleiner Reibung, Z. Math. Phys. 56 (1908), 1-37.

[3] S. Goldstein, On the two-dimensional steady flow of a viscous fluid behind a solid body. I, II, Proc. Royal Soc. London A 142 (1933), 545-573.

[4] K. Stewartson, On asymptotic expansions in the theory of boundary layers, J. Math. Phys. 36 (1957), 173-191. MR0093232(19:1219h)

[5] A. I. van de Vooren and D. Dijkstra, The Navier-Stokes solution for laminar flow past a semi-infinite flat plate, J. Engineer. Math. 4 (1970), 9-27.

[6] R. I. MacLachlan, The boundary layer on a finite flat plate, Phys. Fluids A 3 (1991), 341-348.

[7] R. A. Seban and R. Bond, Skin-friction and heat-transfer characteristics of a laminar boundary layer on a cylinder in axial incompressible flow, J. Aeronaut. Sci. 18 (1951), 671-675.

[8] H. R. Kelly, A note on the laminar boundary layer on a circular cylinder in axial incompressible flow, J. Aeronaut. Sci. 21 (1954), 634.

[9] Lord Rayleigh, On the motion of solid bodies through viscous liquid, Phil. Mag. (6) 21 (1911), $697-711$.

[10] K. Pohlhausen, Zur näherungsweisen Integration der Differentialgleichung der laminaren Grenzschicht, Z. Angew. Math. Mech. 1 (1921), 252-268.

[11] M. B. Glauert and M. J. Lighthill, The axisymmetric boundary layer on a long thin cylinder, Proc. Roy. Soc. London A 230 (1955), 188-203. MR0070373 (16:1171g)

[12] K. Stewartson, The asymptotic boundary layer on a circular cylinder in axial incompressible flow, Quart. Appl. Math. 13 (1955), 113-122. MR0068954(16:968c)

[13] A. D. Bryuno, Power geometry in algebraic and differential equations, Nauka, Fizmatlit, Moscow, 1998; English transl., North-Holland, Amsterdam, 2000. MR1734769 (2001d:37065)

[14] A. D. Bryuno and T. V. Shadrina, On an axially symmetric flow of a viscous incompressible fluid around a needle, Dokl. Akad. Nauk 387 (2002), 589-595; English transl. in Dokl. Math. 66 (2002), 396-402. MR2004520 (2004f:76046)

[15] T. V. Shadrina, The axially symmetric boundary layer around a needle, Proc. BAIL 2002 (Eds. S. Wang and N. Fowkes), Univ. of Western Australia, Perth, 2002, pp. 213-220.

[16] A. D. Bryuno and T. V. Shadrina, An axisymmetric boundary layer on a needle, Dokl. Akad. Nauk 394 (2004), 298-304; English transl. in Dokl. Math. 69 (2004), 57-63. MR2088482 (2005g:76105)

[17] T. V. Shadrina, Boundary layer for axisymmetric flow past a needle, Differential Equations 38 (2002), 853-854. (Russian)

[18] T. V. Shadrina, On axisymmetric flow of a viscous incompressible fluid past a needle, XXVIII Gagarin lectures, Absracts, vol. 2, MATI, Moscow, 2002, 98-99. (Russian)

[19] A. D. Bryuno and T. V. Shadrina, The axially symmetric boundary layer around a needle, Intern. Conf. on Differential and Functional Differential Equations, MAI, Moscow, 2002, pp. 18-19.

[20] A. D. Bryuno and T. V. Shadrina, The axially symmetric boundary layer around a needle, Intern. Conf. "Navier-Stokes Equations and Related Topics (NSEC8)", Abstracts, Euler Inst., S. Petersburg, 2002, pp. 18-19.

[21] T. V. Shadrina, Axisymmetric boundary layer on a needle, XIV All-Russia Conf. "Theoretical Foundations and Construction of Algorithms for Solving Problems of Mathematical Physics", Abstracts, Abrau-Dyurso, 2002, pp. 167-168. (Russian)

[22] A. D. Bryuno and T. V. Shadrina, The axially symmetric boundary layer on a needle, Intern. Conf. on Boundary and Interior Layers, ONERA, Toulouse, 2004, July 5th, 11.20, pp. 1-10.

[23] A. D. Bryuno and T. V. Shadrina, Boundary layer on a needle, II All-Russia Conf. "Topical Problems of Applied Mathematics and Mechanics", Abrau-Dyurso, 2004, pp. 30-32. (Russian)

[24] T. V. Shadrina, On axisymmetric flow of a viscous incompressible fluid past a needle, Preprint no. 36, Keldysh Inst. Appl. Math., Moscow, 2002. (Russian)

[25] A. D. Bryuno and T. V. Shadrina, Axisymmetric boundary layer on a needle, Preprint no. 64, Keldysh Inst. Appl. Math., Moscow, 2003. (Russian)

[26] A. D. Bryuno and T. V. Shadrina, Methods of investigation of a boundary layer on a needle, Preprint no. 35, Keldysh Inst. Appl. Math., Moscow, 2004. (Russian)

[27] A. D. Bryuno and T. V. Shadrina, On incompressible boundary layer on a needle, Preprint no. 36, Keldysh Inst. Appl. Math., Moscow, 2004. (Russian)

[28] A. D. Bryuno and T. V. Shadrina, Compressible heat-conducting boundary layer on a needle, Preprint no. 37, Keldysh Inst. Appl. Math., Moscow, 2004. (Russian)

[29] A. D. Bryuno, Self-similar solutions and power geometry, Uspekhi Mat. Nauk 55 (2000), no. 1, 3-44; English transl. in Russian Math. Surveys 55 (2000), no. 1, 1-42. MR1751817 (2001g:34007) 
[30] A. D. Bryuno, Power expansions of solutions of a system of algebraic and differential equations, Dokl. Akad. Nauk 380 (2001), 298-304; English transl. in Dokl. Math. 64 (2001), 180-186. MR.1868120(2002h:34672)

[31] A. D. Bryuno, Power asymptotics of solutions to an ordinary differential equation, Dokl. Akad. Nauk 392 (2003), 295-300; English transl. in Dokl. Math. 68 (2003), 199-203. MR2088468 (2005g:34120)

[32] A. D. Bryuno, Asymptotics and expansions of solutions of an ordinary differential equation, Preprint no. 9, Keldysh Inst. Appl. Math., Moscow, 2003. (Russian)

[33] A. D. Bryuno, Asymptotic behavior and expansions of solutions of an ordinary differential equation, Uspekhi Mat. Nauk 59 (2004), no. 3, 31-80; English transl. in Russian Math. Surveys 59 (2004), 429-480. MR:2116535 (2005m:34008)

[34] A. D. Bryuno, Power expansions of solutions of an algebraic or a differential equation, Dokl. Akad. Nauk 380 (2001), 155-159; English transl. in Dokl. Math. 64 (2001), 160-164. MR1873273 (2002j:34006)

[35] A. D. Bryuno, Power-logarithmic expansions of solutions of an ordinary differential equation, Dokl. Akad. Nauk 392 (2003), 439-444; English transl. in Dokl. Math. 68 (2003), 221-226. MR2081499

[36] A. D. Bryuno, Nonpower asymptotics of solutions of an ordinary differential equation, Dokl. Akad. Nauk 392 (2003), 586-591; English transl. in Dokl. Math. 68 (2003), 242-246. MR2082848 (2005e:34155)

[37] L. G. Loutsyanskiı̌, Mechanics of liquids and gases, Nauka, Moscow, 1978; English transl. of 2nd ed., Pergamon Press, Oxford, 1972. MR0197015 (33:5199)

[38] G. K. Batchelor, An introduction to fluid dynamics, Cambridge Univ. Press, Cambridge, 1999. MR 1744638 (2000j:76001)

[39] L. G. Loŭtsyanskiǔ, Laminar boundary layer, Nauka, Fizmatlit, Moscow, 1962.

[40] É. Kamke, Differentialgleichungen. Lösungsmethoden und Lösungen. I: Gewöhnliche Differentialgleichungen, B. G. Teubner, Stuttgart, 1977. MR0466672 (57:6549)

[41] M. M. Vasil'ev, On axisymmetric flows of a viscous heat-conducting gas, Preprint no. 11, Keldysh Inst. Appl. Math., Moscow, 2001. (Russian)

[42] M. M. Vasil'ev, Obtaining the self-similar asymptotics of solutions to the Navier-Stokes equations by power geometry, Progress in Analysis, Proc. 3rd Intern. ISAAC Congr. (Eds. H. G. Begehr, R. P. Gilbert, and M. W. Wong), vol. 1. World Sci., River Edge, NJ, 2003, pp. 93-101. MR2032673

[43] A. D. Bryuno, Complicated expansions of solutions of an ordinary differential equation, Preprint no. 36, Keldysh Inst. Appl. Math., Moscow, 2005. (Russian)

Keldysh Institute of Applied Mathematics, Miusskaya Pl. 4, Moscow 125047, Russia

E-mail address: bruno@keldysh.ru

Keldysh Institute of Applied Mathematics, Miusskaya Pl. 4, Moscow 125047, Russia

E-mail address: shadrina@keldysh.ru 